\title{
Interprocess Communication for Distributed Robotics
}

\author{
David Gauthier \\ Bachetor of Engineering (McGill University). 1983 \\ Bactielor of Science (MicGill University) 1978
}

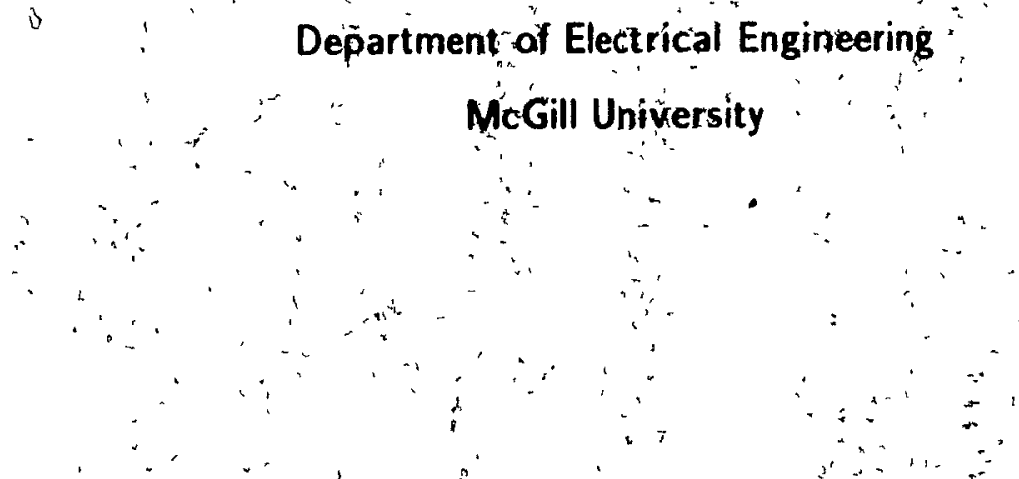

A thesis submitted to the Faculty of Graduate Studies and Research in partial fulfillment of the requirements for the degree of.

Mastèr of Engineering.

July 1986

(C) David Gauthier 
Date

MOY DE L'AUTEUR:

DÉPARTEAENT:

GRADE:

TITRE DE LA TAÉSE:

4

1. Par la presente, I'auteur accorde a l'universite McGill I'qutorisation dé mettre cette thèse li disposition dés lecteurs dans une blblothęque de McGili ou une autre blbllótheque, solt sous sa forme actuelfe, solt soug forme d'une. rẹptoduction. L'aúteur dEtient cependánt les autres drofts de publications il est éntendu, par allieurs, que ni. Ia thêse, nt les longs extratts de cette thèse ne pourront être, Imprimés: ou reprodults par' d'autres moyens Bans ' 1 'autorigation Ecrite, de 1 'auteur.

2. La presente autorisation entré en vigueur a la date Indiquée c1-dèssús ấmoting que le Cónt exécut1f du cónsell n'alt voté dê differer cette date. Dans ce cas la date differé sera lie

81 oneture du doyen el une dafe figure a l'alinea 2 :

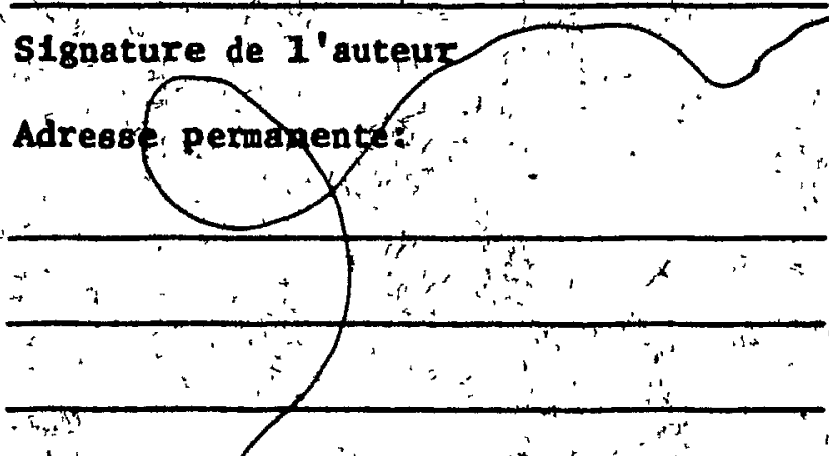




\section{Résumé}

Les applications robotiques ávancées exigent de la part des robots un comporte-

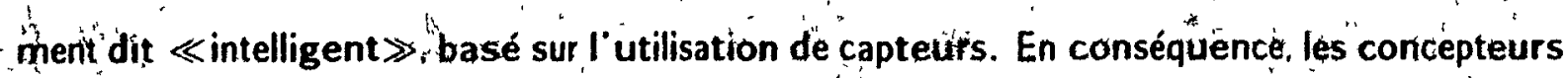
de têls systèmes doivent faire face à des systèmes comportant un nombre croissant dé prócesseurs pour la commande des robots et le traitement des données. Il est maintenant commode d'utiliser des réseaux, locaux pour standardiser l'intercoñnection physiqué de ces éléments et les protocöles de communiçation.

Le laboratoire de Visionique et de Robotique (Computer Vision and Robotics Laboratóry), au Département de Génie Electrique de MçGill University possède un système informatique distrịbué. basé sur un réseau local Ethernet reliant des ordinateuŕs opérant sous lesystème dexploitation UNIX 4:2BSD comportant plusieurs robots. des systênes

de visionique et autres capteurs sensoriels. La programmation d un système distribué té qué celui déctrit requiert que les processus aient aćcès des moyens de communication sûrs. efficacèśst d'utilișation cỏmmádé,

"Cette thèse décrit la conception. larchitecture la mise en ceuvie et lanalyse des performances d'un environnement de programmation distribuée pouvant servir de base

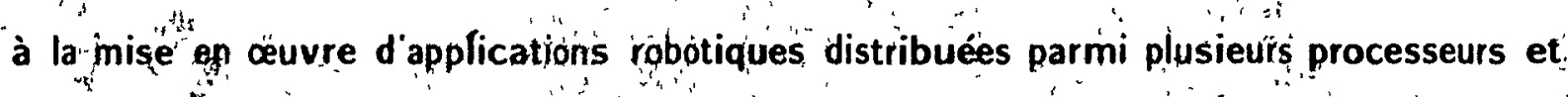
incluant plúsieurs robots et capteurs. L'ènvironnement offre deux miéthodés de communication qui diffèrent en complexité et en performance. mais qui présente une interface au réseau uniformé. La nóthade de communication pirimaire. «Session Layer $\gg$ (Couche de Session). est basée sur le «Open System Interconnect Basic Reference Módel»de l'Organisation Internationale de Nörmalization. Cette couche présente un format standärd pour intégrer les primitiveś de cơmmunication entre" tâchés d'un programmè d application complexe: ceci permet aussi ddasurei la compatibilité entre programimes écrits par differérents usagers pour qu'ils puisșent êtré intègrés dans une application de cellule de travail

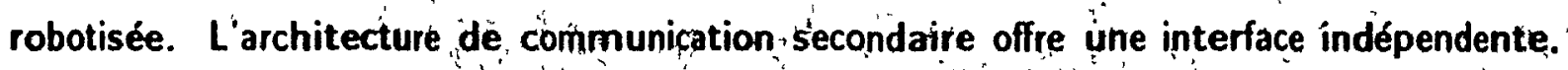
dun niveau inférieur mais trés efflicace pour la communication entre tâches. 


\section{Acknowledgements}

Financial assistance for this work was provided by the Natural Sciences and Engineering Research Council of Canada and the Conseil d Administration de la Fonds pour la Formátion de Chercheurs et l'Aide à la Recherche.

I would like to express my sincere thanks and appreciation to my supervisor. Dr. Alfred Malowany, who provided assistance. advice. and encouragement throughout the duration of my work: This work has benefited from the contributions of many people at our laboratory. In particular, I would like to tharnk my 'colleagues. Gregory Caryannis and Paul Freedman. who participated in the development of the Session Layer and who coauthored severat papers based on this research. 1 would also like to thank John Lloyd and Mike Parker for their comments in the Initial stages of this work' and Mike Parker for his programming assistance.

\section{(4) w5}

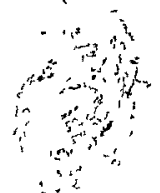




\section{Contents}

Abstract

Résumé

Acknowledgements

iv

Conitents

List, of Figures

Chapter 1 Introduction

1.1 Introduction and Motivation

1.2 Scope and Goals

1.3 Outline of the Presentation

1.4 . Research Goals of the Robotics Laboratory ..... $\ldots \ldots \ldots$

1.5 Implementation Environment $\ldots \ldots \ldots \ldots \ldots \ldots \ldots \ldots \ldots \ldots \ldots \ldots$

1.5.1 UNIX 4.2BSD Interprócess Communication Facilitiés $\ldots \ldots \ldots \ldots \ldots$

\section{Chapter 2 Interprocess Communication and Distributed.}

Computing

2.1 Introduction.

2.2 Distributed Systems

2.3 Synchrónous and Asýnchronous Communication

.2.4 Layeręd Communication Standards

2.4.1 The Öpen System Interconnect Basic Referencé Modèl

2.5 Summary

Chapter 3 Interprocess Communication and Distributed 
3.1 Introdúction

3.2 Programming Language Approaches to IPC in Robot Work Cells

3.3 Network Oriented Approaches to IDC in Robot Work-Cells

3.4 Backplane Bus Oriented Approaches to IPG in Robot Work-Cells

3.5 Summary

4.1 Introduction

4.2 Design Goals.

4.3 Design Constraints

4.4 The Session Layer Design

4:411 Qverview . .

4.4,2 Session Layer Architecture : $\ldots \ldots \ldots \ldots$

4.3 Session Layer Function Library .... . .

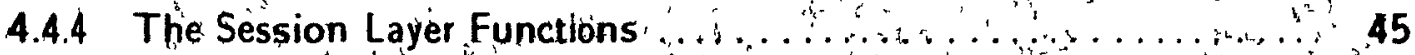

4.4.5 A Sample Séssion Layer Program . . . . . . . . . .

4.4.6. Additional Features of the ISO Session Layer Model ....... 55

4.5 Netwark Interface Library (NIL) Design .... 57

4.5 .1 Overview . . . . . . . 57

$4,5.2$ The Network Interface Library Functions . . .

4.5.3 A Network Sàmple Interface Library Program . . . . 65

4.6. Comparision of the Session Layer and NIL $\ldots . \ldots, \ldots \ldots \ldots \ldots \ldots \ldots$

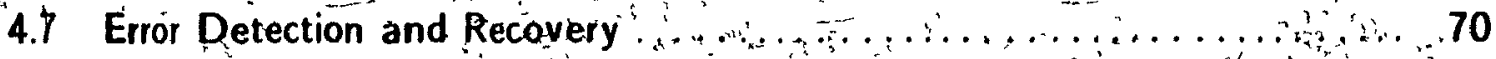

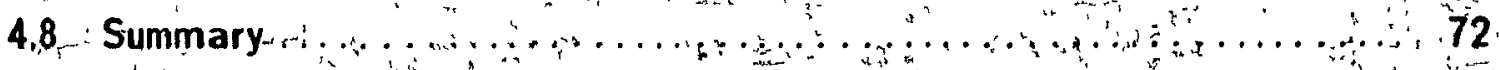


5,1 Introduction $\ldots \ldots \ldots \ldots \ldots \ldots \ldots \ldots \ldots \ldots \ldots \ldots \ldots \ldots \ldots, \ldots \ldots \ldots$

5.2 Performance in a Distributed System $\therefore \ldots \ldots \ldots \ldots \ldots \ldots \ldots$

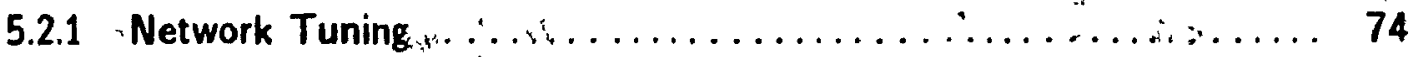

5.2.2 Network Contention........................... 75

5.2.3 Local versus Remote Communication $\therefore \ldots \ldots \ldots \ldots \ldots \ldots$

5.2 .4 Processor Load . . . . . . .

5.2.5. Message Length ............................ 77

5.2 .6 Program Overhead ................................ 77

5.3 ; Pérformance !mplementation ......................... 77

$5.4:$ Experimental Design..$\ldots \ldots \ldots \ldots \ldots \ldots \ldots \ldots \ldots \ldots \ldots \ldots$

5,5 Analysis and Results $\ldots \ldots \ldots \ldots \ldots \ldots \ldots \ldots \ldots \ldots \ldots$

5.5 .1 , Variation of System Load ........................ 81

5.5.2 Variation Between Host Computers ..................... 84

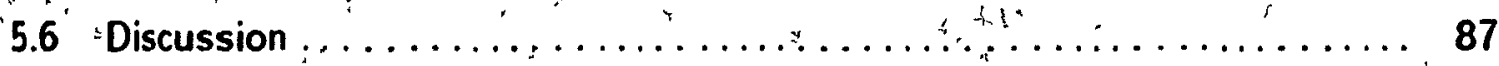

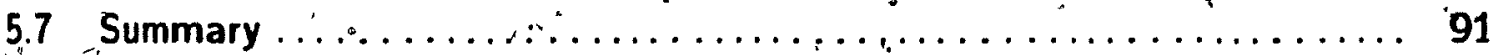

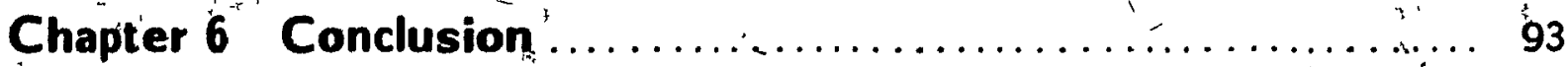

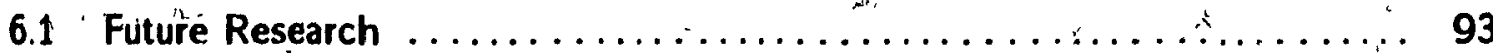

6.1.1. Operating System Resident Daemons .................. 94

6.1 .2 Reducing Overhead $x \ldots \ldots \ldots \ldots \ldots \ldots \ldots \ldots \ldots \ldots \ldots \ldots, \ldots$

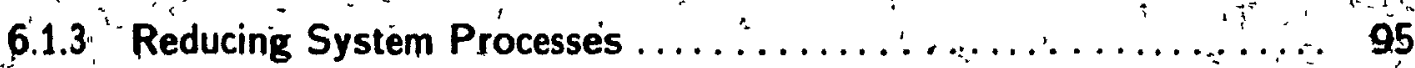

Rêterènices: 


\section{List of Figures}

1.1 Interprocess communication mediated by the Session Layer,$\ldots \ldots \ldots \ldots \ldots$

1.2 A typlcal CVaRL robó work-cell configuration $\ldots \ldots \ldots \ldots \ldots \ldots \ldots$

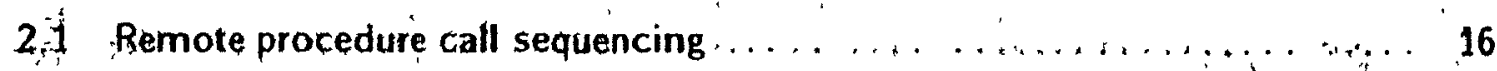

2,2 An example of a maitbdx architecture $\ldots \ldots \ldots \ldots \ldots \ldots$

2.3 The OSI Basic Reference Model for layered communication .......... 21

4.1 The three levels of the network interface $\ldots \ldots \ldots \ldots \ldots \ldots \ldots \ldots$

4.2 An example of an. Administrative Table $\ldots, \ldots, \ldots, \ldots, \ldots, \ldots, \quad 42$

$4.3 \times$ Session Layer architecture over a two host network $\ldots \ldots \ldots \ldots \ldots \ldots$

4.4 Arit example of a Dialogue Table .... $\ldots \ldots \ldots \ldots \ldots \ldots \ldots \ldots$

4.5 The Session Layer functions . . . . . . . . . . . . . . . .

4.6 Architecture of the example's communication structure $\ldots \ldots \ldots \ldots \ldots$

4.7 Session Layer Application Program 1 for Host A - Process A. . . . . . . . 52

4.8 Session Layer Application Program 2 for Host B - Processes B. C . . . . 54

4.9 Network Interfacé Library initialization functions $\ldots \ldots \ldots \ldots \ldots \ldots \ldots$

410 NIL commúnication architecture for create child .......

4.11 NIL communication àchitécture for creatè pqir. $\ldots \ldots \ldots \ldots \ldots \ldots \ldots$

4.12 NIL communication architecture for createchild remote $\ldots \ldots \ldots \ldots$

4.13 Network Interface Lobraty management functions

4.14 Network Interface Library termination function $\ldots \ldots \ldots \ldots \ldots \ldots \ldots \ldots$

4.15 Nit Applicatión Program 1 - Process A . . . .

4.16 NIL Apálication Program 2 - Processes $B$ and $C \ldots 67$ 
5.1. Configuration implementıng, both Session Layer and NtL links

5.2 Estimation of Session Layer and NIL message transmission times.

5.3 Parameter estimates of equation 5.2

5.4 Estimates of message transmıssion times.

5.5 Parămetề estimătes for transmission times.

96. Estımates of NIL messáge transmissıor times

5.7 Estımates, of Session Layer message transmission times

5 8. Raţio of Nilt to Sešsion Layer performances

5.9- Performance ratio for VAX/VAX host pair

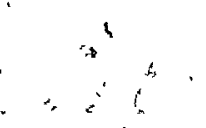




\section{Chàpter 1}

\section{Introduction}

\subsection{Introduction and Motivation}

\section{6}

Commercial robots were first introduced by Plannet Corporation in 1959. They were programmable devices: controlled by limit switches and cams. and capable of performing "simple : pick-and-place" and painting tasks. 'The development of róbotic units' employing servo systems for general path control was led by Unimation Incorporated during the 1960 ' $\mathrm{s}^{\circ}$ - Commercial robot controllers based on digital computers were realized in the early 1970's. with the introduction of T3 by Cincinnati-Milicron Corporation (Ayres and - Millè 83j:" This provided a convenient means of integrating external digital and analogue based equipment to the controller. allowing more complex tasks to be performed within the robot work-cell

During the $1980^{\circ}$ s. work-cells have evolved to include enormously varied and complex configurations. The single robot has been followed by'multiple robots. cooperat ing in three dimensional dynamic environments which allow them to accomplish complex and intricate taskśs. Intelligent computer basèd peripherals such as vision systems are be"ing employed for inspection of objects and robot supervision through motion coordination. Sophistiçated serising devices such as lasers and acoustic, ultrasonic. strain and pressure śensồs àre being incorpơrated for proximity determination. Controllers for lighting. motorized stages, and end effector tools are frequently components of state of the art work-cells 
[Goldwasser 84] Motion planning and adaptive control are essential to these complex configurations. custom expert systems have been employed to coordınate the various elements [Gonzalez and Safabakhsh 82]

Because of the complex nature of the tasks performed, centralized control is tho longer practical. The role of the robot controller as the nucleus of the work-cell has -been superseded. with control now distributed amongst many elements. The robot controller has become simply another peripheral device Work-cells have become centres of distributed computıng. networks of many devices This is exemplified by IBM's modular 7575 Man- '。 ufacturing System which is composed of arobot 'manipulator, a control computer. and a servo power module. The control computer can be linked through a network to any number of other host systems.

Inherent with the increased complexity of the robotic work-cell are the problems associated with any distributed computing system. The need for an easy to use interprocéss communication (IPC) erivironment which integrates individual elements both within and between work-cells is immediate. This structure must afford efficiency in its application while facilitating user acoess to any given element. In addition. device dependent features should ber made transparent to the user, encapsulated in such a way as to conform to a consistent interface.

In general, industry has been șlow in propọsing and implementing robotic workcell communication standards:- This might be attributed to the complex nature of the subject-and its wide range of applications. There are as many "standards" as there are robot manulàcturers. Communication remains largely vendor dependent: both hardware and software interfaces are frequently unique for each individual element. This uniqueness incurs high costs to the end user when interfacing elements. Incompatibility ánd installation protemsean sometimes be circumvented by purchasing all equipmènt from a single vendor. Frequently though. each system 'must reflect a particular set, of device requirements which are not met by a single manufacturer: In addition. companies tend to avoid single sourcing and purcháse, work-cell elements from: different vendors. Even if a manufacturer were to 
meet all the device requirements. thề products may not be the nosit cost effective

This situation is gradually changing with the adoption of the so called "open systems interconnect" philosophy put forth by the International Standards Organization, (ISO). An open system is one for which standards have been publishẹd. allowing system components from different vêndors to be integrated The iSO has propossed a heirarchical communications framework. called the Open System Interconnect Basic Rétetence Model (OSI). which partitions the tasks involved in communication over a computer network inito seven logical layers. Protocols describıng the function of each laygr have also been specified: The result of this development is a strong desire to standardize an industry that is rapidly" expanding. Standardization makes communicatıomore productıve by providıng a sţable hardware and software model:

One example of a robot communication stanidard based on the isO open communication reference model is the Manufacturing Automation Protocol (MAP) [MAP' 85 ]. proposed by General Motors. The MAP spefifications represent the first attempt at, a concerted effort on an international basis by major industries acting in conjunction-with professional societies to establish protocol profiles for automated manufacturing networks and to test and evaluate them in practical applications. The proposal appears to have

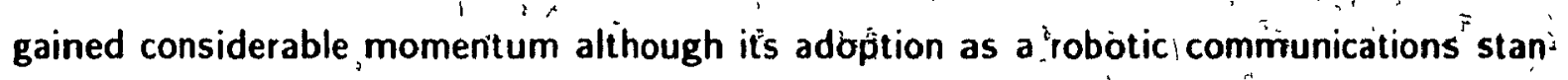
dard is not expected in the near future. Although recently available commencial systems supporting the MAP specifications are" well suited to large industrial applications such as if factory floor communications. they may not be attractive for full implementation in certain , research oriented environments. However, since the specifications are weill designed. it is advantageous to consider adopting any applicable features into a robot communications environment.

\subsection{Scope and Goals}

This thesis describes the development of a communication programming environment for the Computer Vision and Robotics Laboratory (CVaRL) of McGill Universitty. 
The CVaRL communications environment is a hierarchical structure which can be described with the OSI Reference Model. The OSI Reference Módel divides the tasks involved in the communication between two systems into a layered hierarchical structure composed of the Physical. Data Link. Network. Transport. Sessión. "Presentation. and Application Layers This model will be discussed in greater detail in Chaptè 2.

The environment, was expanded to inctüde two useful communication architéctures which vary in complexity and performance, and offer a consistent user interface to the network. The primary focus of this presentation to describe the design philosophy. architecture and implementation of a Session fayer which provides a standardized and straight forward means of effecting interprocess commùnication. The secondary communication architecture. which is provided by a software package, called the Network Interface Libràry. offers a flexible ànd very efficient but nònstandardiżed means of achieving ènd-toiend process communication.

The Sessiọn Laỹer pravides the user with a easy to use programming interface that facilitates access to the interprocess message pašsing setvices supported by the Transport Layer. The Session Layer appears to the user as a black box which links communicating processes and acts as antermediary in passing messages between them shown in Figure 1.1. Communication.services are presented to the user in terms of logical taps on the network called endpoints and logical channels between endpoints called. links. Network communication is a compiplex issue. and to achieve it in an efficient 'way often requires an in-depth knowledge, on the part of the user, of the low level aspects of network communication. The Session Layer alleviates this through a set of high lével communication primitives which allow the establishment. management. and termination of éridpoints" and links.

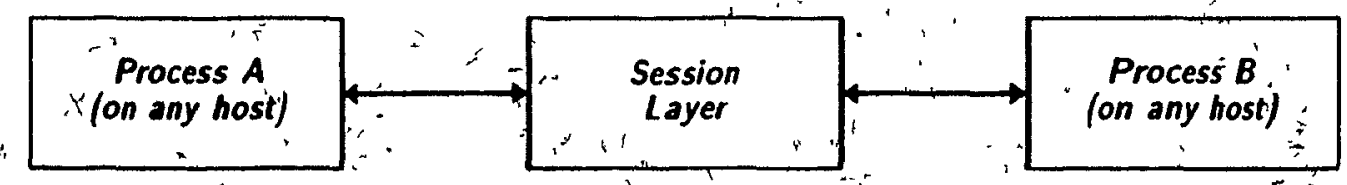

Figure 1.1 Interprocess communication mediated by the Session Layer" 
The Session Layer's standardizèd format aids in integrating interprocéss communication primitives into sophisticated application programs. It ensiłres compatibility so that programs that were developed independently can be stiared amiongst users and easily integrated into therr wark-cell appliçations: Ștandárdization is a most desiráble feáture to have in a research environment as it promotes productivity. Users do not have to mánatain their own set of communication routines and newiusers can benefit from an existing library of work-cell utilities.

Implementation of thè Session Layer required the development of set of network primitives to communicate with the transmission control protocol facilities in the Transport Layer. These primitives are available to the user in the form of an-interpocess communication library called the Network Interface Library. (NJî). NIL supports direct user. process to user-process communication: that is. interprocess communication that is not médiated by an intermediary such as the Session Layer.

Nill simplifies the implementation of 'jnterprocess communication applications by providing an interface to the system network which is easier to usè than that provided by the Transport Layer as well as by furnishing the building blocks necessary to implement some of the most commonly u'sed communication architectures. NIL provides lower level network interface than the Session Layer: it is not as powerful and is somewhat more difficult to use On the other hand the primary advantage of the library over the Session Ĺayer is iț increased speed. This package mây be used independeñt of." or in cơnjunction. with, the facilities provided by the Session Layer according to the user semands;

The goals of this presentation are tô survey methods of interprọess cọmmunication with an emphasis on those common in both génetal computing and distributed robotic applications: to discuss CVaRL's communication environment to describe the design añd implementation of a Session Layer and a Network Interfaçe Library suited to our envionment: to present an evaluation these of implementations: and to make suggestions for future research. 


\subsection{Outline of the Presentation}

1.3 Outline of the Presentation

The remainder of this chapter is devoted to discussion of the research goals of the robotics laboratory. description of the implementation environment, and justification for why it should be changed.

Chapter 2 discusșes of interprocéss communication in the context of distributed computing. Chapter 3 presents a survey of interprocess communication if the-context of distributed robotics ${ }^{\prime}$. Chapter 4 identifies thê interprocess communication services mostt Useful to our research community. and then describes' the design philosophies and architectures of our Sessipn Layer and Network Interface Library. In Chapter 5 we use qualitative and quantitative criteria to evaluate the communicationn services. Chapter $\mathbf{6}$ summarizes the presentation and makes stuggestions for future, research.

\subsection{Research Goals of the Robotics Laboratory}

At the present time, the principal focus of the ryobotio group at CVaRL is the inspection and repair of hybrid integrated circuits: The intent is to investigate the hardware ańd software requirements of a robotics work-cell whose purpose' is to visually inspect a target die and repair certain kinds of hybrid'circuit defects Related work is aimed at the inspection and repair of printed circuit boards. including the insertion, and removal of. components and the inspection of solder joints.

To this end, we are exploring certain aspects of distributed computing, especially hôw networking a set of work-cell elemients cạn influence réal-tintie perfórmance, and how to design algorithmis and data structures for a distributed environment.

\subsection{Implementation Environment}

In order to evaluate peyformance results and place them in their proper coritext. it is necessary to become acquainted with the implementation environment We can-char- 
acterize the computer network as mini and micro computer based, running a concurrent operating system with modest input and output requirements. The primary reasph for the distributed environment is to enhance performance through parallel processing. The labo": 'ratory "currently has twelve computers linked by a $10 \mathrm{MHz}$ "Ethernet local area network. The network configuration is composed of a VAX 11-780, three VAX 11-750's. two microvAX $\|{ }^{\prime}$ s. and six Sun System II's. All systems operate under the University of Berkeley at San Diego"s UNIX version 4.2 operating system (UNIX 4.2BSD). except for the VAX 780 which concurrently runs VMS 3.7 and EUNICE 3.1, a UNIX emulator.

A typical work-cell configuration of computer, robot, and vișion and othèr sensing systems is shown in Figure 1.2. One VAX 750 is dedicated to robot control applications while"the other systems are directed towards vision and the control of peripheral depvices.

Three robots are currently available in the laboratory: a Unimation PUMA 260. a Microbo ECUREUIL, and an IBM 7565 Manufacturing System: The:IBM system incórpo-, rates a gantry-type robot for light assembly and is programmed in its nátive language: $A M L$. The PUMA and ECUREUIL robots are both have six degrees of freèdom: the configuration of the PUMA is anthropomorphic whereas the ECUREUIL is cylindrical. Both the PUMA and the ECUREUIL share the same programming environment. RCCL. which replaces their respective native robot programming lànguages. VAL and IRL. $R C C L$ a librắry of rbbot control primitives written in C. was originally developed at Pứdue Univerisity [Hayward and Paul 83] and has since been enhanced and adapted for our research envitonment by" Cloyd [Lloyd 85].

\subsubsection{UNIX 4.2BSD Interpròcess Communication Facilities}

\subsubsection{1" Socket Communication}

The standard 'UNIX 4.2BSD distribution includes $a_{\text {. }}$ Trạnsport Layer protọcol called Transmission Cóntrol Protocol/Internet Protocol (TCP/IP) which providẹs a lów level network IPC interface [UCB 83]. [SRI 82]. [Tuthill 85]. All computers on the CVaRL 


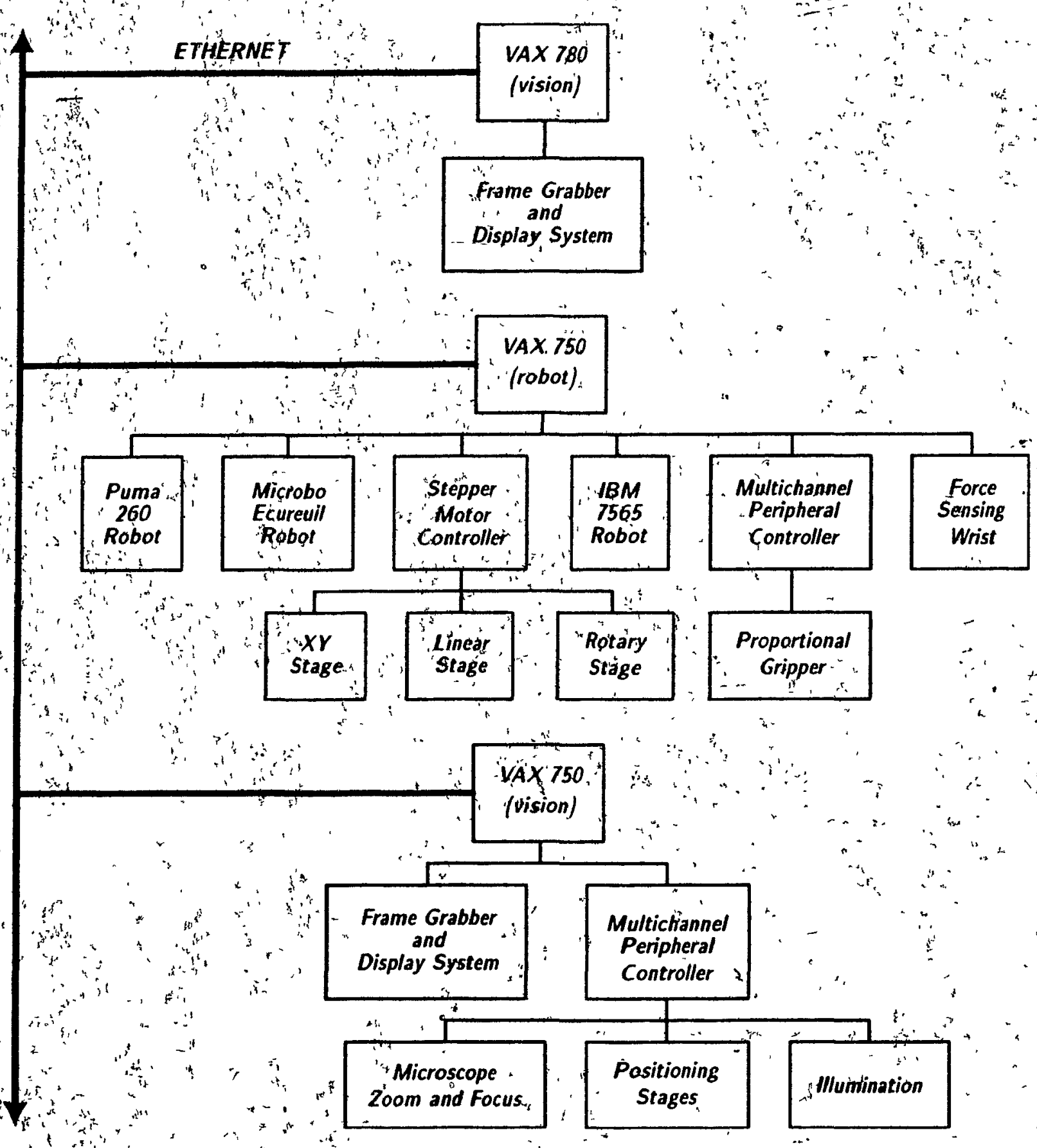

Figure 1.2 A typical CVaRL robot work-cell configuration

Ethernet support the TCP Transport Layer protocol: In this section. we will provide'a cursory review of some of the more important TCP facilities and demonstrate that although they are complete. they do not support interprocess communication at a sufficiently high usêr interface level. 
UNIX 4.2BSD šupports several communication domains which represent dif-

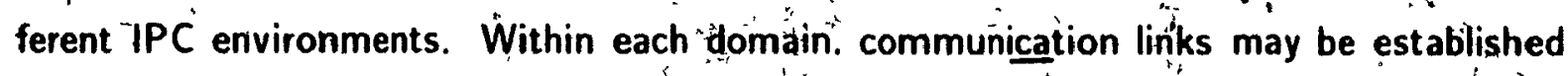
between endpoiñts known as sockets. Sockets are classified according to their abstráction type. which specifies the semantics of communication. In most applications there are two useful types of soeket services offered within the internet domain. datagram and stream... Datagram, services arre connectionless: the message delivery is not guaranteed to be sequenced in the correct order. reliable, or unduplicated. Stream sockets provide reliable. flow-controlled. full duplex data transmission over virtual circuits. A virtual circuit refers to

"' a communication link that may or may not be a physical channel between two processes.

- Distributed robotics demands reliable message transfer: so we will henceforth restrict our considerations to stream type sockets.

Sockets are cilassified as either active or passive. and may" bè either connected or disconnected. All sockets are created active and unconnected, by the UNIX function socket. A socket is made passive by binding it to a port identifier through the function bind. A pair of unconnected active and passive sockets may be cannected. establishing a reliable, flow-controlled. full duplex communication link referred to as virtual circuit. Connections are initiated from active to passive sockets by the function connect Passive sockets listen for and accept connection requests from active sockets. Data transmission ?between pairs of connected sockets is conducted by read, write.' recv and send.

A socket appears as a file to the operating system. When a socket is created. a socket descriptor, which is similat to a file descriptor, is returned. In establishing a connetction from an tactive to a passive socket, the active socket is referenced by this descriptor while the passive socket is referenced by its port identifier. This implies that the process requesting the connection must know the full port dentifier, that is. "the concatenation of the host identifier with the TCP port: which has been assigned to the passive socket. When a passive socket accepts a connection. it actually creates and accepts the connection atza duplicate socket: a descriptor of this duplicáte is returned. The original passive socket remains open and may listen for and accept additional conniection requests from other ac: 
tive endpoints. Thus, more than one apparent connection may be. estaplished to a socket. Data is transmitted or received àt a connected socket by referencing its sôckêt descriptor.

\section{5:1.2 Pseudo Terminal Communication}

In addition to the socket oriented approach to interprocess communication. UNIX 4.2BSD provides support for a device-pair termed a pseùdo terminal. A pseudo terminal is a master/slave pair of charactèr devices. The operating system views the slave device as it would any other terminal: all system software fờ controlling terminal device drivers can also be applied to the slave device. 'Data sent from a slave device servesias input for the master device and vice versa.

Pseudo terminals aŕe intended for conversationa/computing bètween programs that require terminals for standard input "and output. Prócesses communicate between pseudo terminals over the network. through söckets. Pseudo terminals are normailly used

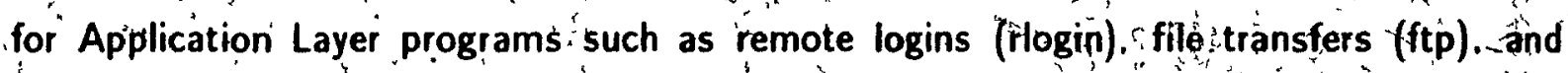
user-to-user phonéfacilities (talk).

\section{1:5.1.3 Justification for a Higher Lëvel Netwoonk Interface}

It is apparenit that the UNIX 4.2BSD IPC facilities provide an adequate means of achieving interprocess network communication. Howèver. sttaining: efficient network communication in a distributed processing environment is not a trivial task with these functions alone. Many programmers find the TCP IPC primitives difficult to understand and to work with. Programs using the TCP network primitives can sometimes be long it is not uncommon for a program which implements the communication architecture of a single work-cell application incorporating two robots, a vision system. ând positioning stages. to

"require over one thousand lines of program code. Furthermore some aṕplications demand a knowledge of system level programming to be efficiently implemented

These problems are compounded by the complex nature of the IPC communication structures that are often associated with sophisticated robot work-cells Couple $\therefore$. 



\section{Chapter 2 Interprocess. Communication and Distributed Computing}

\subsection{Introduction}

In this chapter: we review some of the approaches to interprocess communication from the general perspective of distributed computing enviranments. We describe the complex naturé of distributed systems and how communication is achieved. Two general categories of commúnication mechanisms, synchronous and asynchronoùs. are reviewed and examples of each mechanism are presented. Layeféd commúnication structures and the ISO's Open System Interconnect Basic Refence Model, an emerging standard for hierarchical architectures', are also discusšed.

\subsection{Distributed Systems}

A distributed computer system is a collection of processing elements which are phyśscally interconinected. controlled by system-wide resource managers, and capable of executing application processes in a coordinated manner. Coordination is accomplished. through communication and sychronization of the processes associated with each processing elementit. Factors which have influenced the development of distributed systems include performance. processing throughput. flexibility: reliability, resource sharing. decen-n tralization: and gröwth potential [Joseph 74]: 
22 Distributed Systems

Interprocess communication is a complex issue which is compounded by the fact that there is no unique approach to communication or synctironization. This problem is a result of the wide variety of hardware and software configurations which are possible.

The type of communication implemented is usually dependent on the amount of coupling present, that is. the degree to which the system 's processors share resources. The two extremes of processor coupling are tight and foose.

A tightly coupled distributed computer system is characterized by its, reliance on shared memory as an intermediary. with no facility for direct message transfer between processes, a single common operatıng system which coordinates and synchrọnizes interactions between processors: some degree of resource sharing: and processing power divided equally amongst the systems autonomous processors [Fathi et al. 83] Such systems have been built around concurrent programiming languages such as Concurrent Pascal |Brinch-Hansén: 75] and Modula [Wirth 77]. whictr asșurne that programs execute on a single processor and communicate through shared variables."

This is in contrast to loosely coupled systems whose processors communicăte without the benefit of shared memory. loosely cotupled systems are composed of autònomous computers which employ message based mechanišms. which are effected in accordance with network communication protocols and must the efore contend with such 'issuès as network access communication protocols and remote procedures. These systems are often controlled by distibuted operating environments which incorporate communication interfaces such as that provided by BSD 4.2 UNIX [Tuthili 85]. The early context of this, "work was data transfer over long-haul networks, it has since evolved towards distributed. systems and local area hetworks (LAN's). Both concurrent and distributed environments seek to fide communication details from the usert making remote operations resemble local ones.

The requirements of a robotic work-cell can be viewed from the perspectives of concurrent and distributed software as well as tightly and loosely coupled hardware. A 
23 . Syochronious and Asynçhronous Comminication

loosely coupled system offers greater flexibifity. However some elements in the work-cell must work together in a tightly coupled way. perhaps requiring specific kinds of interprocess communication in order to meet special constraints on speed and reliability it is desirable to presentr a simple and uniform programming interface which woufd hide the underlying aspects of communication. selecting the appropriate IPC approach is often difficult.

\subsection{Synchronous and Asynchronous Communication}

Interprocess communication can be classified as elther synchroñous or asyn-

chronous. A.synchronous protocol is onje whose çommunication primatives employ blocking techniquès to regulate the flow of traffic. A primative is said to be blocking if once a message has been transmitted, the source process is suspended until an acknowledgement has been received from the destination. This is analogous to the function call implemented in most programming languages. Blocking techniques are most often used for commiand/response or query/status signalling ['Silverman 84 ].

Blocking protocols have their advantages as well as their disadvantages: Since only one message can be in transit at a given time in a synchronous system. extensive message queuing at both the source or dêstination is not necess ary. affording simplicity in its. implementation. However blocking protocols can be inefficient in that they restrict parallè processing. When a process sends a message, it must wait until the entire message has been transmitted and the remote prócess has acknowledged its receipt. Since distributed components are nót always in close proximity the time to transfer the message could be significant, Timeout features must be included the sert and receive primitives to pievent, indefinite blocking should the destination program be unable to respond programming

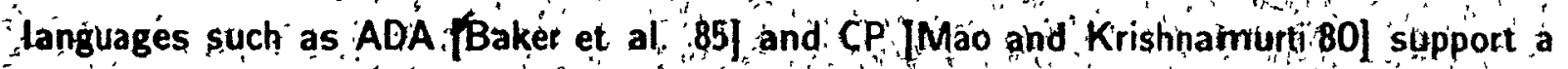
disconnect mechanism that allows the programmer to control the acknowledgement delay.

Another model of synchrónous méssage passing is the Remotre Procedure Call (RPC) [Birrell and Nelsón 83]. [Holmgren 85]. The RPC model allows user programs to 
23 Synchronous and Asynchrohous Communication

invoke remote procedures in a manner which is similar to that for local procedure call models. When invoking the tocal procedure call. the caller places arguments in a procedure call buffer contiol is passed to the procedure, and then eventually returned to the calling procicdure. At that point. return parameters are extracted from the procedure call buffer With the remote model, RPG services manage message passing to and from user processes by incorporating dedicated 'slave' servers that perform specialized IPC services for 'master' user programs:- The user therefore avoids direct use of the low level system. primitives for communication. An RPC is like most system function calls. invocation blocks the guser program until the procedure returns. as shown in Figure 2.1. This decreastes the power of the computing environment somewhat by removing the possibility of concurrent sender/receiver actuvities. Despite this shortcoming. RPC allows the user to approach distributed programming in a very. sımple way.

As discussed in [Andrews and Schneider 83], one can also provide a commúnications facility by embedding special IP, $C_{3}$ primitives iñ a programming language. A number of attempts have been magde to to design general purpose distributed programming languages with IPC primitives that use the Remote Procedure Call model. Three examples. ADA, PLITS and CSP are compared in [Shatz 84].

Synchronous protocols are suitable for tightly-coupled distributed programming environments, but are often excessively restrictive in loosely-coupled concurrent environments, Additionầ flexibility car bé provided by asynchronous or message pašsing protocols. [Stankovic 82] Under asynchronous protocols. the sending process' does not have to w for the receiving process to acknowledge the message, suspension of programs pending. acknowledgement of transmił̧ted messages is therefore not réquirèd. However. this might nécessitate the queuing. of message transmissions. The extra complexity can be buried deep within the operating system or hidden from the user through the use of dedicated servers, Nonetheless, asynchronous IPC tends to demand a higher level of programming sophistication from the user than synchronous IPC. For example, the' user must anticipate the possible blocking of transmissions at the source or destination. 
MOST A

Local Process Local Daemon

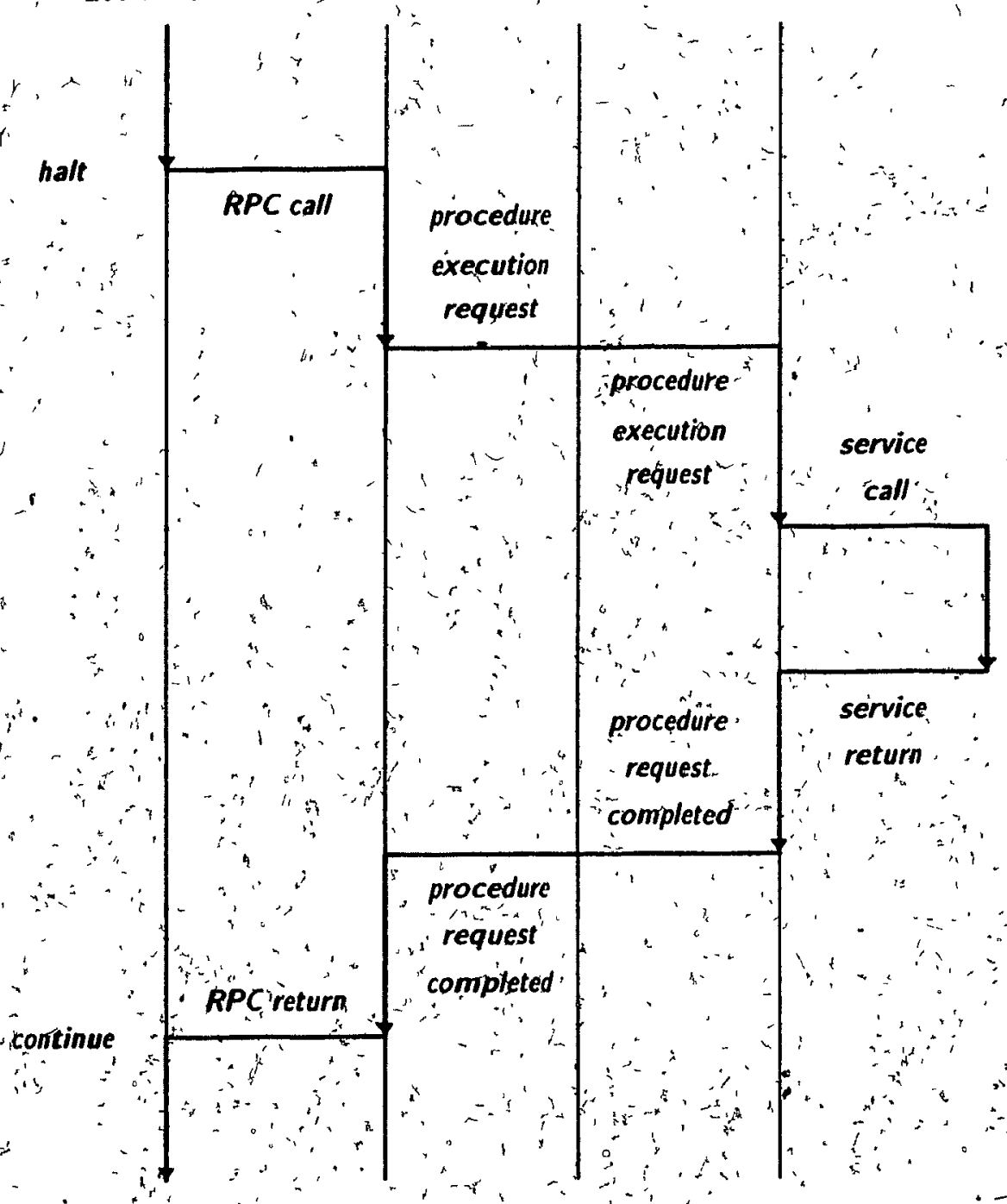

HOSTB

Remote Daemon

- Remote

Procedure

Figure 2.1 Rémote procedurêt call sequencing

The simplest model of asynchronous user/server interaction is dialogue, whereby the user 'requests' services which are 'granted' by the server. This model differs from the Remote Procedure Callin-that a master/slave relationship is not established and thus. the server may not always be available. Of course, the diąlogue model presupposes a facility for reliably sending and receiving messages. In general. we find two kinds of message passing services: the datagram, and the virtual circuit: Datagram services are connectionless; 
23 Synchronous and Asynchrö̌nous Communicatior

connection is established temporarily, only for the duration of the message transmission Following transmission. the communication link is broken Datagram message delivery is not guaranteed to be sequenced in, the coirect order, reliable. or unduplicated. This is sometimes called 'transaction-oriented' coommunication. becausé each datagram represents' a complete and independent transaction

In contrast are virtual circunt services, which maintain the commication link until the user dectides to terminate it. Streàm sockets provide reliable. flow-controlled."full duplex data transmission over virtual circuits. Eacti transmission is acknowledged by its recipient: In the event that-an acknowledgement is not returned to the source, within a fixed timeout period. the transmission is automatically repeated: thus. message delivery is guaranteed. This requires that transmissions must be buffered at the source and that com. ṕlicated error detection and recóvery protocols mușit be, in place. The associated overhead. results in a slower service than that provided by datagrams. Virtual circuit communication" "services are sometimes referred to as 'end-to-end" or 'connection-oriented".

Another user/server 'model of asynchronous communication is the mail service. "This service represents a general class of IPC models that are frequently encountered in tightly coupled systems which have multiple processors utilizing shared memory IFaro et al. 85]. Programs exchange infọmation in the fơrm of mail messages which are sent to and "retrieved from 'assignèd mailboxes, in common memory illustrat'ed in Figure 2.2. Associăfed with each processor is a send and recerve mailbo which resides in a bank of common memory that is memory which shajed by afl processors on the system. $A$ process sends a messäge to a local server which packetizes the data and transfers it to the appropriate receive mailbóx. The destination processor is signalled when the message has been deposited. It respond's by issuing a receive command to a local server which then fetches the message.

Mạiling cañ appear to be vẻry responsive but it actually involves significant operating system overheàd. Incoming and outgoing mail queues must be managed: reading a message involves reviewing the headers of numerous irrelevarit messages until the required 
Processor I

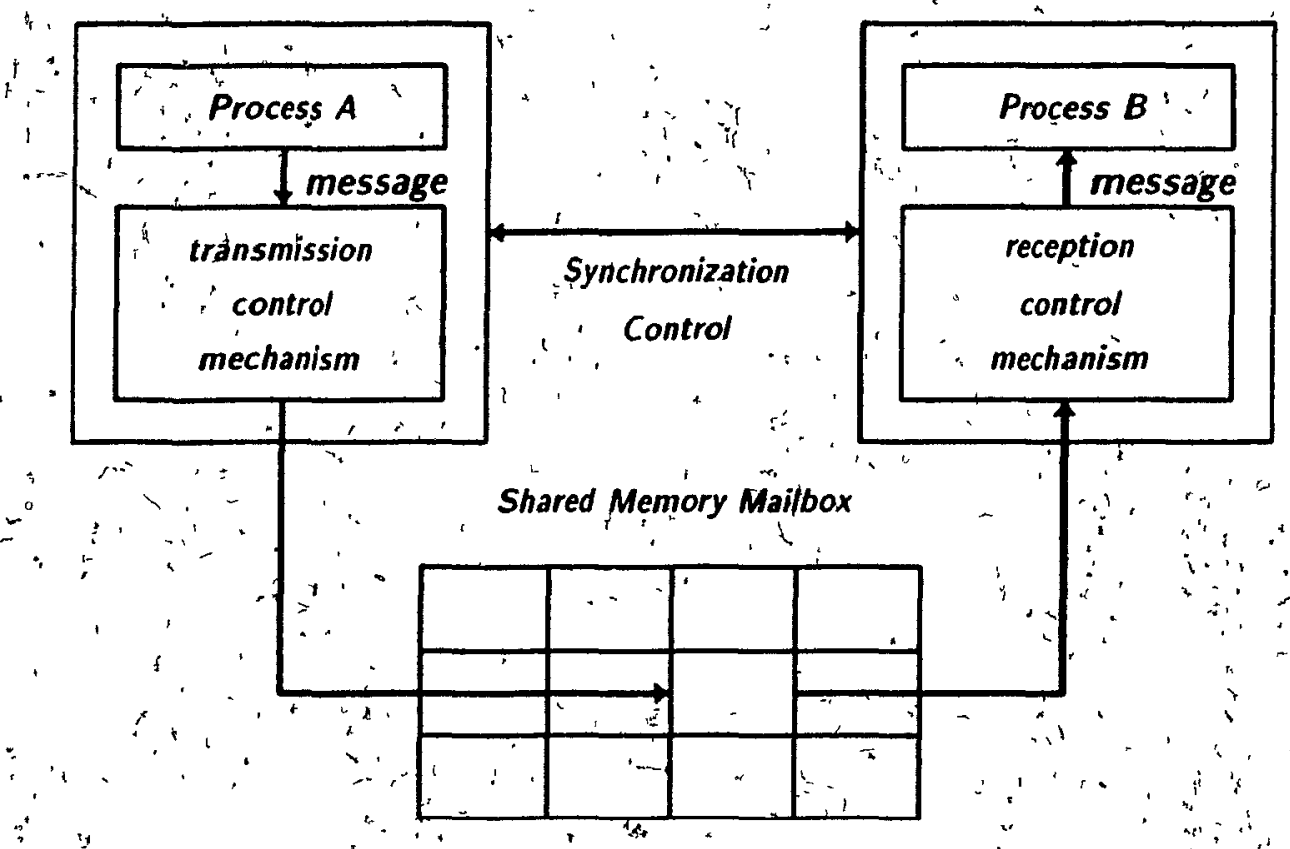

Figure 2.2. An example of a mailbox architecturè

, One can be located: some systems packetize the data: and the problem of common memory contention muśt be addressed. Typicạlly, only one piocessor, can ácćess the common memory at any one,time. creating a bottleneck. Even when multiport memory is used. cóntention is still a serious' problem. This problem is further aggravated by' the 'fàct that when transferring data between processors, the common memory must be accessed both when depositing and retrieving the message. This tends to restrict the number of processors which can be effectively supported by a single operating system.

Shared memory systems that are implemented in loosely coupled networksystephs mpope a significant strain on the network as well as higher operating system and computing overhead than that of bus oriented systems. An example of a shared memory network system basied on XENIX is discussed by Devarakonda |Devarakonda et al, 85].: The implementation mechanism parallels that of the bus systems but there is the addi- 
24 Layered Communication Standards

tional burden of message packetization. error detection. and correction that is inherent to the network transport protocols

\section{2:4 Layered Communication Standards}

Layering serves to decompose the mechanism of communication into smaller sub-tasks. Each layerprovides services to the layer above and below it, in a way which is independent of how the services are performed. This permits the independent implementation of each layer. thus making it possible to integrate products from different vendors. By clạssifying communication services intố standardized layers: modifiçătions can also be made to one layer. for example. to improve efficiéncy or speed, without affecting the operation of other layets.'

However, not everyone agrees with the layered approach. - Detractors warn of excessive communication overhead, and point to functions: such as flow. control and error détection. which are duplicated in each layer [Clark 82]. High level protocols must some; times undo the error recovery, of, lower levels, as for example when, a message' must be completely rétranśmitued despite retransmission of message fragments.

Opponents to layering believe thăt better performancé can bę achieved by building IPC into a distributed operating system which is supported by special software resident on each host' the software is 'sométimes bundled as separate ssérvers', or simply incor. porrated into the kernel intself. "The actual exchange of messages, 'usually of fixed length. is carried out using a simplédata link layer or network datägram protocol. Others have added more sophisitication to this basic service by providing explicit management of virtuat circuits. User processes obtain IPC services by sending system request's to the kernel over a link which was dedicated to that process when it was first created. The underlying network service guarantees delivery of all messáges.

As a rule. all the designs described thus far ignore the aetual contents of messagès. However. there are some designs which provide different types of communication 
services for different kinds of messages. For example. a distributed system om offer datagrams. - virtual circuits. and a special 'expedited' service for high priớrity mèss łyes. In additión. the message tỳpe could dictate whether synchronous or asynchronous pretótồ aje employed. Some designs automatically provide differént IPC services on the basis of message type. Andrews 82]. Rather, than leaving the selection of the IPC manism to. the user. a set of message types is provided añd each one is passed in a different way.

\subsubsection{The Open System Interconnect Basic Reférence Model}

The Open Systems Interconnection (OSI) Basic Reference Model of the Internationatkstandards. Organization is a strong conntender to become the standard hierarchical communication framework [Zimmermann 80]: [ISO 7498]. The QSI model originated in Eutope wtrere it quickly gained widespread acceptance. In "North America however. IBM's System Network Architecture [Hoberecht 80] is the accepted standard because it provided a means for integrating IBM's mainframe computer products.

The OSI model breaks the major functions involved in communicating between two systems into an orderly sequence of seven layers [Tanenbaum 81]. Standards specify the services and protocols of each layer. The Bàsic Reference Model. defined by ISO 7498 and adopted in 1984. is shown in Figure 2.3.

The Physical Layer [ISO 8802,4] is the-lowest level of the Reference Model.. It I specifies the electrical and mechanical aspects of the communication hardware as well'as the functional control of the data circuits. This layer activates,-maintains, and deactivates the physical connection.

The Datą Link Layer [ISO 8802.2] establishes." maintaiñs, and 'releases data links: It also performs point-to-point error and flow control of frames.

The Network Layer [!ऽC 8473] is responsible for network routing, switching. segmenting. blocking. error recovery, and flow control of packets. It is in this layer that 
Layer

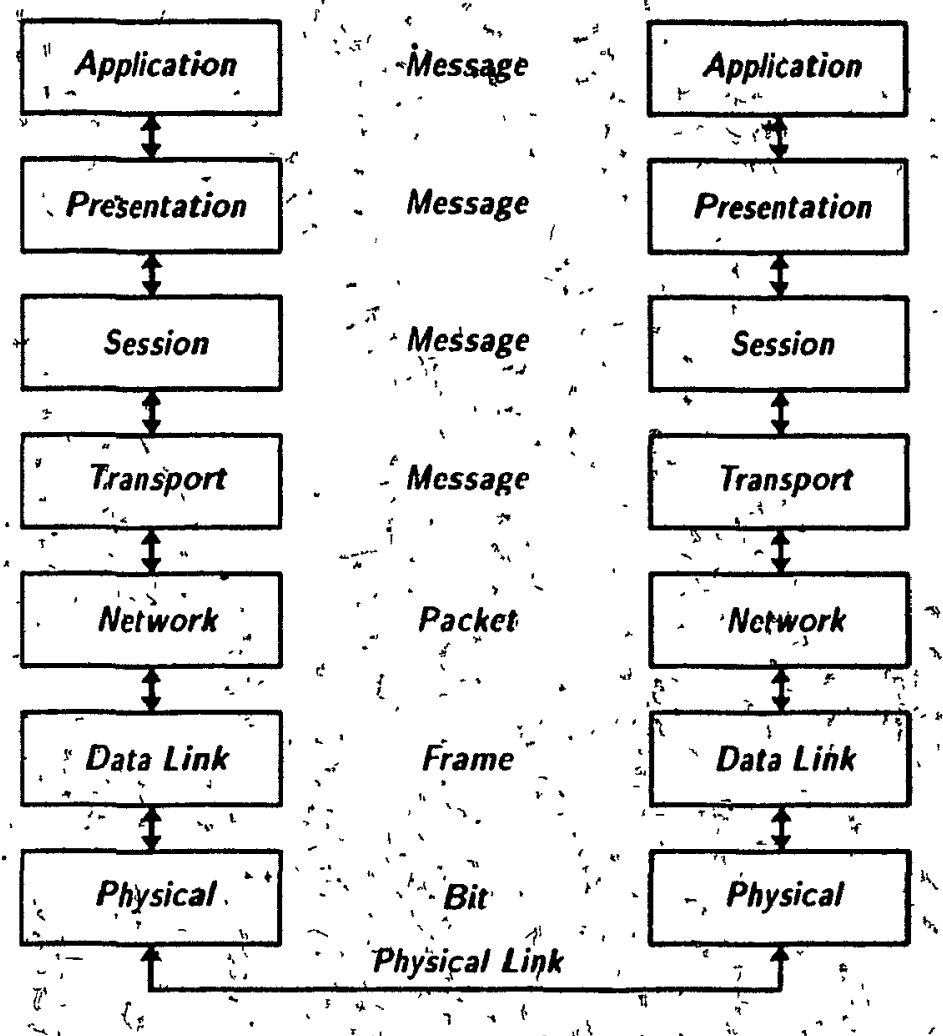

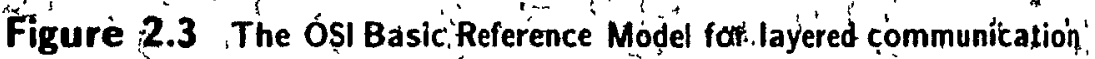

the network traffic is managed by either relaying dăta up to an application process ô down along a physical network patr.

The Transport Layer [1SO 8072], [ISO 8073 ] furnishes the base for high-level protocols by effecting data trạnsfer and end to-end reliability control. "This layer provides varioüs communication services to the Session Layer. multiplexes messages over logical connections, and segments data fọr the Network Layer.

The Sesśion Layer [ISO 8326]. [ISO 8327] establishes and terminates logical links bétween processes and manages the dialogue over those links An important function of this layer is to synchronize data between the application processes.

The Presentation Layer [1SO 8649 ] is respónsible for the interpretation and 



\section{Chapter 3 interprocess Communication and Distributed Robotics}

\subsection{Introduction:}

- A manufacturing work-cell should ideally consist of one or finore robots. programmable workpiece positioning devices, sensory elements süch as vision and haptic systems, and a programming environment which includes facilities fór intèrprocéss communication both within ardọ between work-cells.

In this chapter. we discuss three basic approaches to interprocess.communication in a distributed robotics environment. We bègin with a discussion of programming language approaches to robotic IPC, followed by system developments aimed network systems, and end with a review of backplane bus systems.

\section{2, Programming Language Approaches to IPC in Robót Work-Cells} Most programming fanguagés' developed for industrial mạnufactưring applications fail to address the twin issuess of communication between work-cells and communication within work-cells [Gruver ęt al. 83], JBonner and Shin 82]. This' makes interfacis $\mathrm{g}$ the necessary intelligentit sensol and actuator systems of the work-cells very dificult. Until recently. most commercial robot controllers provided only very primitive communication facilities such as paralle! and serial ports which served as interfaces for binary input/output. 
3.2 Programming Langúage Approaches to IPC in Robrot Work-Cells

a display terminal. and perhaps a teach pendant: and no host controller interface |Jarvis 84]. [Nagel 83].

"This situation has bécome inadequate for two maiñ reasons. As programming tasks become more difficult. there is a need for a richer programming environment thán that which is provided by the native robot controller. Secondly. the need for more flexible or intelligent behavior has meant the incorporation of the robot itself into a larger framework under the control of other hosts. These systems are sometimes referred to as flexible manufacturing systems.

The simplest solution to the problem has been the development of special software to link an external host cómputer to the robot confroller through its terminal port: This enables robót software to be developed on an external host and then downloaded to the robot's controller via an RS-232 or remote control link. Examples of wireless links based on infrared frequency shift key signalling have been preșented by: Dwivedi and Pearson [Divivedi 83]. [Pearson and Green 84].

Alternatively, more complex designs with varying degrẹs of supervisory ability have beén implemented. based on dedicated host controller links. For example terminal emulation programs which send commands to the robot controller in the robot s native language are described by Carayannis and Michaud [Ċarayannis 82] [Michaud 85]. These programs serve to make the robot an integrat part of the distributed environment. and also "maké it pośsible to program the robot, using cross development facilities, in languages tother than the native one of the controller. An advantage of working on an external host system is the availability of powerful debugging and simulation facilities.

Some designs have sought to bypass the native programming environment en tirely by directly çontrolling the joint servos. Weiwill now review some of these programming language éfforts.

A set of process level communicạtiôn and synchronization primitives for an 11. integrafed multi-robipt system is described in [Shin and Epstein 85]: Five categories of

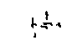


3.2 Programming Language Approaches to IPC in Robot Work-Cells

industrial prócesses are identified: independent: loosely coupled. tightly coupled. serialized motion and work coupled: specific communication strategies are developed for each. Each process manages a set of tasks, a task being the smallest element of control activity. Inde"pendent processes can simply "señd state update messages to tasks they have in common. With loosely coupled processes. the actions of one depends upon the other. Thus, they must quèry each other. or a common task. for state information. Tightly coupled processes wse a master/slave control approach: the slave always acts in accordance to the master. and returns a status message after each directive. in serialized motion processes. one or more processes must sometimes be performed before another can -begin. Communiçation is used for synchronization and event signalling. Finally, with work, coupled proce:ses. each one must maint zin a state description of all thè others: once a piocess completes.a task. it must broadcast the state change and then wait until all scate descriptions hâve beí. updated before re iuming execution

A modula: command languag: íor industrial robots. called $L M A C$. provides message "passing facilities for communication and synchronization of processes in a multi-robot industrial syștem [Bonin et al. 83]. To próxide the communication between processors. "a' 'LAN is structured' as a four-layer, hierarchy: nètwork. transport: session. and application. LMAC has been implemented on a set of LSI/11's with the prográmming language PASCAL.

-A dialect of PASCAL with new IPC primitives for robot programming is described in [Baird et al. 84]. The" target work-cell consists of a single PDP/11 control computer linked to two robots and two-visiơn systems via RS-232 șerial lines. Communication between the controller and a device is modelléd as an asynchronous series of commands and replies. There are also language primitives to allocate and deallocate the devices. The actual IPC within the controller is based on the message passing primitives provided by the RSX óperating system.

Programming of robot baśed manufacturing cells using ADA is discussed in [Volz ét al. 84]. They identify issues concerning the software aspects of robôt cell control 
33 Network Oriented Approaches to IPC in Robot Work-Cells

and how ADA addres'ses these issues. Included in their 'discussion is ADA's ability to manage large complex softwáre systems. the efficiency of code pròduced for real time applications. multitasking. interprocess communication and task synchrónization. portability. and program debugging in a real time environment. A distributed run time ADA package called DARP. which extends communication and task synchronization across machine boundaries. is curfently" under development [Fisher and Weatherly 86].

Instead of extending a báse language , one can also construct a procedưre library to perform specialized services. One example of this is $\mathrm{RCCL}$. a library of $\mathrm{C}$ functions for robot programming [Hayward and Paul 83]. [Lloyd 85] A servo program resident in the : kernel of the supervisory computer delivers setpoints to the individual joint controllers at prescribed intervals. Motion requests from the user program are translated into joint motions and then queued for transmission over a buffered high speed parallel link.

$\because \quad$ Finally, we review two second generation robot programming languages which have recontly been developed. These designs attempt to present a truly integrated 'system level design. AML [Țaylor et al. 82] is.IBM's answer to intelligent robot programming: all señsing and control is managed by a Šeries/1 computer. Unimation's VAL II. JShimano et a), 84] provides extended facilities for network communication and senisor interfacing. The robot controller has a port dedicated to supervisory control over a LAN. There is an additional high speed serial port for online modifications to the planned path of the robot from an external computer. When the external alter mode is active. the system sends messages to the external computer once every control cycle. to ènquire if changes should be made to the planned path. VAL III is expected to be released in late 1986. It is reportedly compatible with such networks as MAP. SECS III, and Intel's Bit Bus ànd has facilities tó. integrate a high speed vision system [UNIMATION 86].

\subsection{Network Oriented Approaches to IPC in Robot Work-Cells}

A Session Làyer for a LAN intended for robotic manufacturing cells has been described by [Bruno et at. 84]. MODIAC consists of clusters of 28001 microprocessors 
33 . Network Oriented Approaçhes to IPE in Robpt Work.Cells:

linked by a high speed serial bus. A distributed operating system called MODUSK l. Garetti. et al. 82] manages ạctivities within each cluster through shared memory. A Virtual Network Operating System is planned for inter-cluster process coordination.

the concept of an stivity, controller fờr a multiple robat work-cell is developed in: [Maimon and Nof 83]. The activity controller serves to sequence as well as synchronize the actions of multiple robots sharing tasks and auxiliary devices. such as feederș. In an assembly work-cell. One application is presented in [Maimon 85]. "wherein two robót's and. auxiliary devices execute asynchronous concurrent tasks: both robots are controlled from a VAX 11/780 host computer running UNIX. and are programmed In $C$.

A rietwork for dıstributed robotics. based on three host computer systems liaked by an Ethernet [Metealfe and Boggs 76] LAN is described in [Goldwasser 84] and [Goldwasšer and Bajcsy 83] An expert system reșides on êch host and is responsible fờ visíón. control. or haptic functions. The haptic expert provides an interface to a robot equipped with a sophisticated articulated hand. The hand consists of three fingers, each with two joints and three tactile, pads. For each firiger. separate processors are used to dive the joints and process tactile information. "Communication among the experts hierarchical. Each level can issue orders to experts on the lével below and pass responses back to the one immediately above. There is also a facility for high priority message passing.

In the context of LAN oriented developments. a network intefface designed for supervisory control associated with Unimation VAL II robot controller has been described by' Shimano [Shimano et al. "84j." The interface has three layers: the "bottom", which performs physical input/output, the "middle", which serves to multiplex multiple messages: for transmission over single link and the top which associates logical addresses with input/output devices. These layers do not directly map onto the OSI reference model for example, the "bottom layer is a combination of Physical and Data Link layers.

A layered approach to networking within a robstics environment based on the ÒsI model has been described by Faro and Messina [Faro and Mesșina 83]. IFaro, and 
$33^{\prime}$ Network Orientéd Approaches to IPC in Robot Work Cefls

Messina 84]. Within a work-cell they suggest á simplified meśsage passing facility based on shared memory A transport layer is maintained for associating devices with addresses. and a session layes is proposed to provide high level communication services sueh as opening "and closing of channèls, and managing data transfer ovèr the channels. A single host within each work-cell is designated master and serves as a gateway to the supernet which is based or redundant busses. Overall. three kinds of módules are jdentified to support robot caoperation within and between work-çells. one, for messagépassing one for remote jọb execution. and one for file management" of the three. "only the message passing module would be requited everiywhere.

The concept of networking work-cells within a factory and the Factory' Area . Network (FÁN) are explored in [Holland 83]. The author identifies a hierarchy of commúnication needs which parallel the hierarchy of devices within the factory. At level-1. the device level, messages are exchanged over dedicated point-to-point links for binary sensing and servo control. At level 2. messages are used for synchronization and data exchange between device controllers. Ethernet LANs are employed at the physical level to effect message transfer. At level 3, the work-cell level, message passing is in response to operator commands' such as production monitoring and data logging. Finally. at level 4 ;. we are concerned with factory wide data processing.

One example of a FAN is Allen-Bradley"s Distributed Network Architecture, A single data highway or LAN links distributed devices on the shop floor such as computerized númerical control (CNC) and robot stations, and gateways are used to tie networks of 'supervisory subsystems to the data.highway. A flexible manufacturing work-cell based on Allen-Bradley equipment is described in [Hanlon and Westori 82]. Two programmablè controllers are used to manage a robot arm and a CNC machine, and they are linked by a data highway. But the data highway is used for more than communication within the workcell: a remote file server, and a network supervisory station, are also connected:" The LAN is a high speed baseband serial bus, withia çontention based protocol. When a station on the net desires to transmit. it must wait until the current nẹtwork master has transmitted 
all of its messages. All active stations are then polled: mastership of the net is granted to the one with the highest priority which has something to transmit. - In fact. the polling is done twice:-first, all high priority stations are polled. and then stations with 'normal' proority.

Most manufacturèrs of fexible aútomation still provide próprietary solutions 'tó the conimumications problem, but this situation is changing. One recent development is MAP. a set of manufacturing automation protocols promoted by General Motors |Leopold 84]. [MAP 85]. [Kaminski 86]! MAP is a layered communication standard for linking distributed elements within a factory. The MAP specification is based on the communication. structure defined. by the Open Systems Interconnect (OSI) layered architecture |Zimmerman 80]. The OSI Reference Model was developed by the International Standards Organization (ISO) to provide a framéwork for connecting open systems (an open system is one for which standards are published), allowing system components from different manufacturer's to be inter-connected.

MAP is rapidly becoming a de facto standard in factory automation, "and has already gained widespread endorsement. MAP's physical transport system utilizes the IEEE's token-passing-bus network standard. although the Honeywell Plant Management System uses an Ethernet [Iversen 85]. Eventually. MAP will incorporate all seven layers of the OSI model. A pilot implementation which links 200 data acquisition, units monitoring 10,000 setsors at the General Motors car assembly plant in Oshawa. Ontario is described in [Storoshchuk and Szabados 83].

The token-passing-bus media access controlis also used by IBM's SNA ständaŕd and is favoured ovep Ethernet in a pure industrial application äs it is better suited to real time applications. The token-passing-bus protocol controls when an element can hạe acciss: to the network. This is based on a predefined order which is established to correspond to real time events in the manufacturing process. This is in contrast to Ethernet, which relies on collision detection with randomized backoff, that is, all parties wait a random, amount of time after a colfision. to resolve network contention. Ethernet is nat capable of message 
3.4 Backplane Bus Oriented Approaches to IPC in Robot Work-Cells

prioritizing and is generally better suited tơ applications where real time performance is not required!

Despite MAP. Ethernet is still a very popular LAN technology. For example. many manufacturers of Automatic Test Equipment (ATE) such as Fairchild and Zehntel havé develaped Ethernet; compatible networks. to link programming, testing. and repair statións [Milne 83]. In a distributed robot system for assémbly described by Barthes and Zavidovique. a vision system, a robot arm. and a planning modulé commúnicate via message passing over a custom Ethernet LAN [Barthes and Zavidovique 81].

A. distributed approach to complex robot systems based on mesša passing is described in [Harmon et al. 84]: the target problem domains are an experimental auttonomous vehicle and an automated welding work-cell. The-authors define three classes of subsystems: sensors.. which translate raw data into symbolic information. controllers. which translate symbolic plans into commands. and knowlèdge bases. which 'reason about symbolic information añd prepare plans. These subsystems are connected by a LAN in a bus topology, and interact by passing messages with specific formats. Each message has a source, a priority. and a body. The body can be of type plan. that is, a command or of type report. which is a response. A more elaborate message formatting scheme is described in [Harmon 83] and [Harmon ànd Gage 80]. in connection with a layered robot to robot communication. A message content layer formats information to be communicated using three fields. The first js called the address. which contains the identifiers of the source entity and one or more destinations. The second field is called the content, which contains the message itself and a context for the message. The third field, the message state, contains message parameters indicating message'type, priority. and the 'state' of the source entity.'.

\subsection{Backplane Bus Oriented Approaches to IPC in Robot Work-Cells}

In contrast to loosely coujpled networks are tightly coupled multiprocessor common bus systems. Severat examples of such systems are now described. 
34 Backplane Bus Oriented Approaches to IPC in Rớbot Work-Cells

A log loading robot crane is describèd in |Kärkkainen and'Mainninen 83]. A set of three 68000 microcomputer modules are configured as a sensor processing unit. a coordination unit. and a servo processing unit. They are linked by a VME bus and lcommunicate via shared memofy. The coordination unit serves to reconcile the sensory information with the desired goal state The servo unit is the heart of the system, and it superyises the robọt crane. a sensory subsystem, and the man/machine interface. The sensory subsystem is a daisy chain of sensors. each having a single chip microcomputer interface [Kärkkäinen 83]. The link to the servo unit is a high speed_RS-232 serial line.

The-design of another multi-microcomputer network for generalized robotics control. consisting of four 68000 microprocessors. is described in [Plessmañin] 83]. Data exchange between nodes takes place at the Data Link leveliover a custom paraltel, bus. Synchronization is performed via an interrupt schème.

Another example is the CHORUS system, developed at the National Rèsearch Council of Canada [Green 83]. which is based on the MULTIBUS. Communication is via message passing primitives suppliéd by the HARMONY opperating system [Gentleman 83]. A sample application is described in [Elgazzar et al. 84] to locate. recognize. and then insert regularly shaped blocks into matching holes. The robot application task on host ănd the vision application task on añother host communicate for mutual synchronization and exchange of data.

A multi-robot real time environment with intelligent sensors requirès an efficient communications mechanism which promotes parallelism. partitioning of tasks, and recon-.. figüürability. Two parallel bus designs which meet these requirements are RAPIÖbus [Wînilis and Sanderson 84] and REPLICA [Ma and Krishinamurti 84].

In [MacWilliams et al. "84]. a high speed seriąl bus from Intel called Bitbus is described. The authors suggest how a Bitbus could be used to link related physical devices such as "multiple joint servos, and then a Multibus, to connect distinct but cooperating subsystems within à work-cell composed of robot and vision systems. Finally. they propose 
34 Backplane Bus Oriented Approaches to IPC in Robot Work-Cells

separate work-cells be intérconnected via a factóry wide Ethernet:

In this context of tightly coupled systems. we also mention a project underway at Carnegîe Mellon University to dẹvelop an autonomous laṇid vehicle [Elfes and Talạkdar̂́. 83]. A set of slave processoirs dedicated to drive train control and sensing systems for sonar. cameras. and proximity devices are linked to expert modules fesident in a central processoror. The experts share information via shared memony but communicalon between a slave and its master is via message passing. This is managed by a small real time kernel resident in the each prociessor.

Ar industrial workicell for intelligent assêmbly is described in [Stauffer 85]. One robot acts as a materlal feeder for two others which weld pads on ignition modules. A vision system is used to accurately locate the pads for welding. The material handling robot acts

- as the master: it communicatès with the wètding robots via eight bit parallel ports. and with the vision system via a seríal port.

A simple robotics testber for small scale laboratory experiments is described in [Wainwright and Moss 85]. The direction artd speed of each robot joint servo is controlled by pulse width modulation. using astom robot controller." Communication between the controller and the supervisory host is bver an 8 bit parallelled bus.

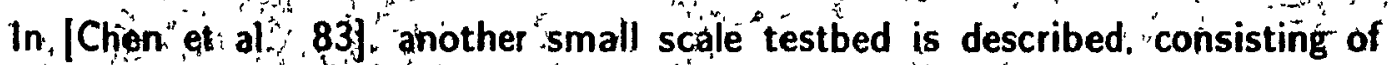
two robot arms with separate controllets and a master controller with a voice recognition front end. The master passés messages to the two arms via dedicated serial lines to their controllers.

An experimental testbed for coordinated robot. and vision work, which is described in [Makhling 82]. is based on a Westinghouse vision system and an Olivetti robot with two arms. Communication between the two LS! /11 based systems is via high speed DMA message transfers. Extensions to the Olivetti roboti programming language SIGLA to support vision reląted corinmânds is âlso described. 
A testbed at the Jet Propulsion Laboratory for generalized bilateral coñtrol of two robot arms is described in [Bejczy and Lee 84]. Each robot is controlled by à set of three National Semiconductor microcomputers which communicate with èach other over a shatred parallel bus.

A tightly' coupled three level hierarchical network of sixteen 8086 based micro:computers is desctibed in [Alford and Belyeu 84] for the coordinated control of two PUMA "arms.' The multi-arm coordination computer transmits new position commands to each robot via high speed block transfers to intermediaries called piédiction computers. The prediction computers use a simple handshaking protocol to issue new setpoints to the robots" joint controllęrs.

\subsection{Summary}

Although the need to standardize communication mechanisms is apparent. re-:action from the robotics industry has been slow and limited. A survey of relevant work suggests that few attempts,attempts have been made: the survey presented was more a review of research activities than industrial efforts. Each of the approaches presiented satisfies a particular aspect of interprocess communication in a distributed robotics environment. Depénding on the system requirements, one has the choicé of a programming language versus an operating system approach complimented with either a loosely coupled network system or a tightly coupled multiprocessor system. The literature indieates that the most favoured häechanism of interprocess communication is message passing entrenched in a hierarical framework the reason for this is, probably because of the general purpose hardware and software configurations found in many laboratories. Opur work is based on this approach and in the following chapter we describe an easy to use means of achieving efficient IPCin this type of environment.
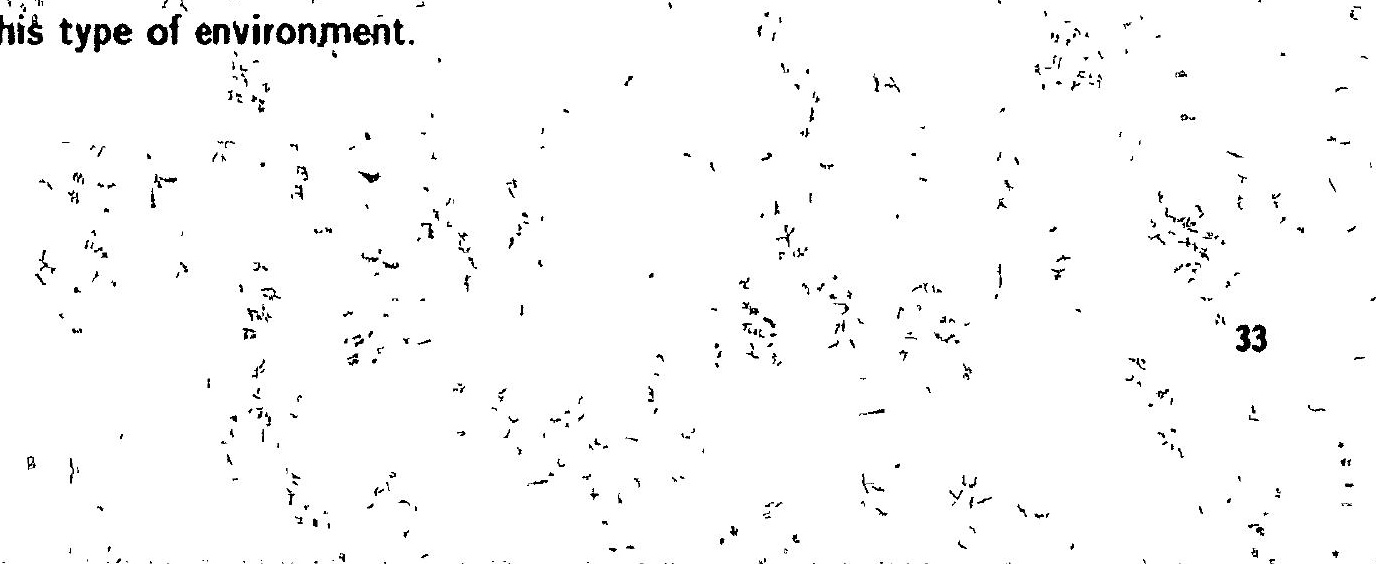


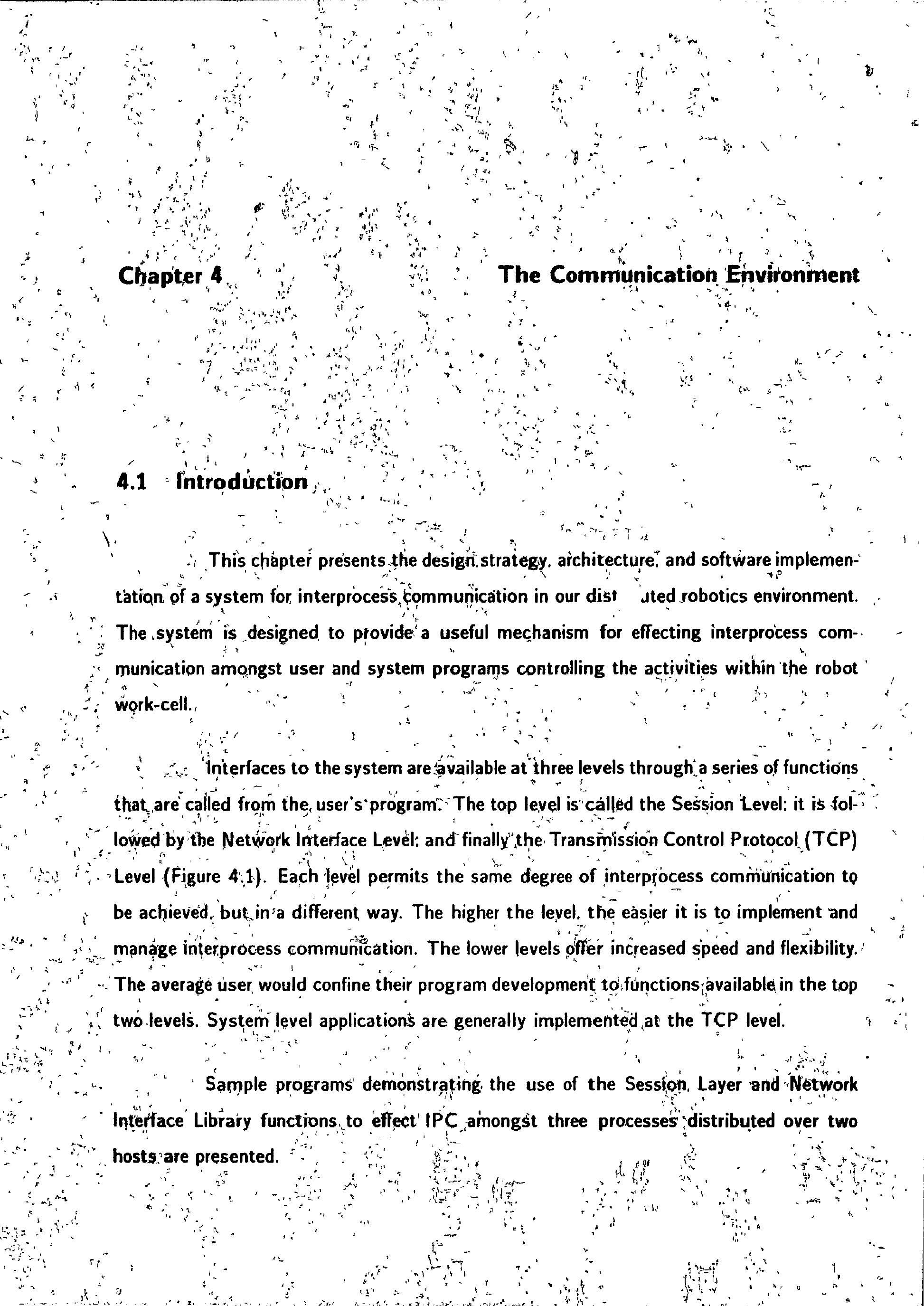




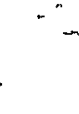

(1)

i.

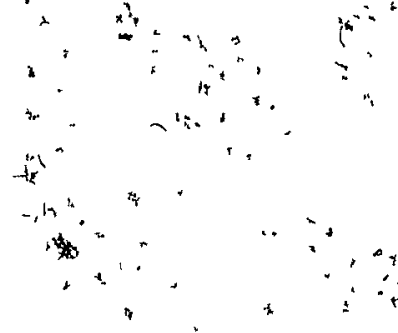

Level

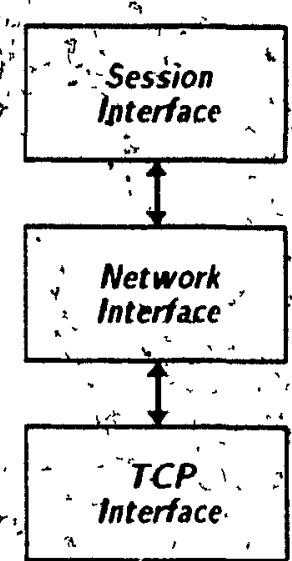

Figure 4.1 The three levels of the network interface

\subsection{Design Goals}

The communication environment has the following design goals:

Flexibility: Since a research environment is dynamic. ifis important that new equipment can be integrated easily and in a consistent way.

- Modulárity: Robotic work-cells are composed of a number of distinct elements, each "of which is responsible for its own subset of tasks required for the work-cell's oper-r ation? The model of communication used should complement the modular nature of the work-cell.

- Ease of use: The communication interface should be simplistic, consisting of a small , number of easy to use functions that are independent of which work-cell elements are communicating. "The user should be shielded from all aspects of the low level IPC mechahisinto

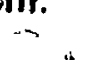

$=0$ 


\subsection{Design Constraints}

The following design constraints emerged from an analysis our our robotics en-

ip vironment

Interprocess communication will Be achieved at the level of the operating sys têm rather thañ through a prógramming langùage. Allthough. a lạnguage approach eases implementation of communication primitives and generally more transportable than an operating system. we consider it unwise and impractical to restrict all programming to be carried out with a single language in order tochieve IPC. BSD 4.2 UNIX, which includes TCP is well suited to this application as it supports the necessary low level IPC primitives to build a high lèvel comṃunications enviroñment.

The physical link between distributed devices will be accomplished using a inetwork approach rather than a common. bus, "A $10 \mathrm{MHz}$ Ethernet will be used to link the work-cell elements. Although the backplane bus offers increased speed through shared memory and signalling. it represents a less flexible design; The backplane limits the number of connectable elements, they are restricted to a cammon focation, and there arề always problems with system integration. As VLSI pushes down the cost of équipment, it is bêt. coming more practical to distribute intelligence within the work-cell and tevices together with'a network.

like most of the network based distributed applications surveyed. we favor the user/server padradigm. In this schéme, each host incorporates a communication server that provides the user with set of communication primitives which are independent of the programming language being used. 'We favar asynchronous message passing over more restrictive alternatives, such as the synchronous remote procedure call or mailbox type systems. Utility processes controlling the work-cell will reside on different host computer systems. IPC will be used to emphasize co-ordination of sèts òf parallel tasks, rather than simple data exchange. Users will logically structure their jobs: the vision programis will execute on one host. robot programs on other hosts, and so on. With this distributed 
43 Design Constraints

3

intelligence: orè would expect. for the most part. that meșìges transferred bẹwèen elements will be short and infrequent. keeping the amount of network traffic between entities lowi - Thus, the high bandwidth" provided by a backplane bus system is not nëcessary.

$\because$ The communication framework will be based on the ISO's Open System In.

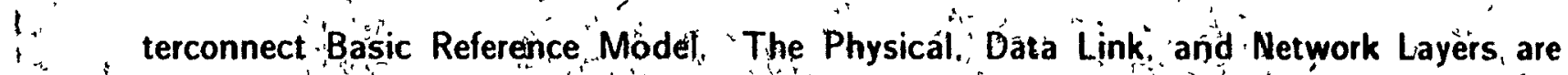
$\therefore$ "implemented by the Ethernet: the Transport Layer will be provided by TCP: a Session Layer will be implemented and the Presentạtión and Application Layers are inherent to BSD 4.2 UNIX.

The networks iser interface will consist of a partial implementation of the Session Layer Model. We anticipate that by poviding only the most useful features of the OSI model we will achieve both simplicity through a high level interface. and more efficient IPC. By adopting OSF principles. the design can take advantage of the applicable specifications and protocol algarithms.

In implementing the Session Layer. the integrity of UNIX will be preserved:

?. "the implementation will not involve any modifications to the operating system kernel. We recognize that this may have negative effect on the communication performance charactefistics but expect that this will be more than offset by a less complicated design and a shorter implementation time. In addition activity within the work-cell is presently bound by the speediof the robots and positioning devicés, as well as by vision processing. In our applications, the rate of IPC is at least an order of magnitude faster and is therefore not of prime concern: However, we would like to minimize the overhead associated with the Sêssión Layer.

Working with UNIX means that the $C$ programming language is the logical choice for the Session Layer implementation 'We shall also follow UNIX conventions whéte. possible, as for example with error handling, to maintain á consistent system interface to the user., The Session Layer will be compatible with programming language such as Foprtian. Pascal. LISP or assembly, whose object modules can be linked with C. 

- providing a métens of process synchronization

supporting error detection and recovery

The Session Layer hides the low level aspects of communication from the usen. appearing as a black box which links processes and acts as an intermediary in exchanging messagès. Our Session Layer interface consists of coimmunication endpoints and links between the endpoints. An endpoint is a Session Layer logical tap an the nefwork which is unquely associated with aser assigned name: The user references endpoints by specify. ing only their logical names links are referenced by pairs of names. identifying source and destination endpoin̂ts The Session Layer translates the logical names, into physical network addresses, which are specially ordered concatenations of host addresses and network port identifiers.". "These addresses are expressed in a stàndàndized form of external data rèpresentation that is necessary for different languages, operating systems. and "machine architectures to be able to communicate It is the responsibility of the Session Layer and not the user to identify on which host an endpoint resides: the user only has to identify the endpoint's logical riame. An important consequence of log içal addressing this is that machine indeperidence of Session Layer processes is supported

Beçausê the Session Layer manages dialogue over the links, it can be uséd to synchronize entities residing in the Presentation and Application layers as well as in user programs. This is necessa in coordinating tasks within the work icell.

A necessàry functiơn of the Session Làyer is error détection and recovery: both, inter and intra host error conditions must be considered. Fori, example. the Session Layer must respond to the loss of a host on the network so that links to thät host may be carefurly terminated while continuing to manage communjeation among the remaining hosts.

\section{A.2 Session Layer Architecture}

The Sassion Layer structure on each tiogst is hieraretically organized into two 2 等, 
tiers: the top level is occupied by an Admınistrative Server and the lower level by Dialogue Server. The Session Layer is complemented by a set of Session Layer primitives which are linked to the user's application program. These functions bridge the servers and user processes. providing the user with a means of creating and deleting endpoints. establishing and terminảting communication links between endpọints. and transmitting and receiving messages over those links. The Administrative Server is responsible for managing endpoints while the Dialogue Servers link the endpoints and coordinate dialogue over the links.

The Administrative and Diałogue Servers as well as the Session Layer primitives. are discussed below. Additional technical details" can be found in [Gauthier et al. 85].

\subsubsection{Session Layer Administrative Server}

The Session Layer Administrative Server is responsible for managing èndpoint information. This includes maintaining recôrds of current endpoints and sharing this information with servers on other hosts. In addition. the Administrative Server keeps the Session Layer viable through inter-host error detection and recovery procedures.

The Administrative Server maintains a table. which is used to map user speçified IGgical names to port identifiers of passive sockets:- This table makes the port identifiers of passive sockets available to servers across the network. allowing the user ta endpoints by their names only. The table is updated through the user program by either appending descriptions of newly created endpoints or deleting those that are no longer needed. This includes removing entries created by user processes that have subsequently" terminated. These table entries. which we call 'abandoned', result from the user programi aborting unexpectedly or terminating without first having removed endpoint *descriptions from the table. Upon termination of a process, any associated TCP ports and open sockets are freed by the operating system: the corresponding table entries must be removed by the Administrative Server.

Separate Administrative Servers reside on each network host. Through interserver communication. the tables are maintained to be identical, each containingentries 
for endpoints across the network. Multiple servers offer speed and iedundancy' which hefps guard the Session Layer against collapse in the event that a host becomes lost to the network. that " if it crashes or its network interface becomes disabled, The servers also. exchange Session Layer status information and therefore play an important rọle in error rècovery" procedures:"

Eight data fields are required to completely describe an entry in the Session Layer Administrative Table (Figure 4.2). The server uses the first three fields of the table, the logical name assigned to the endpoint by the user process. the TCP port number assigned by the operating system, and the name of the host on which the endpoint resides. to map the name of an endpoint onto its socket address. The socket address is the concatenation of the TCP port number with the host's Iriternet address, which is derived through a system call by the server based on the host's name

The fourth field of the table, the user identification. specifies who ovins the endpoint: The motivation for this will be described later.

In maintaining the:Administratıve Table, the server is esponsible tor ensuring that 'abandoned', endpoint entries are deleted, This requires the inclusion of the next 'two table fields: the identification number of the process that created the endpoint, and the endpoint type The endpoint type may be either reserved. which identifies a permanent endpoint. or user. which identifies a endpoint whose entry should be removed from the table when its user process terminates. Reserved endpoints are intended to be associated with procésses controlling work-cell utility modulés. For example, assume that a system utility process exists that controls an XY stage and it's endpoint s logical name is stage 1. A user would simply establish a connection from one of their processes to stage 1 andd control the stage by sending and receiving messages to and from it.

The remaining two fields are useaful only to the Administrative Server that was involved in the creation of the endpoint. These fields specify the process identification. number of the Dialoguge Server. a child process of the Administrative Server which is ded- 
44 the Session Layier Design

icated to the endpoint, and the socket descriptori of the link between the Administrative and Dialogue Servers.

\begin{tabular}{|c|c|c|c|c|c|c|c|}
\hline $\begin{array}{c}\text { Endpoint } \\
\text { : Name }\end{array}$ & $\begin{array}{l}\text { TCP.Port } \\
\text { Number }\end{array}$ & $\begin{array}{l}\text { Host } \\
\text { Name }\end{array}$ & $\begin{array}{l}\text { User } \\
\vdots \text { idd }\end{array}$ & $\begin{array}{c}\text { Process } \\
\text { ild } \\
\end{array}$ & $\begin{array}{l}\text { Endpoint } \\
\therefore \text { Type } \\
\end{array}$ & $\begin{array}{l}\text { Dialogue } \\
\text { Socket }\end{array}$ & $\begin{array}{c}\text { Dialogue } \\
\because 14\end{array}$ \\
\hline$\therefore$ robpt. & 1029 & curly & $\because 0$ & 2343 & reserved & $\because 5$ & 2344 \\
\hline vision & 1031 & Jàry & 120 & 2345 & $\therefore$ user & .6 & 2346 \\
\hline inspect & 1023 & larry & 120 & $\therefore 231$ & user & 7 & 2021 \\
\hline st.sge.1 & 1027 & curly & 120 & $\therefore 345$ & reserved & $64^{\prime}$ & \\
\hline
\end{tabular}

Figure 4:2 An example of an Administrative Table

The Administrative Servers safeguard against the duplication of names the Administrative Tables. Priof to updating its table the server must verify that the requested name is not alreadg entered in the table. To eliminate the possibility of name duplication resulting from concurrent actions of Administrative Servers on distinct hosts. the servers adhere to a synchronous inter-server table updáte protocol. Only one server may initiate changes to the table at any time. A server requires permission from the other servers before proceeding with the addition of rames to the table and must notify the other seryers of the changes in order that their tables may be updated.

\subsubsection{Session Lạyer Dialogué Server}

The Dialogue Servèr is responsible for managing communication links. whicti includês establishing and terminating connections between endpoints, and copordinating

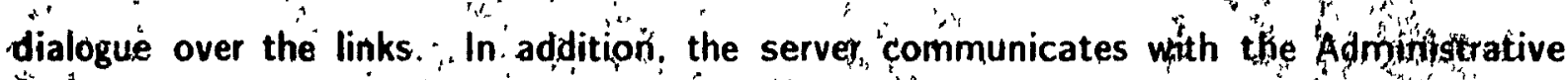
Server and other Dialogue Servers to exchange both Séssion Layer messages as as státús information.

A user program which consists of multiple processes majy have several Dialogute Servers associated with it. Each process involved in $I P_{C}$ has a single endpoint, with an associated dedicated Dialogue Server. These Diajogive Servers act as intermediaries in
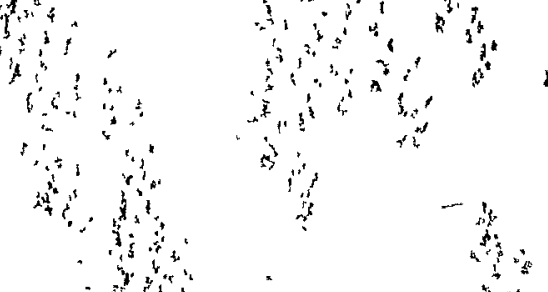
coordinating dialogue between endpoints. An example of the Session Layer architecture depicting an established session between a user prócess and a system utility pracés's over a two host network is shown in Figure 4.3. The exchange of messages is mediated by the two Dialogue Servers.

Host a

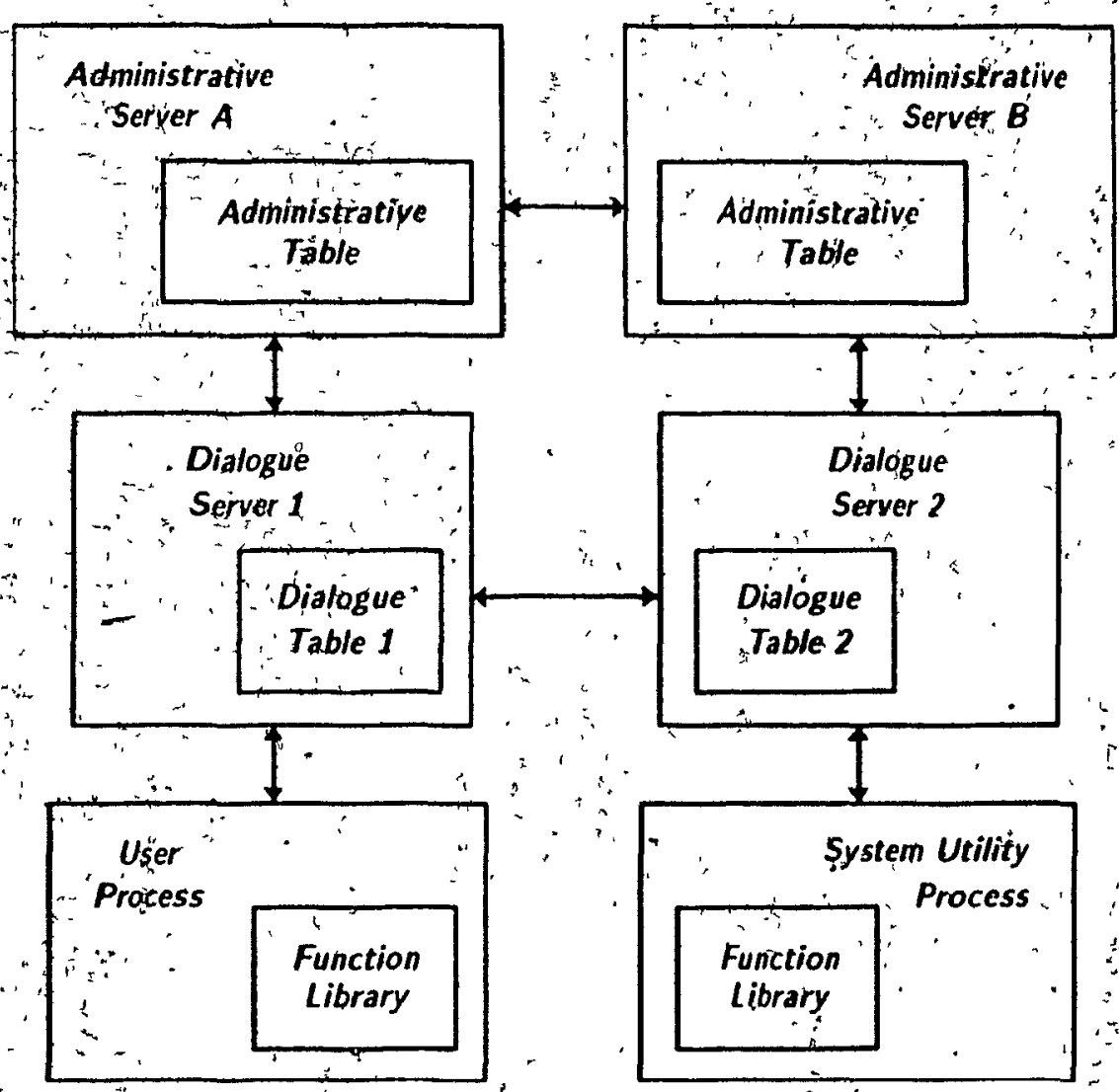

Eigure 4.3 Session Layer aŕrchitecture over a two host network

Each server maintains a Dialogue Table. shown in Figure 4.4, which describes the links established to thè Dialogue Servers of gther endpoints. Five data fièlds are required to describe a table entry. The first two fields. destination logical name and socket descriptor. mape the name identifying the destination endpoint of a link onto its socket descriptor at the source endpoint. This information is used by the server to identify the link over which a message is to be sent or received. The last three fields. messagé pending. signal enabled. fand signal number are used by the Dialogue Server to manage theireception of messages. 
Message pending tells the server that has read and interpreted the message at that endpoint to be data which will eventually be passed on to the user process! The signal enabled and signal number fields tell the server if the user proces should be interrupted upon the receipt of a messagè ánd if so. which interrupt signal should be used.

\begin{tabular}{|c|c|c|c|c|}
\hline $\begin{array}{l}\text { Destination } \\
\therefore \text { : Aame }\end{array}$ & $\begin{array}{l}\text { Socket } \\
\text { Descriptor }\end{array}$ & $\begin{array}{l}\text { Message } \\
\text { Pending }\end{array}$ & $\begin{array}{r}\text { Signal } \\
\text { Enabled }\end{array}$ & $\begin{array}{l}\text { Signal } \\
\text { Number }\end{array}$ \\
\hline vision' & 7 & 0 & 1 & $128^{-1}$ \\
\hline lighting & 6 & 0 & 0 & 29 \\
\hline $\bar{X} \bar{Y}$ stage & $8:$ & 1 & 1 & 30 \\
\hline
\end{tabular}

Figuré 4.4, An example of a Dialogue Table

The 'Diblogue Server communicatês with three different entities. the Administrative Server, the associated user process, and one or more other Dialogure Servers. Messages received by either Dialogue or Administratives Servers are always in the format of a command followed by any relevant data. Received messages must be read and parsed to determine the appropriate action and handling of any data that follows. For example. a message received by the Dialogue Server could contain status information from to tocal Administrative Server indicating that one of the network hosts will be going off line. The Dialogue Server would then inform the user process of the pending termination and perform an orderly shutdown of any affected communication links.

\subsubsection{Session Layer Function Library}

The Session Layer Function Library provides the user with an interface to the Session Layer Servers. The library is linked to the user program and executes in the user's process space, . Functions are provided to create and delete communication endpoints, establish and terminate communication links between endpoints. and exchange messages over the links. ", The functions provide a useful high lével network interface by building upon the IPC isystem calls provided by UNIX'4.2BSD. The user is not precluded from 
corcumventing the Session Layer and mixing lower level IPC function calls with Session Layer primitives. This might be necessary in instances where the speed of the Session Layer service is insufficient.

Some of the Session Layer functions must adhere to protocols to ensure that the various servers' tables' residing on different hosts are properly updated and deadlock situations do not occur. These protocols are a consequence of the general nature of the Session Layer and are part of the penality that must be paid when such a general purpose architecture is adopted. In applications such as this. master/slave paradigms are often employed. "Depending on the situation. these protoçols may be symmetric or asymmetric. In a symmetric protocol. either side may be the maśter while in an asymmetriç protocol, one side is always the master. Irrespective of the symmetry of the master/slave model. there. is always an asymmetry in establishing the initial connéction between the two processes. Under UNIX 4:2BSD. TELLNET, is an example of a "symmetric protocol which is used for remote terminal emulation. FTP is án example of an asymmetric protocol that is used for file transfers [Sun 85].

\subsubsection{The Session Layer Functions}

The Session Layer functions can be dividếd into three. classes: initialization routines, mahagement routines, and termination routines. The Session Layer functions" and their associated parameters ate summarized in Figure 4.5. A sample application of the Session Layer in which three processes commuriticate over two host computers is presented in Section 4.4.5.

\subsubsection{Initialization Routines}

- Init_session
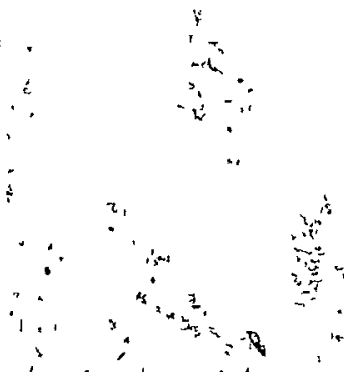

Init_session initializes a set of logical names in the Administrative Server Table. The Administrative Server is then responsible for ensuring that the logical names. entered
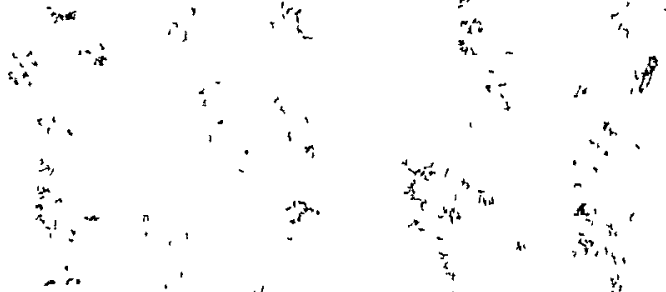


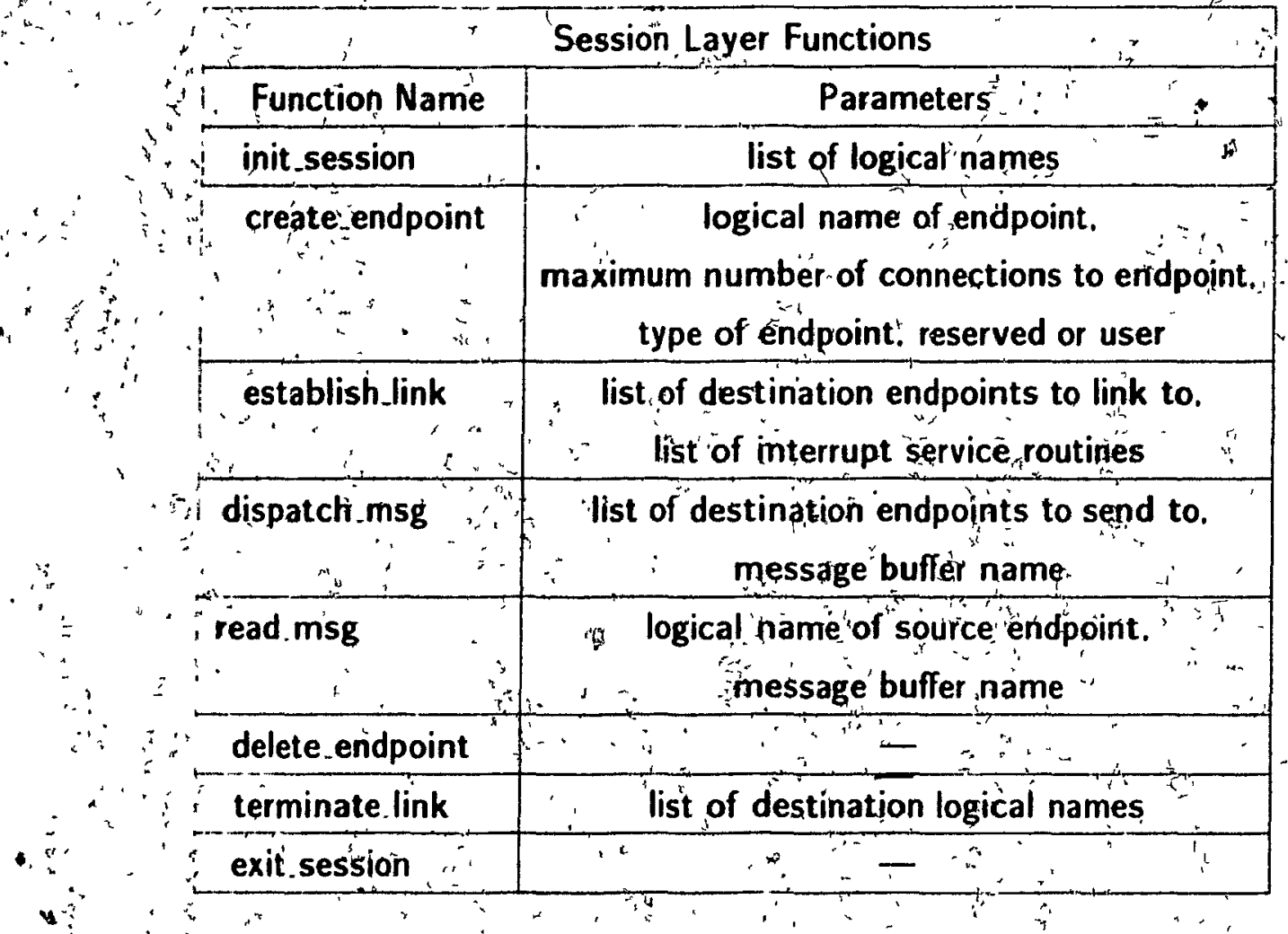

Figure 4.5 The Stssion Layer functions

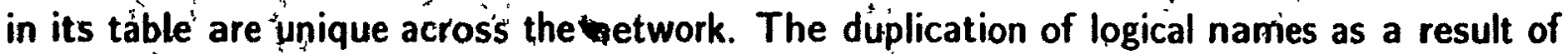
concurrent actions by distinct servers must also be ạvoided. The following prótocol achieves this by establishing a master-slave relationship between the Administrative Servers.

Prior to updàting its table. the Administrative Server broadcasts a message to the'other Servers requesting permission to perform an update. The broadcast is interpreted by the rêmote Servers as a temporary block on table modifications. "The Administrative Server does not proceéd with the update until all other Administrative Servers have acknowledged the request. Provided that the logical names specified by the user are unique. partial entries specifying the logical name and the identifications of both the user and the host are appended to the Administrative Table. The entries are later completed through with the create endpoint call. The modifications are then broadcast to the other servers, the remote tables are updated and the update block is lifted.

Each server has a predefined priority which is jsed to resolve deadlocks. This 
priority is known by all other hosts in the network. Should two or more servers simultaneously broadcast a request for permission to perform an updáté, the servers with the "lower priorities will delay their requests: Administrative Servers receiving table update and init_session requests, while -a block on updating is in effect, buffer the requests until the block has been lifted.

The:protocol is most efficient when several entries are appended to the Administrative Table with a single init_session call. Once a server becomes the master, it can make all of its additions and the Administrative Table update protocol is invoked once. 'Endpoints not ideñified thróugh init session are initialized through create endpoint arid the update protocol must be performed each time create. endpoint is called: this increases the Sessión Layer's initialization time.

\section{- Create_endpoint}
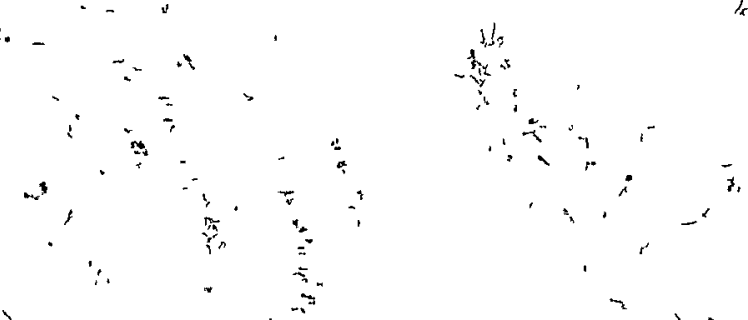

This routine creates a single Session Layer endpoint. The user specifies. the". logical namie of the endpoint. the maximum number of connections that can be accepted at the socket from other endpoints. and the type of endpoint. either reserved or useris

Users may specify permanent Administrative Table entries by declaring an endpoint type of reserved. otherwise it is of type user and mày be maintained throughoùt the life of the process that called its creation. Reserved logical names are intended to be associated with work-cell utility processes sucht as controllable stages. sénsors. or lighting.

- When a process calls create endpoint. a connection is is established to the Administrative Server via a well known socket port and the logical name of the endpoint, is passed to the Administrative Server. The setver in turn. yerifies whether the isser has been, granted the use of the requested logical name through a previously made init session call. This condition is signalled by a partial entry in the Administrative Table, referencing the logical name and the identification of its owner. If partial entry is not present, it is

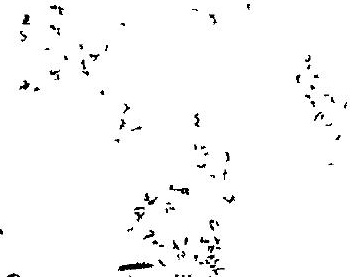


added through the init session table update protocol Each process participating in lipc may créate only a single endpoint.

Once the uniqueness of the logical name has been verified. the Administrative Server creates the requested endpoint and enters all the pertinent information in the Administrative Table. It then creates a Dialogue Server which is dedicated to the newly created endpoint and possesses communication links to both the Administative Server and the user process: The Dialogue Serrver listens at the newly created endpont for connection requests from other Dialogue Sèrvers.

\section{- Establish_link}

This routine establishes communication channels to other processes. Links are connected between the Dialogue Servers associated withe end processes, enabling interprocess communication yia the servers.

The user provides list of logical names of destination endpoints rassociated with 'other: Dialogue Servers. These endpoints must be described in the Administrative Table prior to invoking this function.- The Dialogue Server establishes the required links. and appends their descriptions to the Dílogue Table.

Also passed as a parameter when the function is called is a list of interrupt service routines, one corresponding to each destination endpoint. "A software intèrrupt invokes the appropriate routine when a message is received at the endpoint. Table. whether the link already exists. Should it exist, a duplicate link is not established. Otherwise, an active socket is created and connected to the destination through its associated port. which is retrieved from the Administrative Table by referencing its logical name. For each link established. the Dialogue Table is updated to include the socket descriptor of the new active socket and the logical name of the destination endpoint. The server then 
transmits the logical name of the source endpoint over the newly created link in order.that the destination Dialogue Server mán update its table.

If prögrams áre written by differênt users, it should not be necessary to àssuméne any kind of master/slave relationship requiring that one particular program be responsible 'for establishing the communication links to the other, as would be the case if NIllor the TCP functions were employed. This is significant feature of the Session Layer as it allows programs to be developed indêpendently, without reguard to a particular IPC initializátion scheme: The Session Layer allows either or both communicating programs to be the master. If one program is the master the other will automatically assume the role of the slave. thereby ensuring compatibility. Thexceptions to this are prosesses that are bound to reserved endpoints. They are always slave processes. meant to be utilities that lie dormant. listening for connection requests from processes that call establish link.

Special precautions must be taken to prevent the concurrent actions of two Dialogue Servers from resulting in the dupliçation of línks. When establishing a link between two endpoints, the Dialogue Servers ensurre thatr accidental duplication of the link will be undone. Consider the situation where two Dialogue Servers each attempt to initiate a link to the other at the same time. Neither would find the required connection described in their respeçtive Dialogue Tables and both would proceed to establish the link. The Dialogue Server accepting the connection is required to verify that two similar entries to its table hạve not been made If duplicate entries are present, then one link must be remóved. Tó avoid removing both links. a link is removed if the ASCII equivalent of the logical name of the acrepting endpoint is less than that of the initiating endpoint.

\subsubsection{Management Routine's}

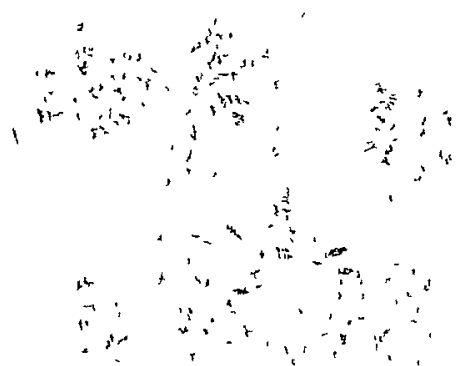

This routine sends a message, passed from the calling process in a character
one or more links to the destination(s) specified in a list of logical names.

- Dispatch_msg 
- Read.msg

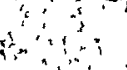

This function call returns the next message received over the link from a specified source. When the Dialogue Server receives a message at a socket. it signals its user process with a software interrupt. A different interrupt is associated with each link? The user process can therefore identify the link over which a message has ben recelved. When the user process receives the interrupt. its interrupt service routine is executed. The contents of the service routine is at the discretion of the user but it generally includes a call to. read.msg. Alternatively. UNAX allows the user, to disable interrupts, in which case any received signals are ignored.

itis 管

Messages sent or received over any link are appended to its endpònt's associated send or receive FifO buffer, which is approximately 2 kilobytes in length. iuntil transmitied or read. Because of TCP s, inherent reliability. messages are not lost if the endpồnt 's buffer becomes full: further transmissions to that sócket are blocked until sufficient space becomes available

\subsubsection{Termination Routines}

\section{Deléte_endpoint}
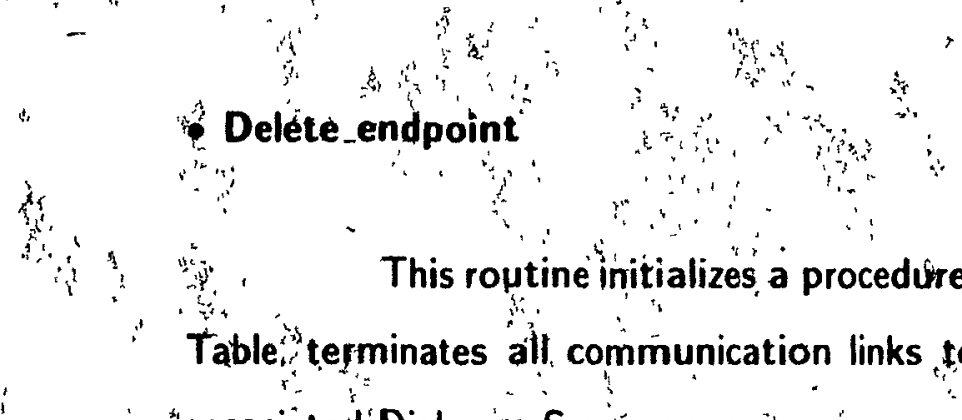

This routine initializes a procedure that deletes an entry from the Administration. Table, terminates all communication links to the specified endpoint. and terminates the "associäted Dialogựe Servèr proceşs.

Prior to closing a connected socket, the Dialogue Server notifies each destination endpoint's Dialogue Server of the impending termination so that the destination endpoint may be closed and the link's entry may be removed from all the Dialogue Tables." The Dialogue Server instructs its Administrative Server, to delete the endpoint entry from its table and to inform other Administrative Servers of the table update. The socket is then closed and the associated Dialogue Server process is terminated. 


\section{- Terminate لlink}

This function call terminates communication links and remóves their descriptions from the Dialogue Table.. The links are identified by the logical names of their 'destinations. The Dialögúe Server notifies the destinations' Dialogue 'Servers of its intent' to terminate the links so that they can update their Dialogiue Tables: The sockets supporting the specified link's are then closed. UNIX enforces a limit on the number of files that maý be open to a user at any one time. "The number of processes per user and the number of open files per process are set to 25 and 20 respectivèly by default in a standầid UNIX 4.2BSUiconfiguration. Endpoints look like open files to the operating system and in applications where the number of endpoints and open files approaches the user's limit, it is conceivable that infrequently used links would be opened and closed when they are needed.

\section{- Exit_session}

This routine ends the session by clösing any open sockets in an orderly way and terminating the user process.

\subsubsection{A Sample Session Laýer Program.}

The following example illustrates an implementation of some of the Session. Layer's functions. Two short programs are presented whose objective is to establish the :simple but commonly used architecture depicted in Figugre 4.6, and to cycle a message through each: proceșs.

\section{,}

The first program is composed of a single process. - which is identified as process A. The second program is made up of a two processes: a parent process andachild process. identified as processes $B$, and $C$. respectively.

Prior to executing these programs, the Session Layer must have first been invoked on one host. A boot-strap loading procedure then invokes the Session Eayer 



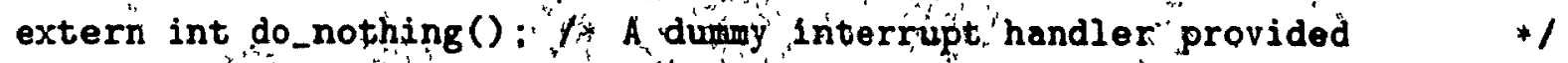
ant
1* by 'the session layer
int
1* A global flag
$* 1$
char mǵgibUFFER_SIZE]
1* Array declaration for the message
get_mig_c (sigño)
in't
pigna,
$\{$ read_msg ( $\mathrm{C}$ ' , msg) done=1; $\}$
Y Interrupt service routirie invoked when $a: * j$ I* message artives fróphrocess C's endpoint. *I
I* The fuhction reads the messag into meg */ /* and sedts the global variable done

matn ()$^{2}$

$f$ int get_msg_c( $)$;

int_fnzs,

fn[0] do nothing:

$f n[1]=g e t \_m s g \_c$;

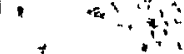

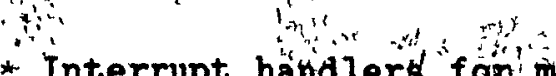

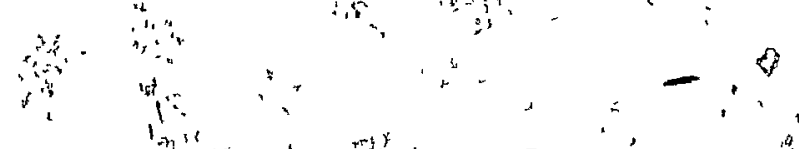

1* at a from endpoints"B and C respectively

if $(f \operatorname{rork}()==(0) \quad$ Remotely invoke process $B$ on hơpt $B$

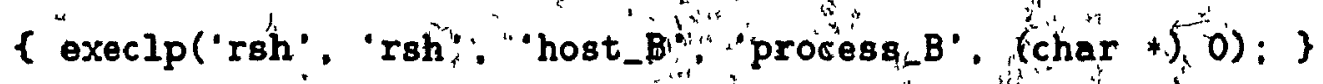

init_session $\left(A^{\prime}\right)$; $\quad \because / *$ pass the admin. 'server thondpoint list *l

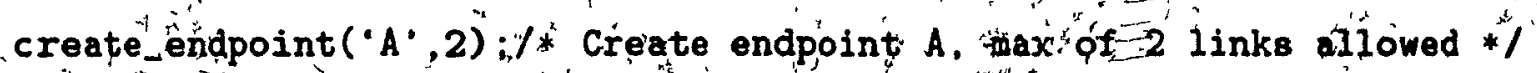
estäblish_link

('B,C',fn):

/* Establish links to endpoints b and C

dïspatch_msg

('B': $\left.\left.A^{\prime} \cdot\right)^{\prime}\right)$;

/* Send the messages " $A$ " to, the endpolnt $B$. *I

while (!done);

/* wait" loop. Done is set to 1 by get_mbg, $C_{F} ; 1$

strcat (msg. $\left.A^{\prime}\right)$ :

1* Append the process name A to the message */

printf ('In\%s\n',msg): /* Display the final transmission: A B C A */

terminate_link ('B,C'): $/ *$ Terminate links to $A$, this is optiona $*^{*}, *$ "

exit_session $(0) ;\} \quad 1 /$ * closes any open file descriptors and exit * 

\{ create_endpoint

4

\section{in $[0]=$ do nothing:} fn $[1]=$ get_-msg-B; establdsh_link

$(A, B \cdot(f):$

whìle (!donet): sircat $(\dot{m a g}, \quad C \cdot)$ dispatch $>$ msig

( 'A'. ms'g) : exit_session $(0)$;
1* Create endpoint $\mathrm{C}$, max of 2 links allowed /

/* Interrupthandlers for messages recéd vad $* /$ I* at $C$ from endpoints $A$ and $B$ respectiyely
$+1$

/*: Wait loop until global done flag is dot " */ $f^{*}$ Append ' $c$; to the message being cycled

1. Displatch the message to process A (1) 1* closes any open flo descriptors and exit */ /* End of process C

\} sing establish_link

$$
(\text { ( A C., }, \mathrm{fn}) \text { : }
$$

/* establish links to $A$ and $C$ irom endpoint $B * /$ while (ldone):

I* Wait loop until global dóne flag is diet strcat" (m8'g, 'B') :

f* Append : Bi to the messag

\subsubsection{Additional Features of the ISO Session Layer Model}

With eaç refinement of the Basic Reference Model. the: Session Layer fras become more complicated. Session tayer concepts have evolved from a concept [Zimmermann 80] to a well defined protacol [Emmons and Chandler 83]. In part. this reflects the growing 
44 The Śssion Layer Design maturity of protocol research as weth as' a better understanding of the services needed by the Application and Presentation Layeis.

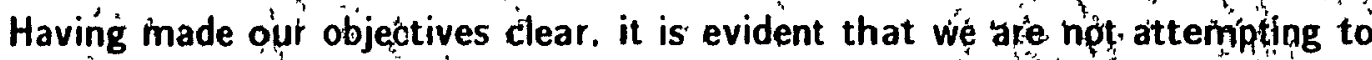
fully implement the OSI Session Layer Model. Instead, the deșign is tailored to satisfy" the needs of our robotic environment: At this time, we would hike to briefly mention some of the features of the ISO's Basic Reference. Nodel that are not included in this implementation

$\therefore$ Quarantise function that allows ap entity to send and receive messages in groups. instead of individually. This is intended primarily for transaction-ariented activities such as those assöciated with database management. The furrent design allows the user to append sseveral messages and transmit them all to thame destiration. Future implementations could allow for grouping messages that are bound for different deșinations.

- Expedited Data. Exchange function that circumvents the flow contripl imposed on normal messages. This would typically be used for smalt high priority messages such as aboritt. This is certainly important: and will likely be added in a tater revision: This service would require the support of an expedite feature the Transport Layer.

- Synchronization function that allows a user ejitity, to specify whether or not an acknowledgement should be provided by the siturver after each successful transmission. Because TCP guarantees message, defivery there, is no need for this feature. except " perhaps as a way to synchrónize twộter processes. In effect. the Dialogue Servers indirectly provide thịs synchronization by arrives.; The user can therefore implement synchronization through the proper use of the Sessiọn Láyer function calls.

- Resynchropization function fó trạ́nspărènt crash reccovery, via "róllback to" a "checkpoint'. This is useful when sending large anounts of data from a single entity. The 
dialogue server would perióndically mark a position in the data stream and re-transmit from that mark. This would represent a much larger server overhead, as extra buffering would be required within the Session Layer. Since IAN Transport Layer service is very reliable. the overhead incurred in implementing this feature is judged to be unnecessay at the present time.

- Dialogue Coñtrol fưnction to permit eịther full duplex or half duplex communication over the links. Since TCP supports full duplex communication, it is unnecessary to offèr thése alternátives.

- Haindshake on Termination function which ensures that both communicating entities have no mơre messages' to exchange and all data in transit is delivered prior to termination of the connection. This feature is more appropriate in 'long-haul networks where messages move slowily. In our LAN environment, the propagation delay is' relatively small. Handshaking is left for the user to implement.

- Afcess Control function which restricts communication access. The UNIX operating system inherently grants us a limited form of access control by virtue of the owner. group. and world permission attributes associated with, files. We feel that this is sufficient at the present time: Future implementations may allow access to an endpoint based on user identification numbers.

\subsection{Network Interface Library-(NIt) Design}

\subsubsection{Q'verview}

The inter
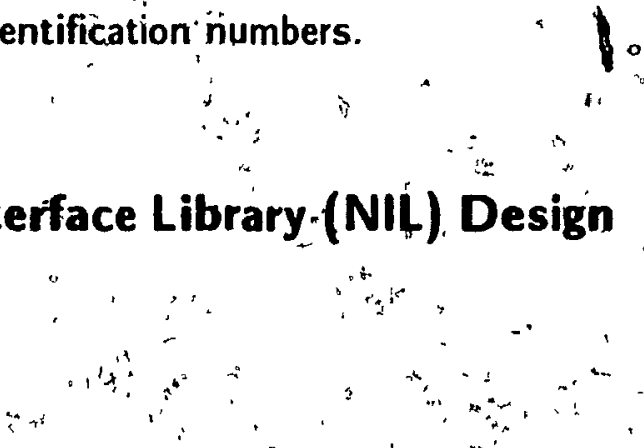

The interprocess communication network interface library (NIL) is a high level library of $\mathrm{C}$ functionns which fácilitates communication between programs executing on one or more hosts under the UNIX 4.2BS operating system." The library functions simplify the implępentitation of IPC appliçations by $\mathrm{P}^{2}$,iding an easy interface to the system network as

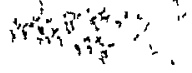


45 Network Interface Library (NIL) Design,

well as building blocks necessary to implement some of the more commonly used communication' architectures. The basic services provided allow the creation of socket endpoints. the establishment of links between the sockets: the sending and receiving of messages over the links. and the termination of the links and sockets:

In terms of its degree of sophistication. NIL lies somewhere between the Session Layer and the low level TCP interface functions próvided by UNIX. The functions contained in the NIL library are built upon the TCP functions. "Programs implemented with either NIL or the TCP functions would be expected to execute at approximately the same speed since the NIL functions aggregate the TCP and other necessary UNIX functions into a form which is convenient for our applications.

In comparison to the Session Layer. NIL based communication is faster because "it doesn't have its IPC mèdiated by servers: Links are established directly between user processes within the same or across different host boundaries. Messages therefore are transported over a single link instead of three as. in the case of the Session liayer. The NIL functions parallel those of the Session Layer in their application; if a user understands how to use the Session Layer, he will be able to easily understand the NIL functions.

3

A disadvantage of using NIL over the Session Layer is its relative lack of power. The user has to do móre work. writing longer programs to obtain a comparable implementation. The functions are also harder to use because some understanding of the TCP network fujctions is necessary.

if

The most important disadvantage is that the modular. standardized environment provided by the Session Layer is not present. An important concept of the Session Layer is to provide a high. level. standardized environment that would facilitate integration of work-cell processes written by different users. Ekentually a libtary of work-cell utility programs or modules would be accumulated that could be used by any member of the laboratory in their experiments. NIL does not provide any form of standardization. Because of this. the IPC architectures must be specified at the time that the communicating programs $r$ 
45 "Network Intertâce Library. (NIL) Design.

are designed. NIL is best suited to simple applications where a "small number (one or two) of fast communication links are involvedd and the architecture of all participating programs is well defined. Without standardizâtion. each jindividual must maintain his own set of work-cell utilities. which is counterproductive to the laboratory as a whole.

A sessión can simultaneously incorporate communication structures from either the Session Layer. NIL, or TCP: one type of interface does not preclude the pthers can be, used to great advantage by a knowledgeable programmer when the work-cell timing is criticial.

\subsubsection{The Network Interfáce Library Functions}

As in the case of the Session Layer, the Nit functions can also be divided into : three classes: initialization routines, management routines, and termination routines. The NIL"functions and their associated parameters are presented in Figuress 4.9. 4.13, and 4.14. A set of sample NIL programs. which implements the same example presentedearlier that was implemented with thession Layer.

\subsubsection{1 - N!L.Initialization Routines<smiles>[C]1[CH]CC#CC1</smiles>

The NILjinitialization routines are summarized in Figure 4.9.

\section{- Session_init}

- Session_server jinit
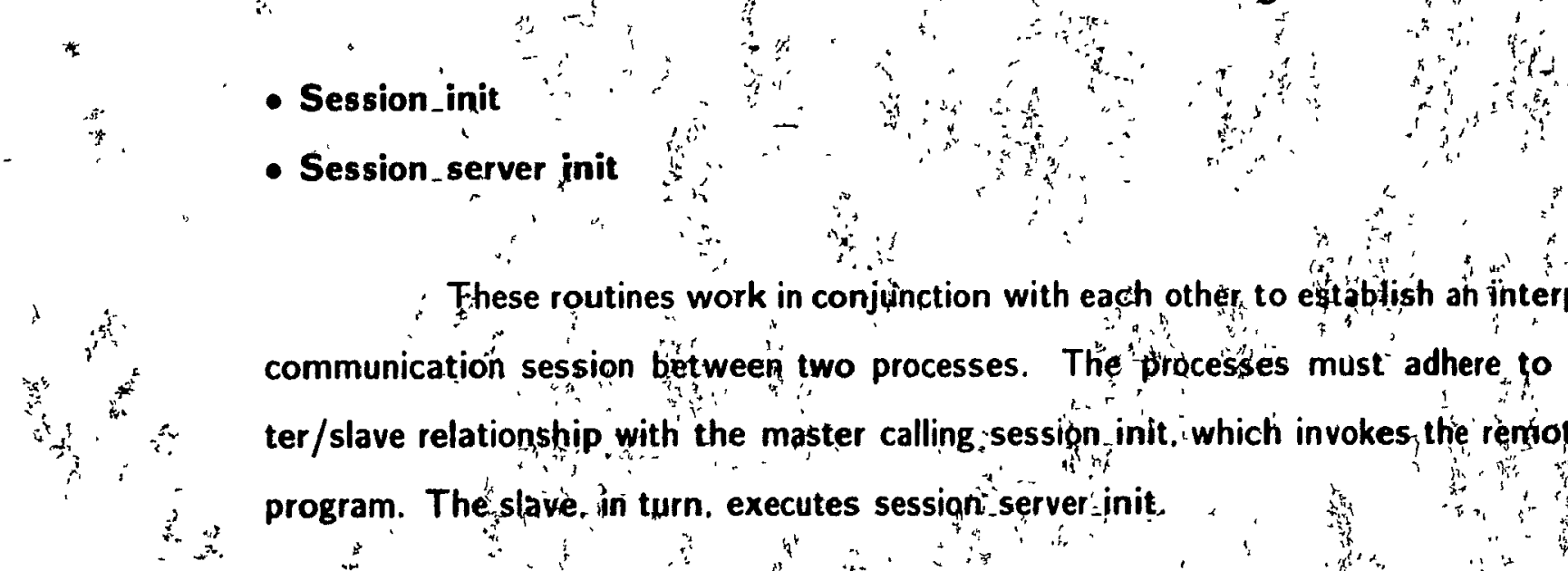

These routines work in conjuinction with each other to eqtablish ah interptocess communication session between two processes. The processes must adhere to a nas ter/slave relationship. with the master calling,session init, which invokes the reniote slave program. The slavè in turn, executes sessigniserver init.

- Passive_socket
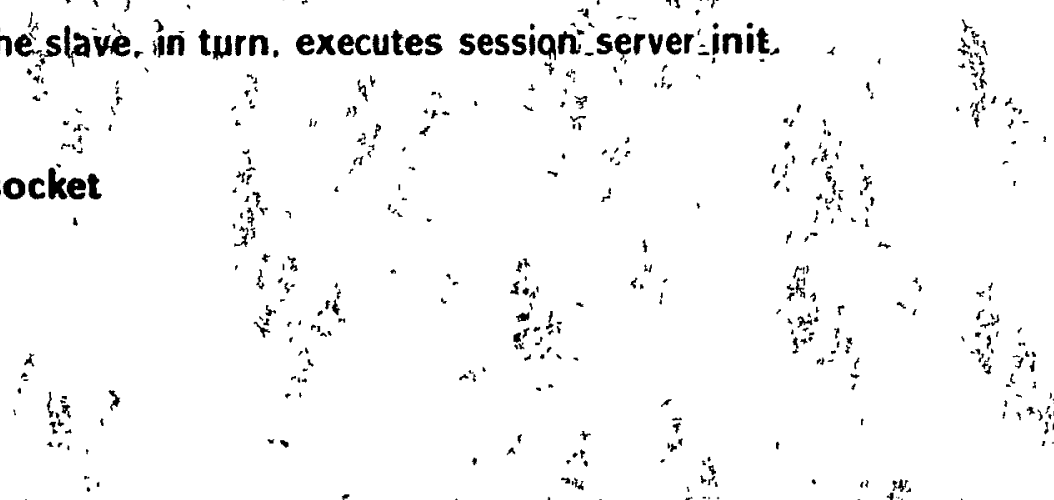
Network-Interface Library Initialization Functions

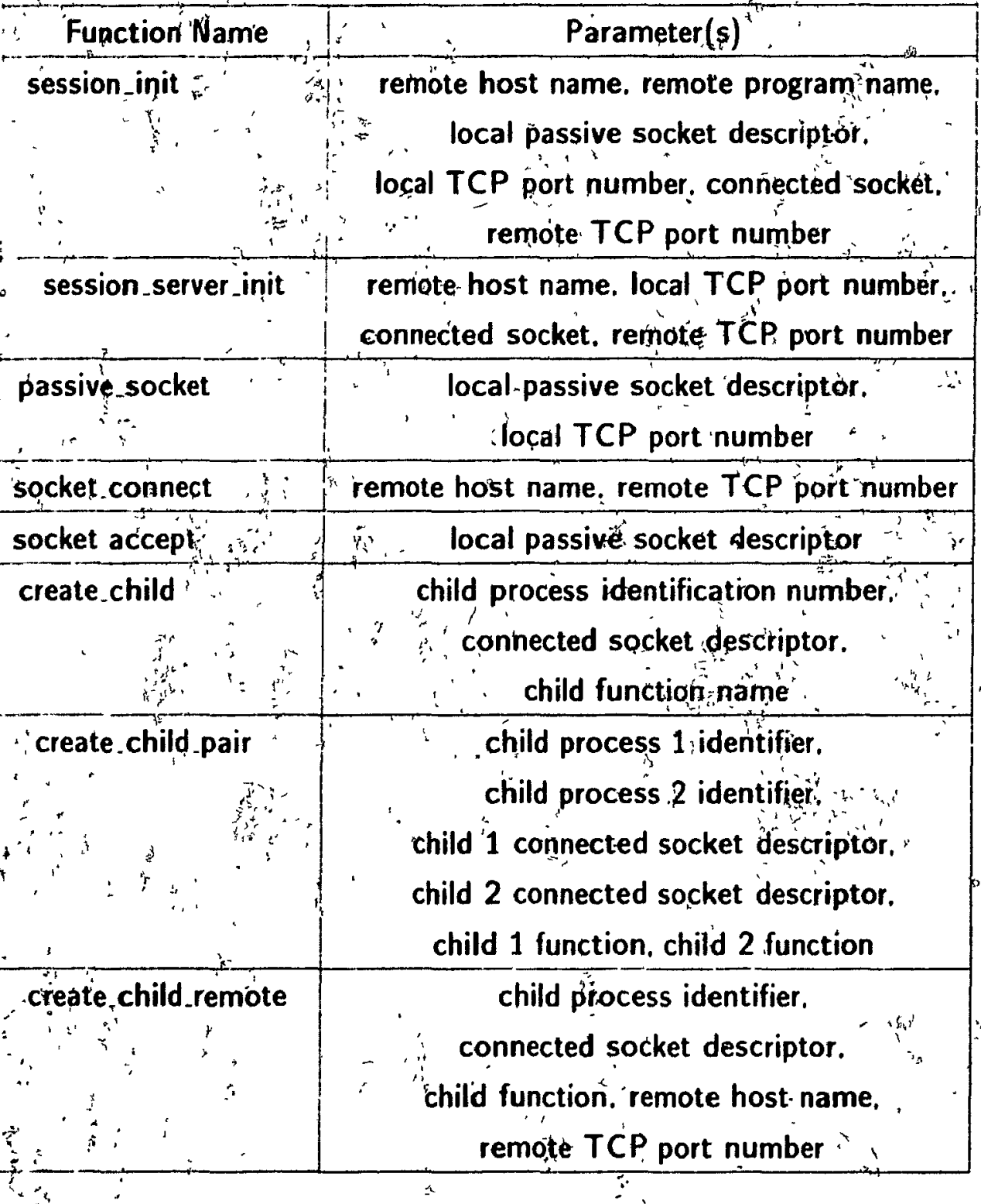

Figure 4.9 Network Inteŕface Library inị̂ialization functions

政 (3)

The function passive_socket establishes a communication endpoiñt which may accept connections from other endpoints, that is, a socket which has been bound to a TCP

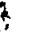
port. Following the call, the newly created passive socket listens for cannection requests from active sockets. The TCP port number, which is assigned by the operating system, and the socket descriptor, are returned ta the user. The socket descriptor must be refenced Ky the user when accepting connections to the endpoint, while the TCP port number must 部?

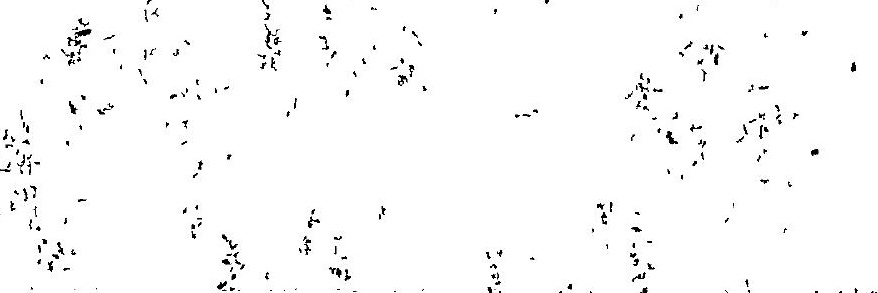


be supplied to any process planning to establish a cońnection to the endpoint.

\section{Socket_connect}

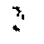

The function socket connect creates a socket and connects it to the passive socket host which has been bound to the specified TCP port on the identified host. The function returns the descriptor of the connected socket, which misst be referenced when; sending or receiving messages.

\section{Socket_accept;}

This routine socket_accept is used to accept à connection at a specified socket. "The accepting socket must be passive and listening for connection requests. When connection is accepted, a copy of the original passive socket is created and connected: the original' socket remains open and may continue to listén for and accept additional connections. The descriptor of the connected copy is returned to the user, to be referenced when sending or receiving messages over the link.

\section{- Create_child}

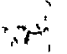

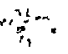

This routine forks ${ }^{1}$ and establishes a communication link between the parent and child. The child process executes' a specified function. passing as a parameter the socket

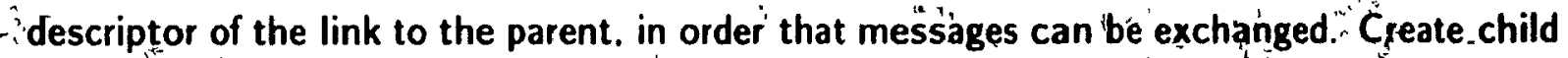
returns the process identification of the child process as well as the socket descriptor. to be referenced by the parent when communicating with the child. The communication

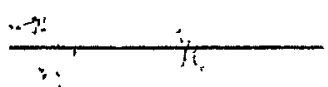

1 A UNIX fork splits a program, into two concurrently executing copiés The original program is called the parent and the copy is called the child Each propess forks its execution, path. with the parent proceeding in one direction and the child in the.other, that is. the child executes one part of the original program and the parent executes the rest.

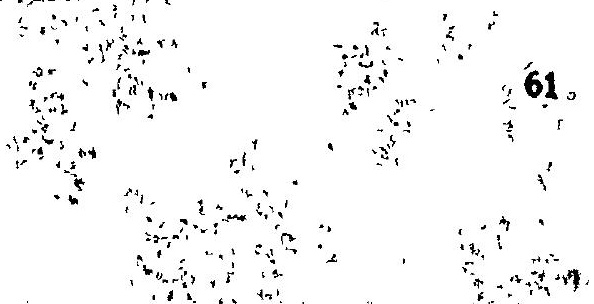


45 Network Interface tibirary (NIL) Desjgn

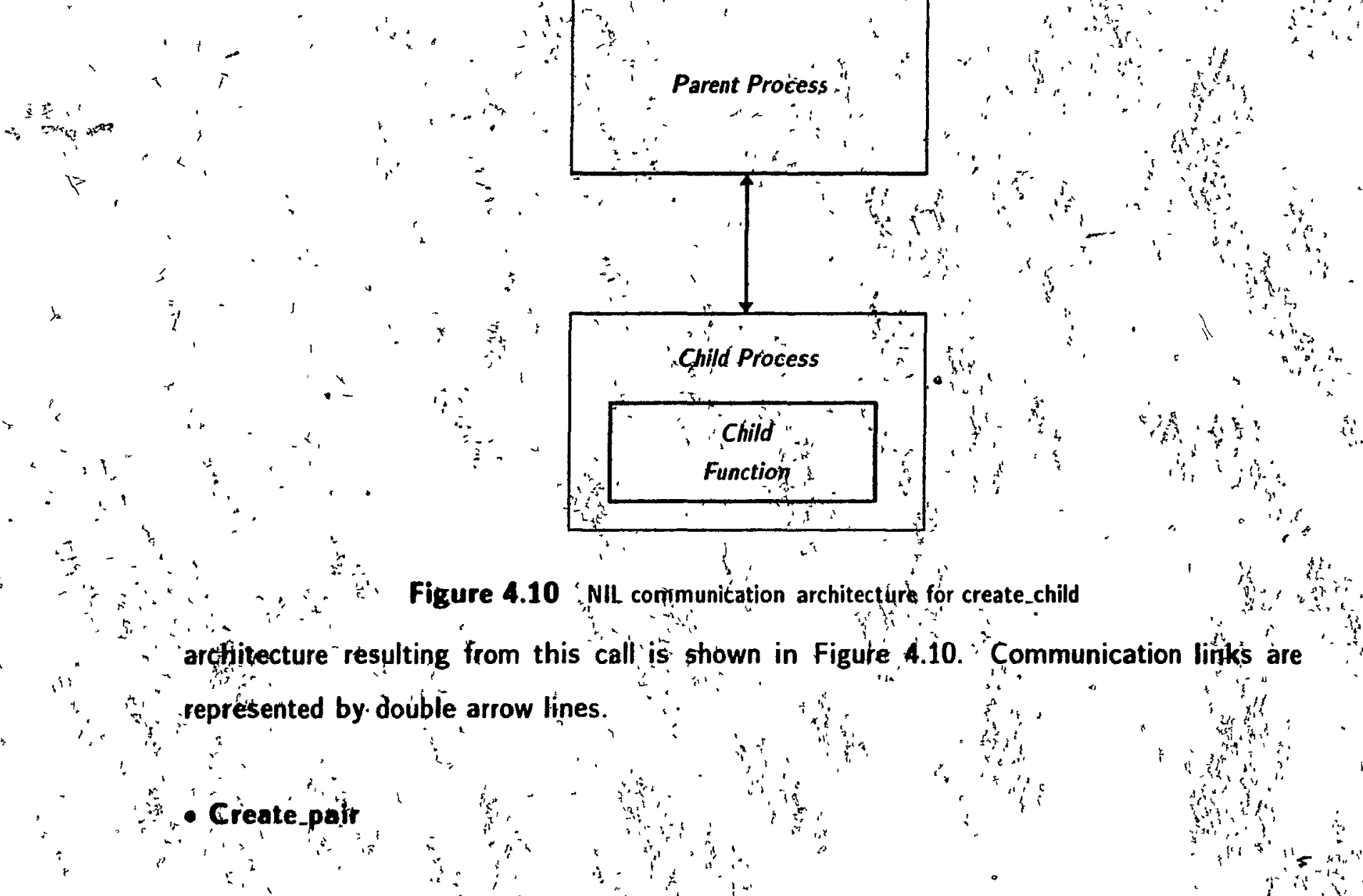

This function forks a pair of child processes, with socket connections estab " lished between the parent and each child and betwen the two children. Each child process"s executes a specified function, passing as parameters the descriptors of its socket connections to the parent and to the other child. Create_pair returns the pracessidentification of the child procèsses as well as the socket descriptors which-must be referenced by the parent when communicating with each child. 'The communication architecture resulting from this call is shown in Figure 4.11.

- Createschild_remote

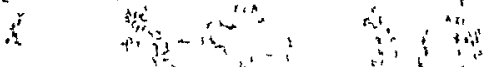

A child process! is forked, with socket connections established between the parent and child, and between the child and a remote port. The child process executes a specified function, passing as parameters the descriptors of its two socket connections Prior: 
4.5 Network Interface Library (NIL) Design

1

0

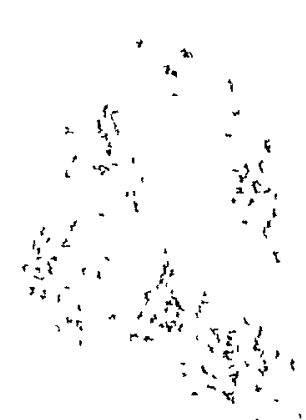

20

然

20

等 i.

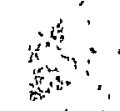
1
$\vdots$
$\vdots$
$\vdots$
$\vdots$ $f$

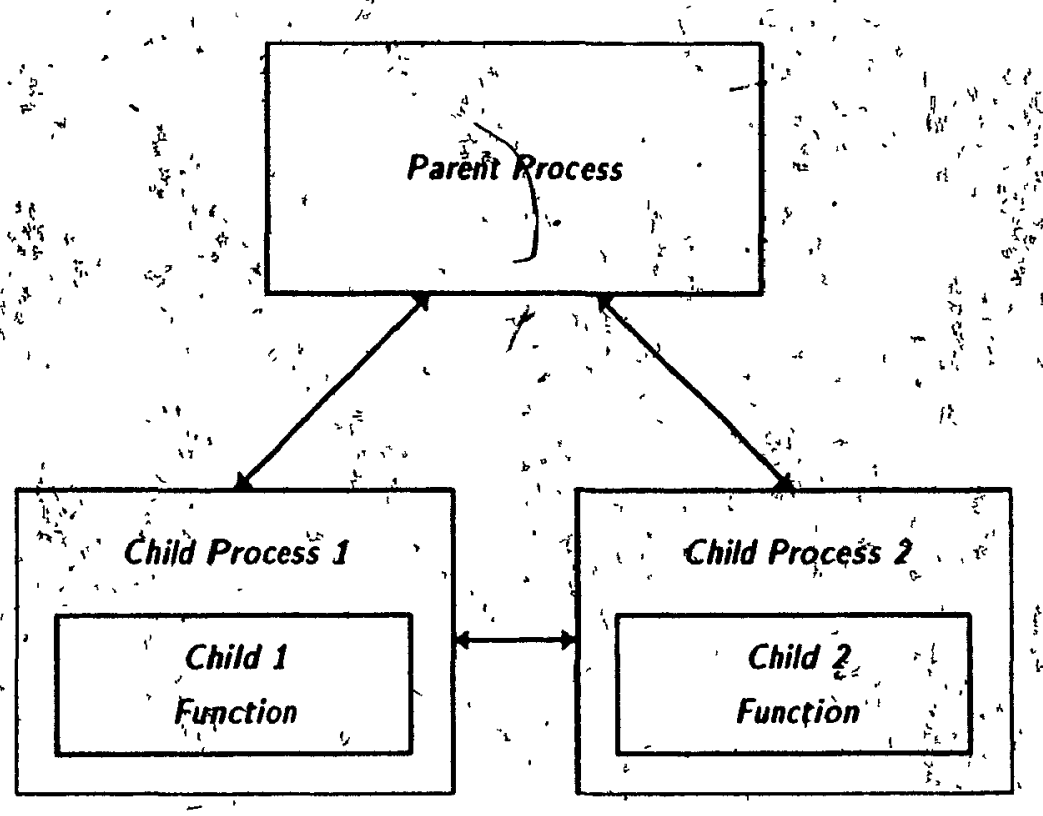

Figure 4.11 NIL communication architecture for crẹate.pair

to calling this function. the parent process must have executèd session:init: the remote process must have executed session server_init and socket accept "The communication architecture resulting from this call is shown in Figure 4.12.

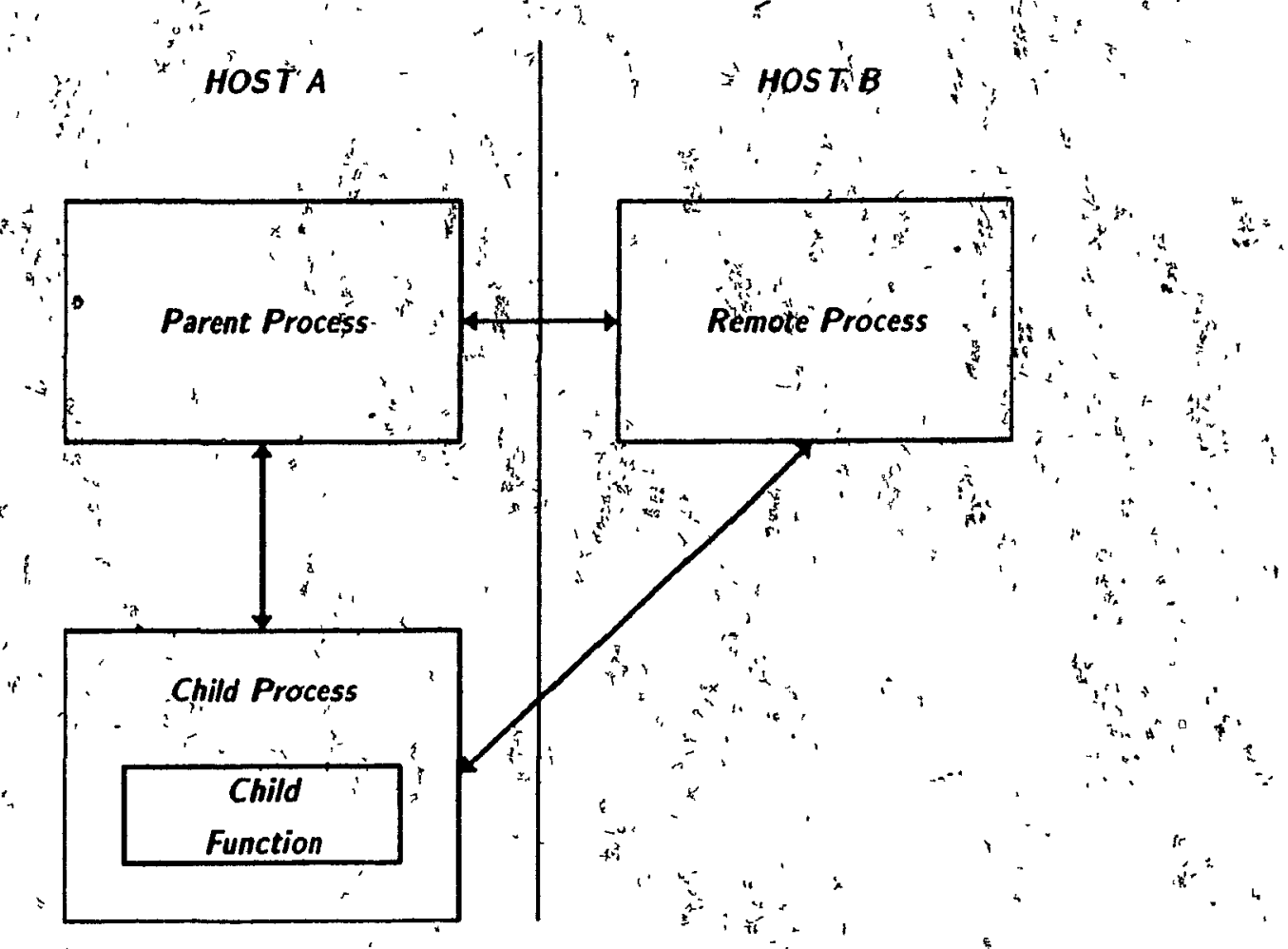

Figure 4.12 NIL communication architecture for create_child remote 
44 Network Loterface Library (NIL) Design

- Send_msg

- Sendint

Send char

These three routines tranșmit a character string. integer, or single character variable over the specified links.

\subsubsection{NIL Termination Routine}
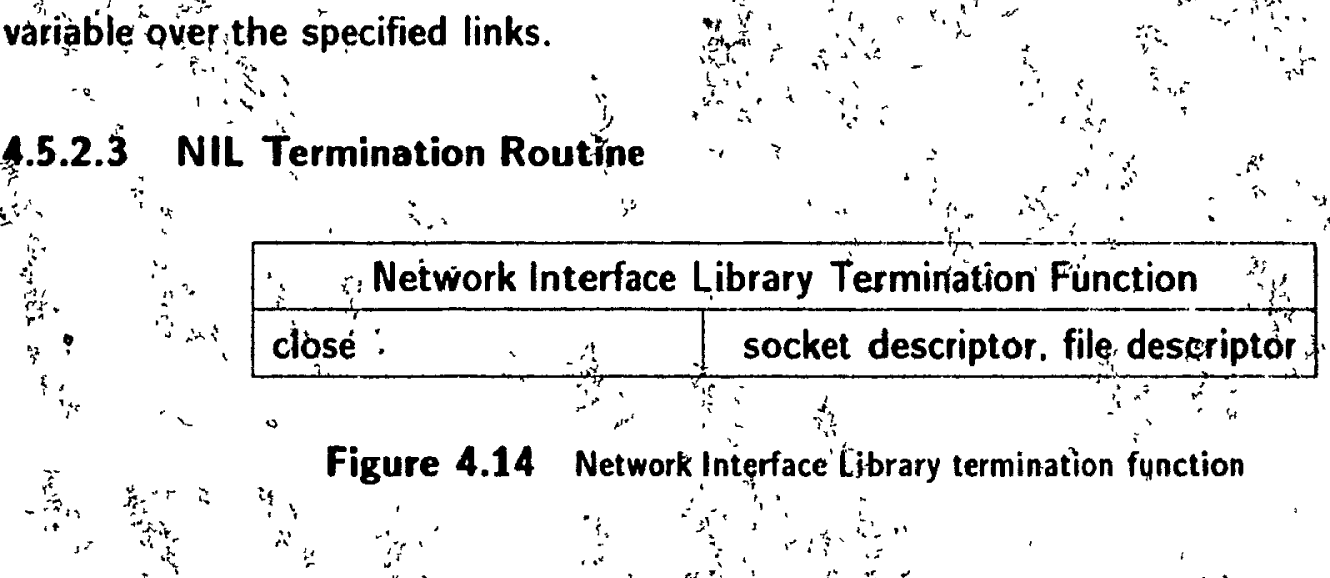

Figure 4.14 Network Interface Cibrary termination function

... The NIL têrmination routine shown in Figure 4.14.

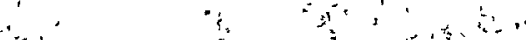

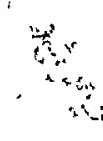

\section{This routine is standard UNIX function which is used to close an open socket} of file descriptor. It is considered a good programming practice to clóse any sockets or files. which were opened by the user. prior to exiting a program.

\subsubsection{A Network Sample Interface Library Program}

The following example illustrates an implementation of some of the NIL functions. Two programs, whose objective and communication architecture are identical to the

Session Layer example presented in Section 4.4.5. have been implemented. The first program is again composed of a single process, identified as Process $A$ : the second program is 'made up of a two processes: a parent process and its child. identified as processes B and $C$. respectively. 
'Initialization of the session is achieved through a master/slave relationship between the two programs: process $A$ remotely invokes process $B$ and initiates a communication link to it, through a call to session_init. Proces calls session_server_init to complete the establishment of the communication link. Process $C$ is created by forking process $B$ and is linked to both its parent procesś and process $A$. through the function create_one_remote. Using send_msg and recv_msg., a message is cycled from process $A$ to $B$ to $C$. and finally returned to $A$ and displayed at the user's terminal.

As in the previous example, comments have been included to assist in understanding the implementation.

Figure 4.15 NIL Application Program 1 - Process A

"include "NILconfig.h" \#include 'NILdef $8 . h$ '

$\operatorname{main}()$

\{ char msg[BUFFER_SIZE] ; int session_server_init

( 'host_B'. 'proce88 $\_$B' , Lpassive_soc_A. atcp_port_A. 'sooc_B. $\lambda$

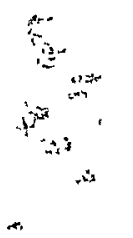

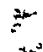

और "in (

4 2.

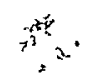

tcp_port_A. passive_80C_A. tcP_port_B. soc_B. $80 c_{-} \mathrm{C}$; ! $x^{2}$
/* NIL host configuration header file */ i* NIL constants and definition . */ 


\section{dtcP_port_B )}

4.5 Network Interface Library (NIL) Design

Q $=\lim _{0 \rightarrow \infty}$

BoóC = socket_açept

( passive_soc_A'):

I: Accept a. connection request from

strcpy (msg. $\left.\cdot A^{\prime}\right)$;

send_msg (soc_B.msg): of* Send a meserge containing the process* $f$

I* name, A, to process B while (recv_msg

$\left.\left(\operatorname{soc}_{-} C, \operatorname{msg}, 10\right)==0\right)$; ,
I* Read a message from the link soc_C :*/ $y_{*}$ into the buffer $\mathrm{msg}$ with a timeout *J. is of $10 \mathrm{sec}$

strcat (msg. ' $A^{\circ}$ );, $1 / *$ Append pròcess namé, to the message */ printf $(\cdot \ln \% \mathrm{~s} \backslash \mathrm{n} \cdot \mathrm{msg})$; /* Display the final message: A B $\bar{C} . A * /$ close (passive_soc_A): $\quad \because / *$ olose open socket descriptors? close (soc_B) : close (soc_C) : $\operatorname{exit}(0)$;

\section{fond exit}

Figure 4.16 ' NIL Ápplication Prógram 2 - Processes, B ánd C

\section{*include 'NILcónfig.h' \\ *include 'NIĹdéts.h'}

'/* NIL "configuration heádér file

$\ln -\mathrm{N}$

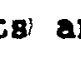

and definitions

\section{$\operatorname{main}(\operatorname{argc}, \operatorname{argv})$}

int arge:

çar **argvi

$\mathbf{0}$

$\cdots+$ [ char m8g[BUFFER_SIZE] 


$$
\begin{aligned}
& \text { int tcp_port_B? } \\
& \begin{array}{c}
\text { passive_soc_B. } \\
\text { tcp_port_A, } \\
\text { soc_A. } \\
\text { fnc_chjld_c(). } \\
\text {-soc_child_c. } \\
\text { child_c_id: }
\end{array} \\
& \begin{array}{c}
\text { passive_soc_B. } \\
\text { tcp_port_A, } \\
\text { soc_A. } \\
\text { fnc_chjld_c(). } \\
\text {-soc_child_c. } \\
\text { child_c_id: }
\end{array} \\
& \begin{array}{c}
\text { passive_soc_B. } \\
\text { tcp_port_A, } \\
\text { soc_A. } \\
\text { fnc_chjld_c(). } \\
\text {-soc_child_c. } \\
\text { child_c_id: }
\end{array} \\
& \begin{array}{c}
\text { passive_soc_B. } \\
\text { tcp_port_A, } \\
\text { soc_A. } \\
\text { fnc_chjld_c(). } \\
\text {-soc_child_c. } \\
\text { child_c_id: }
\end{array} \\
& \begin{array}{c}
\text { passive_soc_B. } \\
\text { tcp_port_A, } \\
\text { soc_A. } \\
\text { fnc_chjld_c(). } \\
\text {-soc_child_c. } \\
\text { child_c_id: }
\end{array} \\
& \begin{array}{c}
\text { passive_soc_B. } \\
\text { tcp_port_A, } \\
\text { soc_A. } \\
\text { fnc_chjld_c(). } \\
\text {-soc_child_c. } \\
\text { child_c_id: }
\end{array}
\end{aligned}
$$

session_server_initt

( argv[1] :

argv [2] . uptsotve_80C_B.

$=\infty$

$\sin$ atcp_port_B.

\&soc_A. dtcp_port__.'):

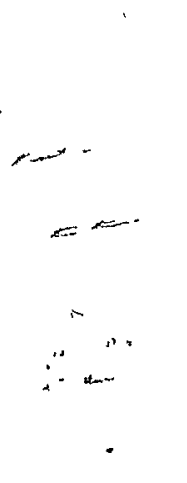

$/ *$ the TCP port of B's endpoint

/* socket descriptor of the endpoint */

/* TCP port of endpoint of process $A * /$

$I_{+}^{*}$ descriptor of link to process A ,

/* function executed by child process*/

/* descriptor of link to child.

$* y$

/* prócess identification of child */

/* establish the communication session $* /$

f* with the master process A. The hosit *

$\because \quad / *$ name and TCP port number of the

I* endpoint owned by process $A$ are

*1

'/* specified by passed parameters"

$*$

/* argv[1] and argv[2] respectively. */

Q

create_óne_romote

$\therefore$ (echild_c_id. 480c_child_c: fnc_child_c.atgv[1]. $\int_{i \rightarrow \infty}^{\operatorname{argv}[2]):}$

while (recvingg $\left.\left(\operatorname{soc}_{-} A, m 8 g, 10\right)==0\right):$ $x=-\frac{1}{2}$ etrcat (msg. * B') :
/* fork a child process. process C, to */ /* be linked to both the parent and the *'/ /* remote process A. The child process * * $f^{*}$, will execute fnc_child_c.
1* Read a message from the link sóc:A *f i* into the buffer m8g with a 10 " second $* A$, $=/ *$ timeout period.

/* Append the process name B to msg
$* /$

*J

"aend_msg (soc_child_c.msg); /* Send the mod̂tied message to the 
46 Comparision of the Sessioin tiand and

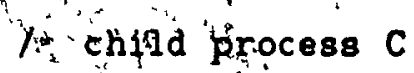

$n^{n}, \cdots$

$$
\begin{aligned}
& =3+ \\
& \text { ind } \\
& \{\because\}\}
\end{aligned}
$$

(This routine is executed by process $C$, the child of process if after the fork"is cálied. Descriptors of socket "link to the

1* parent. process $B$, and to a remote process. A. are pagegd i

$I^{*}$ sociparent_B and soc_remote_ $A$. respectively.

fnc_child_c: (soc_parent_B', soc_remoté_A) jint soc_parent_B, , soc_remotê_

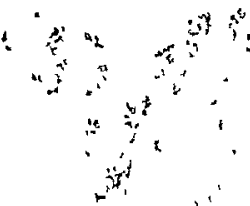

\{ abarag mog [BUFER_SIZE] :

$l^{*}$, buffer for message tratrefer

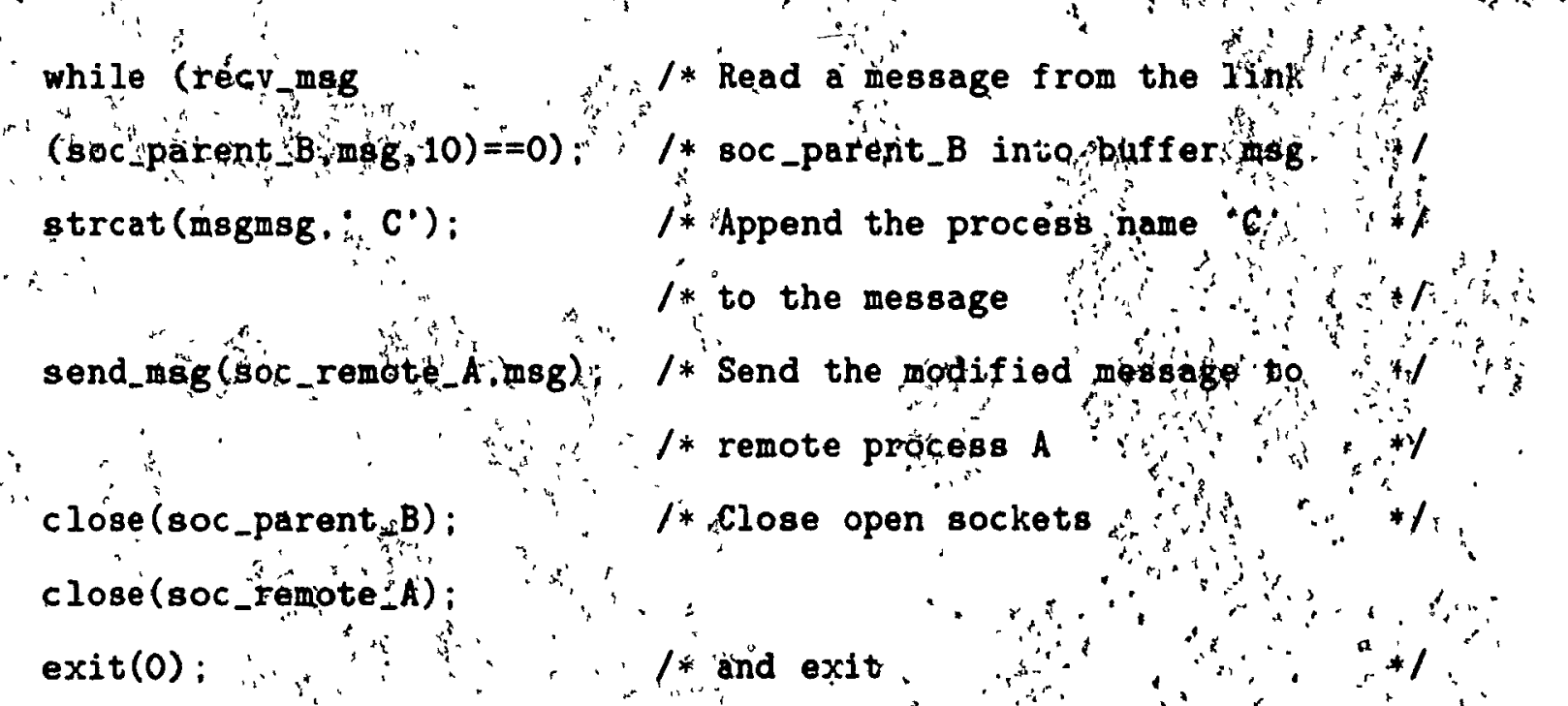

\subsection{Comparision of the Session Layer and NIL}



As a second example, assume thăt a host crashes. If a Dialogue Server attempts to establish a link to a' endpoint on the affected host.. or attempts to send or "receive over a disconnected link, an error is returned from TFP. The Dialogue Server informs the Administrative Server that a link or endpoiht is disabled-The Administrative Server verifies whether the remote host is operational. If not, an approprialte message is broadcast to the other hosts, which in turn, removes entries of endpoints which resided on the affected host ; from their Administrative Tables, Dialogue Servers are notified to remove from their tables all links to endpoints on the affected host.

$\because$
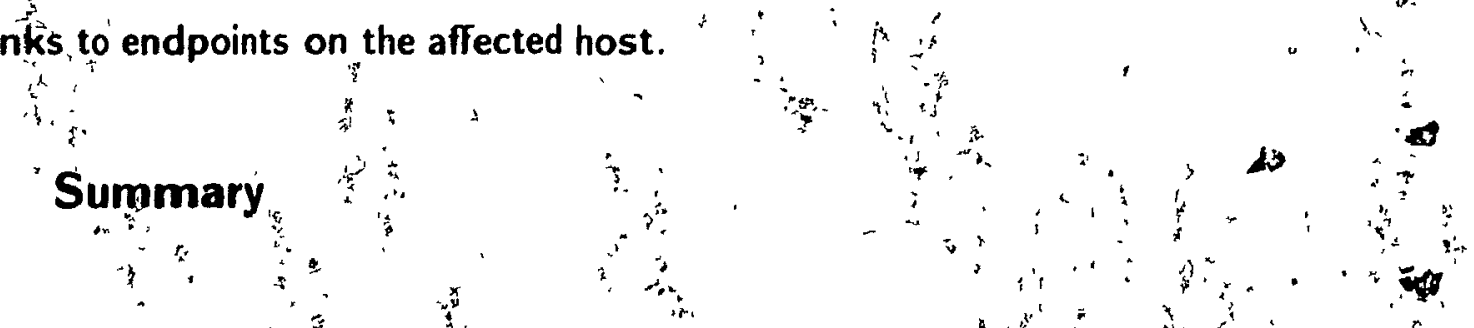

In this chap̂ter, we.have prẽsented the designn strattegy architéctụre, and programming interface of a system for interprocess communication within: ouj distributed robotics environment. The environment is based on host computer sỳstems operating un. der the UNIX 4.2BSD operating system cómmunicating over an Ethernet local area network.

The communication environment is designed to be flexible, to meet the needs of a constantly changing research environment; modular, to support robot work-cells that are composed of distinet elements: and easy to use, so that programmers do not have to devotét time towards understanding the detailed mechanisms of interprocess communjcation.

The network interface is designed to provide a standardized way of achieving 3 IPC while relieving the user from the low level aspects of network communication. Three interfaces are available, each providing a different level of söphistication. Each level provides mechanisms for establishing. managing, and terminating communication sessions, but in a different way. The higher the level. the easier it is to implement and manage interprocess communication in an efficient manner. The lower levels offer increased.speed and flexibility

The highest level interface is based on a partial implementation of the ISO's Session Layer service specifications. The implementation is based on a hierarchical framework composed of an Administrative Server which is responsible for maintaining communication 
"x a de endpoints afd a Dialogue server which is responsible for managing the communication links: Of the three interfaces; this is the only level that offers a standardized way of achieving IPC.

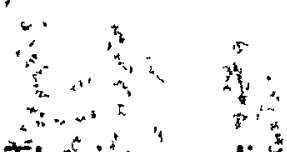

The intermediate level interfacte is provided by the Network Interface Library.

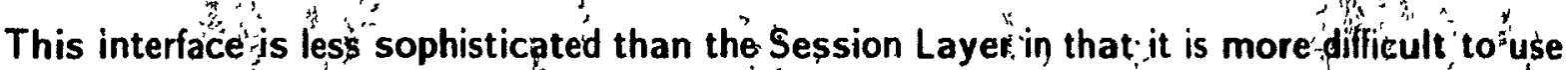
and its functions are less powerful.

The lowest level interface is provided by UNIX 4.2BSD the form of its TCP function library. This level offers the most flexibility and speed but these attributes are offset by the difficulty encountered in using the TCunctions. This level is primarily of interest to system level progitammers.

Two sample.programs illustrating both the Session Layer and Network Interi face Library functions were bresented to demonstrate the ease with which interpiocess" communication can be effectéd.

Error handling capabilities of bọth the Session Laye and NIL initerfaces was presented. The Session Layer is far superior in it's ability to deal with error situations. while the NIL functions require." to a greater extent, that the user decide what the course of action will be for normal and abnormal conditions and bow will be implemented when an error occurs.
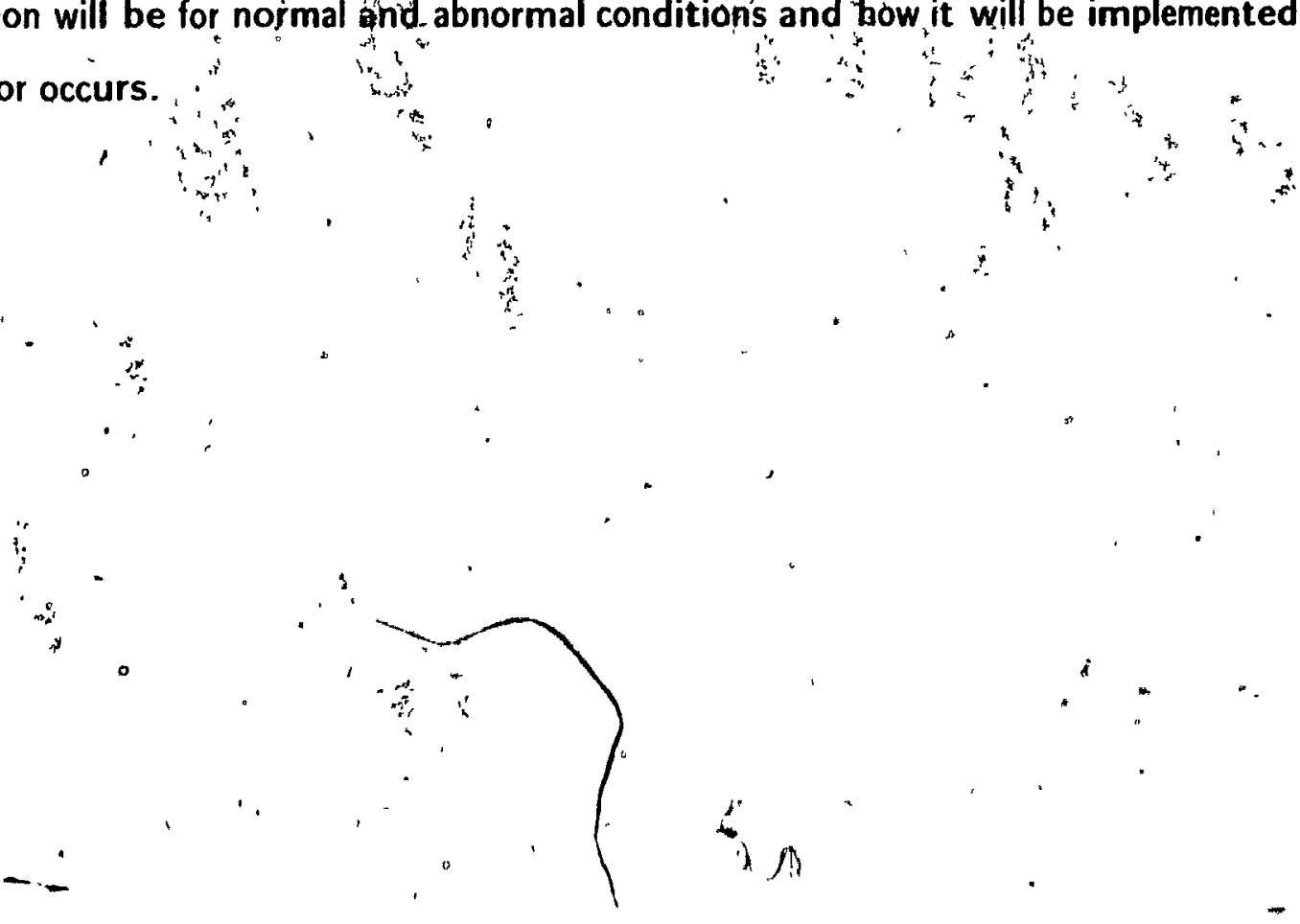

52 Performance in a Distributed System

larger data transfers: "this is therefore at the expense of the work-cell's communications needs. Although tuning affects the overall throughput rate. the same conditions are applied equally to both Session Layer and NIL programs since they are both based on TCP.

It is beyond the scoppe of this thesis to address the network performance below the level of the Session Layer and Network Interface Library. A performance evaluation of TCP. which includes the Transport. Network. Datä Link. and Physical Layers. in a network configuration similar to our own. composed of VAX and SUN hosts operating under UNIX 4.2BSD ảnd connected by an Ethèrnet is presented by Charison [Chanson èt àl. 84]. " In very general terms. the performance of the Transport Layer implementation depends on the following factors.

- The control štructure which: in part. deals with packet format. retransmission and timeout protócols, datá f́f fragmentation, and packet routing.

- The flow control mechanisms which are based on the available buffer space at the source and destination endpoints.

- The implementation of the protocol. In the case of 4.2BSD, the protocol is implemented in the UNIX kernel which, is the lowest level of the operating system and affords the greatest speed.

- The operatıng system overhead. This refers to the time consumed in process swapping. paging, system calls, and any other aspects pertàining to marshalling the environment of the processes.

\subsubsection{Network Contention}

Ethernet employs a carrier sense protocol for accessing the network. If a host has data to transport, it first interrogates the network for the presence of a carrier. that 
is. acket transmission present on the netwoik; If the channel is busy. it waits a fandom amount of time and then retries: otherwise. the data is transmitted. Network contention arises through the concurrent action of more than one host attempting to transmit a packet on the network. Ethernet does not employ a priority or queuing mechanism to felve contention. If a collision occurs. contention is resolved by having the hosts wait andom amount of time before retrying.

The performance of network protocols in the presence of contention is always decreased. Network contention can be partly evaluated by examining the collision rate of pàckets on the network Thıs rate is related to the number of packet retransmissions and "thus affects the performance. To some extent, network balancîng cân minimize contêntion by optimızing packet sizes and timeouts.

In our environment. the àverage collision rate is very low, approximately 100 parts per million. and is not considered to contribute any significant effects.

\subsubsection{Local versus Remote Communication}

If interprocess message passing is confined to a single host. the physical network is never accessed and contention can not arise. Because remote message passing requires network access. it is somewhat slower. Since in our environment network contention is very low. the effects of local versus remote communication are négligible.

\subsubsection{Processor Load}

Processor load. or C.PU load. plays a major role in network performance. TCP was designed to provide robust communication over lossy. long haul networks and therefore has extensive packetizạtion and error detection and correction features. This requires a tremendous amount of CPU resources: so much so that some argue that TCP is too pawerful for LAN applications. An alternative, less, robust protocol has been proposed by Chanson [Chanson et al. 85]. 


\subsubsection{Message Length}

$53^{*}$ Performance of the Implementation

The length of messages is certainly a contributing factor to network and system pêrformance. Messages designated to be transported are first stored in a TCP transmit buffer which is normally 2048 bytes in length. then packetized, usually in fragments of 128 bytes for TCP's stream protocol. and finally put on the network. The number of packets and ultimately the CPU load are proportional to the message length.

\subsubsection{Progràm Overhead}

Program overhead which entails calling routines to receive, parse. and send messages contributes to the end to end bandwidth. Efficient architectures and programming. both in implementing the network interface functions and on the part of the user who integrates these functions into their applications. is important.

\subsection{Performance of the Implementation}

The Session Layer performance was evaluated by comparing the times required to cycle a message, using both the Session Layer and NIL services. to and from a remote process.

The routines required to effect data transfers using the low level TCP functions amount to the send.msg and recvmsg functions of NIL. Therefore. from the perspective of this performance evaluation. the transmission times of both NIL and the TCP implementation can be considered to be identical. Henceforth the figures presented for the NIL implementation apply equally well to both NIL and TCP implementations

The time required to effect a message transfer depends on many factors. including the length of the message. the system loads. and the network balancing parameters which affect the performance of the Transport Layer. 


\section{( Our objective was, to evaluate the Session Layer relative to the Network Interface}

Library under the conditions of our present TCP installation. The actual times required to transport messages over the network are only relevant to our network configuration and tuning: relative comparisons are therefore made. NIL was chosen as the basis for performance comparison because it is the fastest TCP based mechanism available for transporting messages. We were not concerned with measuring the effects of factors specific to the TCP protocol. Thus, without loss of generality. we assumed that the time required to effect message transfers could be represented as an overhead tıme, due to TCP and the network interface functions. plus an increment which can be characterized as a function of message - length. Further, we congjer that both these parameters could themselves be dependent on the system load level and the type of host computer: VAX-11/750 (VAX). MicroVAX II (mVAX). or SUN System 2 (SUN). In evaluating the session layer, it was assumed that . data generated under almost identıcal conditions would not have been subjected to significant variations in overhead time; any error introduced by this simplification could be

$\because \quad$ absorbed into the overall errò, of the model without serrous consequence. In other words.

- the small fluctuations in system load encountered while performing the experiments were " not considered to be critical and therefore were not accounted for by the model.

In evaluating the Session Layer, we wanted the results to reflect typical conditions encountered in the laboratory. Since the packet collision rate is essentially zero. averaging. 100 parts per million, the effect of varying the network load was not deemed necessary. Thus measurements of load will be based strictly on. $C P U$ load, which for the purposes of this study. is defined as the number of jobs in the operatıng systems run queue averaged over the last one minute period.

\subsection{Experimental Design}

.. Programs which measure the time to cycle messages between two hosts using both the Session Layer and the NIL services were developed. A block diagram of the experimental setup is presented in Figure 5.1. 

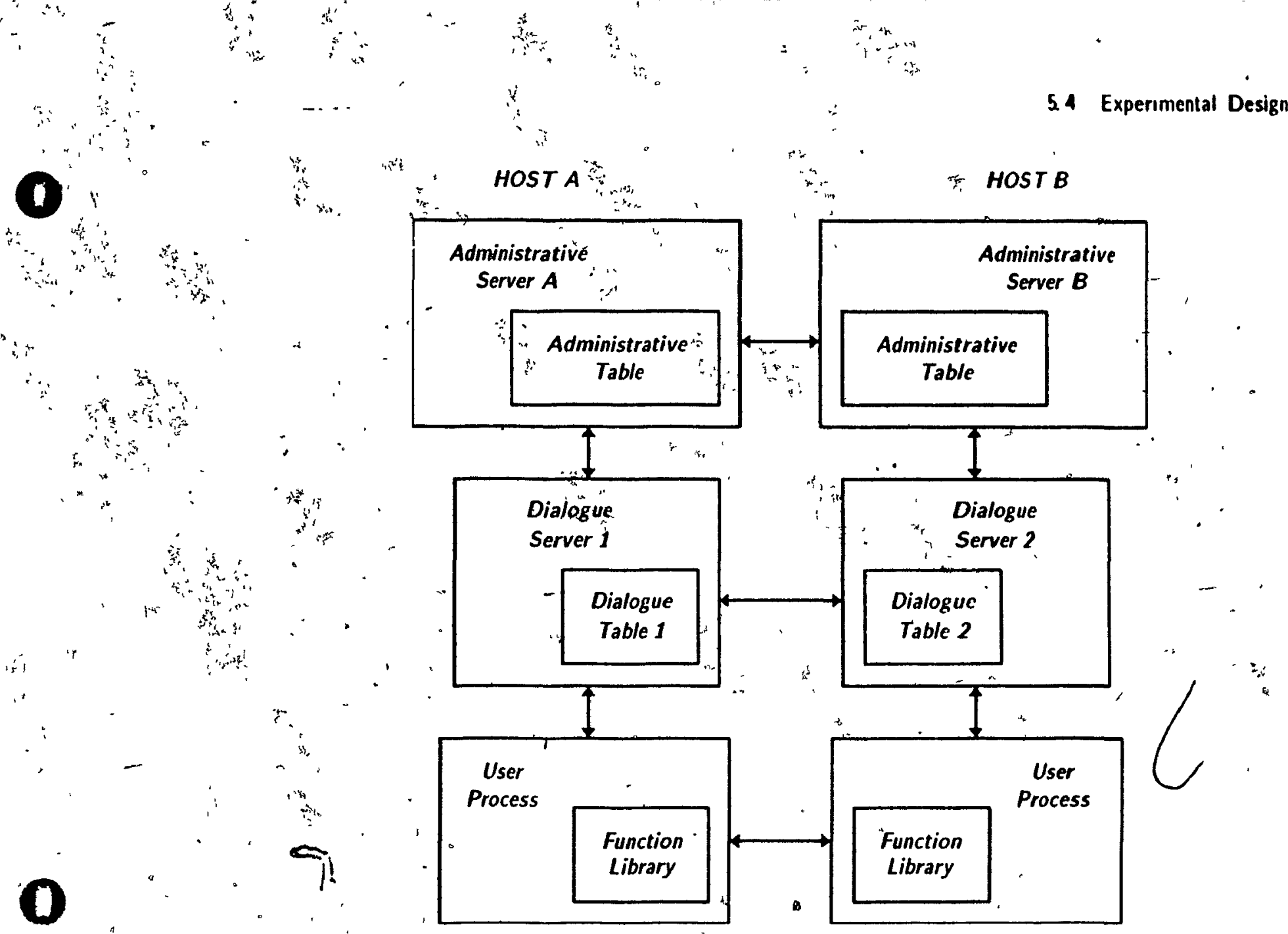

Figure 5.1 Configuration implementing both Session Layer and NIL links

Using the Session Layer facility. a message was transmitted from the local endpoint to the remote endpoint, and then returned. The elapsed time from the initial dispatch_msg function call to the return of the final read_msg call was measured. The message circulation was then immediately duplicated, this tıme over the direct socket connection. using the NIL send.msg and recv.msg functions. The tıme of the message transfer was again determined.

This procedure was performed in triplicate for messages of lengths ranging from 1 to 2048 characters, with an emphasis on lengths at the lower extreme of the range. An upper limit of 2048 characters was chosen because it corresponds to the transmit and receive message buffer sizes allocated to TCP when the system was configured.

To examine the influence of system load and computer host types. two sep- 



\subsubsection{Nariation of System Load}

It was assumed that the transmission time could be described by a relation involving the message ength, the system load level and the communication implementation - Ses'sion Layer or NIL. Other factors which could influence transmission time were assümed to remain constant. As will be more apparent latert. the consequence of an error in this assumption is that our estimbtion of the dependence of transmission time on system load may be in error. However. since our objective is to obtain delative comparison of the Session Layer to NIL. this donsequence should not be critical to aur analysis. Further. it was assumed that the colariance model which is described by equation 5.1 could be applied.
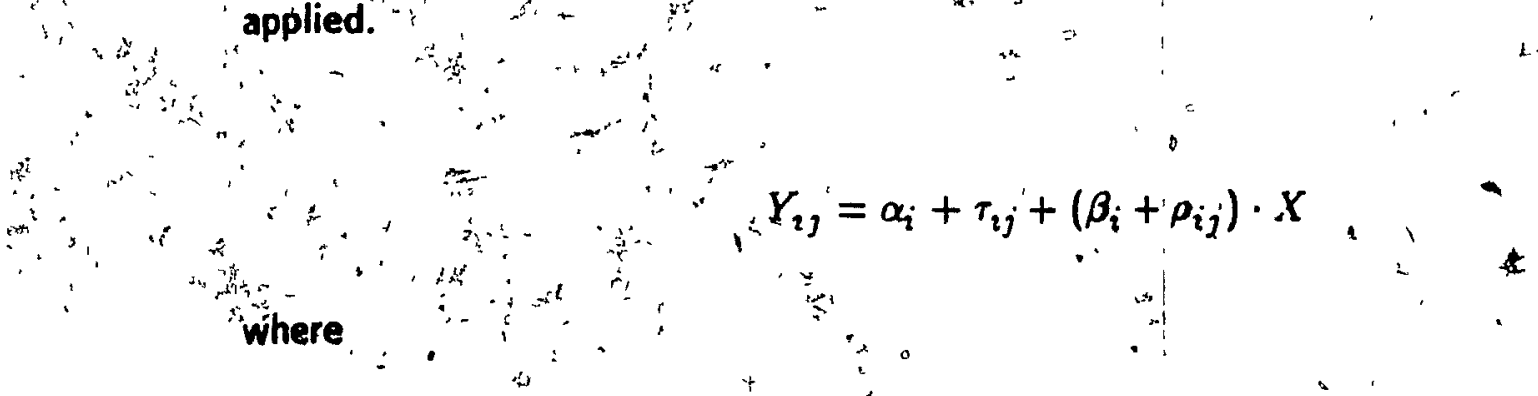

; $i$ deriotes the consistent system load on all machines: low. medium, or high. $i=0.1$. , 2:<smiles>[CH-]CC</smiles>

- $\quad j$ denotes the communiçation implementation: Session Layer or NIL. $\mathbf{j}=0,1$ :

$\checkmark$ medasures the time to circulate a message under load condition i using communication implementation $j$ :

; $X$ represents the message length:

$\alpha_{i}$ represents the component of the time. which is independent of the message length. required to circulate a message using NIL. under load condition $i$ :

$\beta_{i}$ measures the dependence on message length of the time to circulate a message using NIL under load condition i: 


The estimated parameters of equation 5.2 are tabulated in Figure 5.3 The additional time to circulate messages using the Session Layer rather than NIL was described by an overhead time which is independent of the message length, and a time which is directly proportional to the message length. The overhead. which is represented by $\tau$, was determined to increase with increasing system load. At low system load, the overhead - was estimated to be 1.62 seconds. while at high load, the overhead was estimated to be 1.93 seconds. The proportionality constant characterizıng the effect of message length on the Session Layer's performance, represented by $\rho$. was also determined to increase with system load. At low load. the proportionality constant was estimated to be 0.0022 $\mathrm{sec} /$ char. indicatıng that the additional tıme required by the Sessıon Layer to -transmit messages increases by 0.28 seconds for each increase of message length by 128 characters. At high system load. the additional transmissıon time required by the Session Layer to transmit a message was estımated to increase by 1.05 seconds for each 128 . character. increase in message length.

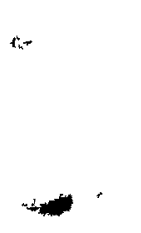

\begin{tabular}{|c|c|c|c|c|}
\hline $\begin{array}{l}\text { CPU } \\
\text { Load }\end{array}$ & $\begin{array}{c}\alpha \\
\sec \end{array}$ & $\begin{array}{c}\tau \\
\sec \end{array}$ & $\begin{array}{c}\beta \\
\mathrm{sec} / \text { char }\end{array}$ & $\stackrel{\rho}{\sec / \text { char }}$ \\
\hline low & 0.01 & 1.62 & 0.0021 & 0.0022 \\
\hline medium & 0.02 & 1.75 & 0.0049 & 0.0052 \\
\hline high & 0.04 & 1.93 & 0.0067 & 0.0082 \\
\hline
\end{tabular}

Figure 5.3 Parameter estimates of equation 5.2

In Figure 5.4, estimates of message transmission times based on the derived regression equation are presented for each system load level, for messages of lengths 2. 4. 8. 16, 32. 64. 128. 256: 512. 1024, and 2048 characters.

\section{- 5.5.2 Variation Between Host Computers}

The analysis of data generated by the second study. which considered varia-" tions in host computer types, maintained at mediüm system load, parallelled the analysis 
55 Analysis and Results

\begin{tabular}{|c|c|c|c|c|c|c|c|c|}
\hline \multirow[t]{2}{*}{$\because$} & & \multirow{2}{*}{$\frac{\text { Load }}{\text { Mode }}$} & \multicolumn{2}{|c|}{ Low } & \multicolumn{2}{|c|}{ Medium } & \multicolumn{2}{|c|}{ High } \\
\hline & & & NIL & SL & NIL & SL & NIL & SL, \\
\hline & & 2 & 0.02 & 1.65 & 0.03 & 1.79 & 0.05 & 2.00 \\
\hline & $\therefore$ & 4 & 0.03 & 1.66 & 0.04 & -1.81 & 0.07 & 2.03 \\
\hline & & & 0.04 & 1.67 & 0.06 & 1.85 & 0.09 & 2.09 \\
\hline & , & $16^{\circ}$ & 0.05 & 1.71 & 0.10 & 1.93 & 0.15 & 2.21 \\
\hline & & 32 & -0.09 & 1.78 & 0.17 & 2.09 & 0.26 & 2.45 \\
\hline$=$ & & 64 & 0.15 & 1.92 & 0.33 & 2.42 & 0.47 & 2.92 \\
\hline$\ldots$ & & 128 & 0.29 & 2.19 & 0.64 & 3.06 & 0.90 & 3.88 \\
\hline & $\therefore$ & 256 & 0.56 & 2.75 & 1.26 & 4.36 & 1.76 & 5.79 \\
\hline & & 512 & 1.10 & 3.86 & 2.51 & 6.94 & 3.49 & 9.60 \\
\hline & & & -2.19 & 6.08 & 4.99 & 12.11 & -6.94 & 17.23 \\
\hline$i$ & & 2048 & 4.36 & 10.53 & 9.97 & 22.46 & 13.84 & 32.49 \\
\hline
\end{tabular}

Figure $5.4^{\text {ob }}$ "Estimates of message transmission times (seconds).

described above. It was assumed that the transmission time could be described by a relation. involving the mesśage length. the computer types and the communication implementation Session Layer or NIL. Other factors which could influence transmission time were ąssumed to remąin constant. Since our objective was to obtain a relative comparison of the Session Layer to NIL, this assumption was not judged to be critical to our analysis.. It was also assumed, that the covariance model defined by equation 5.1 . with $i=0.1 .2 \ldots .5$ denoting the computer type. could be applied.

- The four variations of the model described by equation 5.1 were again considered. Each model was fitted to the data using the method of, least squares and the coefficient of correlation computed. The most appropriate model was selected. based on the coefficient of correlation. to again be the linear relation represented by equation 5.2 .

Simplification of the linear model was considered: the reduction of the model to one' which would assume that effects of the Session Layer were independent of computer " 
type was examined. F statistics were computed to evaluate the significance of the variations of the parameters $\tau$ and $\rho$ between various pairs of hosts. Both $F$ statistics were determıned to be highly significant. implying that the data demonstrated a difference according to host computer type of the effect of the Session Layer on both $\tau$, the overhead comporient of the message circulation time. and $\rho$. the time component which varies with message length. The model was therefore not reduced.

\begin{tabular}{|c|c|c|c|c|}
\hline \multirow{2}{*}{ Host } & $\alpha$ & $\tau$ & $\beta$ & $\rho$ \\
\cline { 2 - 5 } & sec & sec & sec/char & sec/char \\
\hline VAX/mVAX & 0.02 & 1.52 & 0.0017 & 0.0046 \\
VAX/VAX & 0.01 & 1.47 & 0.0024 & 0.0043 \\
SUN/VAX & 0.02 & 1.56 & 0.0018 & 0.0024 \\
mVAX/SUN & 0.01 & 1.42 & 0.0010 & 0.0016 \\
SUN/SUN & 0.01 & 1.39 & 0.0011 & 0.0020 \\
mVAX/mVAX & 0.01 & 1.44 & 0.0009 & 0.0014 \\
\hline
\end{tabular}

Figure 5.5. Parameter estimates for transmission times

The estimated parameters of equation 5.2 are tabulated in Figure 5.5. The overhead. which is described by $\tau$, was determined to range from 1.39 seconds for the SUN/SUN configuration to 1.56 seconds for the SUN/VAX configuration. a variation of 0.17 seconds. The proportionality constant characterizing the effect of message length on the Session Layer performance, described by $\rho$, was also determined to vary according to host configuration. The proportionality 'constant was estimated to 'range from $0.0014 \mathrm{sec} / \mathrm{char}^{\circ}$ for the $\mathrm{mVAX} / \mathrm{mVAX}$ to $0.0046 \mathrm{sec} / \mathrm{ch}$ ar for the VAX/mVAX configuration. In other words. the difference between transmission times of the Session Layer and NIL increased from the * overhead time by an amount ranging from 0.18 to 0.59 seconids for each additional 128 characters.

In Figures 5.6, and 5.7, estimates of message transmission times for NIL and Session Layer implementations are presented for each host computer type. for messages of lengths 2. 4. 8. 16, 32. 64. 128, 256. 512، 1024. 2048 characters. 


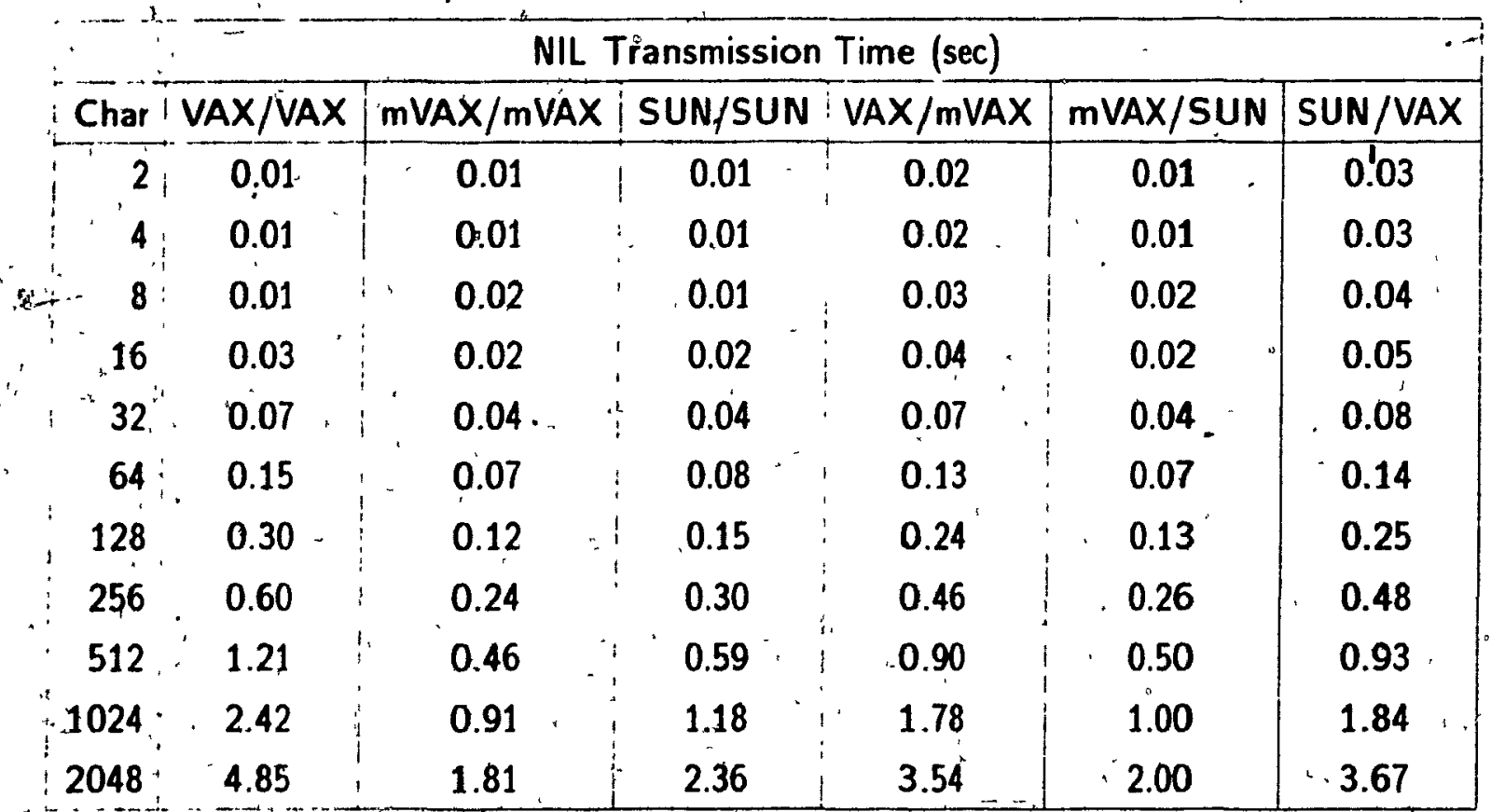

Figure 5.6 Estimates of NIL message transmission times

\begin{tabular}{|c|c|c|c|c|c|c|}
\hline \multicolumn{2}{|c|}{$i+1$} & \multicolumn{5}{|c|}{ Session Layer Transmission Time (sec) } \\
\hline Char & $\operatorname{VAX} / \mathrm{VAX}$ & $\operatorname{mVAX} / \mathrm{mVAX}$ & SUN/SUN & $\mathrm{VAX} / \mathrm{mVAX}$ & mVAX/SUN & SUN/VAX \\
\hline 2 & 1.48 & 1.46 & 1.40 & 1.55 & 1.43 & $1.59^{\circ}$ \\
\hline 4 & 1.49 & 1.46 & 1.40 & 1.56 & 1.43 & 1.60 \\
\hline 8 & 1.52 & 1.47 & 1.42 & 1.58 & 1.44 & . 1.61 \\
\hline 16 & $1: 57$ & $1.49^{n}$ & 1.44 & 1.64 & 1.46 & 1.65 \\
\hline $32 i$ & 1.68 & 1.53 & 1.49 & 1.74 & $1.50^{\circ}$ & 1.71 \\
\hline 64 & 1.89 & $\therefore 1.60$ & 1.59 & 1.94 & 1.59 & 1.85 \\
\hline 128 & 2.32 & 1.74 & 1.79 & 2.34 & 1.75 & 2.12 \\
\hline 256 & 3.17 & 2.03 & 2.19 & 3.15 & 2.07 & 2.66 \\
\hline 512 & 4.88 & 2.61 & $\because 2.98$ & 4.77 & 2.72 & 3.73 \\
\hline 1024 & 8.29 & $\quad 3.77$ & 4.58 & 8.00 & 4.01 & .5 .89 \\
\hline 2048 & 15,11 & 6.08 & 7.76 & -14.46. & 6.61 & 10.20 \\
\hline
\end{tabular}

Figurẹ 5.7 Estimateș of Session Layer message tranșmission times

\subsection{Discussion}


on the system load, the host computer type and the message length. Further, the analysis suggested that the relation between the message circulation time and the message length was linear.

- The difference in performance between the Session Layer and direct TCP. as measured by. NIL, was expressed as the sum of an overhead time which is independent of the message length. and an increment which is directly proportional to the message length. as was defined by equation 5.2. The overhead of the Session Layer was estimated to range from 1.39 to 1.93 seconds. depending on the system load and host configuration. The increment was observed to vary from as low as 0.18 to as high as 1.05 seconds per 128 character block transmitted in a message. That is. in addition to the overhead which varied between 1.59 and 1.93 seconds. each block of 128 characters transmitted will add from 0.18 to 1.05 seconds to the transmission time.

The variations in transmission times between host configurations did not imply that any particular host type will consistently enhance or impede the Session Layer's performance. The estimes of Session Layer overhead and increment times for each host computer combination indicated that the Session Layer transmission time is highest when a VAX host is involved. However, it was observed that the VAX configurations also exhibited the highest NIL transmission times. Thus. although data transmission is apparently slowest when a VAX host is involved. the relative performance of Session Layer and NIL based application programs is not diminished.

The times required to circulate messages between two processes using the Session Layer and NIL services are tabulated in Figure 5.4 as functions of message length for low. medium and high system loads. for the VAX/VAX host configuration. In Figures 5.6 and 5.7, similar results are presented for host configurations of VAX/VAX, mVAX/mVAX. SUN/SUN. VAX/mVAX, mVAX/SUN. SUN/VAX. based on medium system loads. Three observations are immediate from Figures 5.4. 5.6. and 5.7: the Session Layer results are consistently higher than the NIL times. the transmission times increase as the message length increases, and the transmission tımes increase as the system load increases. 
In our applications, the majority of messages passed are expected to be short. not exceeding 32 characters. In order to better understand the performance characteristics. of the Session Layer, a bound on the difference in' performance of the Session Layer and NIL for messages of this length was estimated. The difference in message transmission times between the Session Layer and NIL for 32 character messages were determined from Figures 5.4. 5.6, and 5.7. For a VAX/VAX host configuration. Figures 5.6 and 5.7 estimated the additional time required by the Session Layer to be 1.61 seconds, while Figure 5.4 estimated the time to be as high as 2.19 seconds in the presence of a high load. a variance of $36 \%$. This variance applies directly to the pair of machines. VAX/VAX. on which the measurements were made. Although the effect of load on the Session Layer's performance cannot be assumed to be constant over all machines. it would be reasonable to expect that comparable loads would produce similar effects. Applying the $36 \%$ variance to the estimated difference in Session Layer and NIL transmission times for other host configurations, it was predicted that the additional time expended by the Session Layer should not exceed 2.3 seconds at high system load for any host combination. Thus, the additional time required for most messages transmitted via the Session Layer is not expected to exceed 2.3 seconds. regardless of system load and host computer configuration.

"It is finteresting to notice from Figures 5.4. 5.6. and 5.7 that the effects of
message Idngth would be more evident in NIL than in Session Layer applications. As the message length increased from 2 characters to 2048 characters. the time to circulate a message using the Session Layer did not increase by more than 17 fold for any level of system load or any host computer combination; while the time to circulate a message using NIL functions increased by more than 100 fold for all load levels and host configurations. In Figure 5.8. the ratio of the times to transmit a message using NIL to those for the Session Layer, determined from Figures 5.6 and 5.7. are tabulated for each host computer configuration, for messages of lengths 2. 4. 8. 16. 32.64.128. 256. 512, 1024 and 2048 characters. 


\begin{tabular}{|r|c|c|c|c|c|c|c|}
\hline \multicolumn{7}{|c|}{ NIL / Session Layer Transmission, Times } \\
\hline Char & VAX/VAX & mVAX/mVAX & SUN/SUN & VAX/mVAX & mVAX/SUN & SUN/VAX \\
\hline 2 & .007 & .007 & .007 & .013 & .007 & .019 \\
4 & .007 & 007 & .007 & .013 & .007 & .019 \\
8 & .007 & .014 & .007 & .019 & .014 & .025 \\
16 & .019 & .013 & .014 & .024 & .014 & .030 \\
32 & .042 & .026 & .027 & .040 & .027 & .047 \\
64 & .079 & .044 & .050 & .067 & .044 & .076 \\
128 & .129 & .069 & .084 & .102 & .074 & .118 \\
256 & .189 & .119 & .137 & .146 & .126 & .180 \\
512 & .248 & .176 & .198 & .189 & .184 & .249 \\
1024 &. .292 & .241 & .258 & .223 & .249 & .312 \\
2048 & .321 & .298 & .304 & .245 & .303 & .360 \\
\hline
\end{tabular}

Figure 5.8 Ratio of NIL to Session Layer performances

The discrepancy between the effect of increased message length on the Session Layer and NIL performances is clearly indicated by the consistent increase in the performance ratio with increasing message ilength. demonstrated by all host configurations. This apparent discrepancy can be attributed to the fact that the overhead of the Session Layer, $\alpha+\tau$ is much/farger than the overhead of NIL; $a$.

The performance ratio. tabulated in Figure 5.8, is illustrated graphically in Figure 5.9 for the VAX/VAX host configuration. A logarithmic scale is utilized for the message length axis to highlight the ratio at short message lengths. The ratio intially remains low, reflècting the relatively high overhead of the Session Layer over NIL. As the message length increases. the performance ratio increases. but not in such a way that an optimal message length for either the Session Layer or NIL can not be identified. The data revealed that as the message length approaches the size of the TCP transmit and receive buffer. 2048 bytes, the message transmission speed was almoșt solely dependent on the systems' efficiency in managing the buffers. 

depends on communication mediated by servers as opposed to NIL's which specifies direct links between end processes. The Session Layer was shown to exhibit a relatıvely sıgnificant message transmission time which is primarily due to a large overhead. that is, the time necessary to transmit a message of length zero. It was shown that the time to trănsmit messages via the Session Layer is directly proportional to CPU load and message length The analysis also suggested a dependence on host configuration. but no individual host could be identified as either enhancing or impeding the performance. The data indicated that high Session Layer transmission times were accompanied by high NIL transmission 'times' indicating that data transmission in general, and not the Session Layer's relative "performance, could be impeded by any particular host. This phenomenon could be studied in the future.

The primary objective of the Session. layer is to facilitate access to the network and standardize the method of doing so. Although performance is not a primary concern. we must consider whether or not the observed performance is acceptable for our applications.

In our opinion. the performance of the Session Layer and NIL. either separately or together is satisfactory. Under worst case conditions. which occur very rarely, we expect that the Session Layer's end-to-end-message transmission time will not exceed 2.3 seconds for a 32 character message. The present bottlenecks in our work-cell applications are image processing and to a lesser extent, robot tasks which are usually of an order of magnitude and two or three times that of the Session Layer respectively Even if their speeds approached or were faster than that of the Session Layer, because of our distributed design philosophy the worst that could happen would be a very short pause in the work-cell activities. 'In our applications, task loads are partitioned. in such a, way as not to require high speed transactions, as would be needed for servo control or some types of image processing. These tasks are consolidated on the same host to avord excessive network transactions. If a situation should ever arise that requires more efficient message passing. the user can always integrate NIL functions into their programs to provide better performance. 



[Alford and Belyeu 84] Alford. "C.. Belyeu. S.. "A Computer Control Structure for Coordination of Two Robot Arms." Proc. American Control Conf. 1984.

[Andrews 82] Andrews. G.. "The Distributed Programinging Language SR - Mechanisms. Design, and Implementation," Software Practice and Experience, Vol. 12 1982.

[Andrews and Schneider 83] Andrews. G.. Schneider. F.. "Concepts and Notations for Concurrent Programming." ACM Computing Surveys. Vol.' 15. no. 1. March 1983.

[Baird et al. 84] Baird. H., Wells, E.. Britton. D., "Coordination Software for Robotic Workcells." Proc. IEEE Int. Conf. on Robotics and Automation. 1984.

[Baker and Riccardi 85] Baker. T.. Riccardi. G., "Ada Tasking: From Semantics to Efficient Implementation," IEEE Software. March, 1985.

[Barthes and Zavidovique 81] Barthes. J.. Zavidovique. B., "How Much Intelligence should we expect from a Vision Processor in a Multi-processor Robot System ?." Proc. 1st Int. Conf. on Robot Vision and Sensory Controls. 1981.

[Bejczy and Lee 84] Bejczy. A.. Lee. S., "Generalized Bitateral Control of Robot Arms." Proc. American Control Conf., 1984.

[Birrell and Nelson 83] Birrell. A.. Nelson, B.. "Implementing Remote Procedure Calls." Proc. 9th ACM Symp. on Operating Systems Principles. 1983.

[Bonner and Shin 82] Bonner, S., Shin, K.: "A Comparative Study of Róbot Languages," IEEE Computer, December 1982.

[Bonin et al. 83] Bonin. J.. Elloy. J., Haurat. A.. Molinaro. P., Thomas. M.. "The Tools for Synchronizaton of the LMAC System for the Cooperation of Industrial Robots." Proc. Int. Conf. on Advanced Software for Robotics. 1983.

[Brinch-Hansen 75] Brinch-Hansen. P.. "The Programming Language Concurrent Pascal." IEEE Trans. on Software Eng.. June 1975.

[Bruno et al." 84] Bruno: G.. Demartini, C.; Valenzano. A.: "Communication and Programming Issues in Robotics Manufacturing Cells." Proc. IEEE Int. Conf. on Robotics and Automation, 1984.

[Carayannis 82] Carayannis. G.. "Controlling a PUMA 260 Robot from a VAX Computer," Technical Report TR-83-3. Computer Vision and Robotics Lab.. McGill University, April 1983. 
[Chanson et al. 84] Chanson. S.. Ravindran. K.. Atkins. S., "Performance Evaluation of the ARPANET fransmission Control Protocol in a Local Area Network Environment." Technical Report 85-6. Department of Computer Science. The University of British Columbia, Vancouver. B.C.. 1984.

[Chanson et al. 85] Chanson. S., Ravindran. K.. Atkins. S.. "LNTP - An Efficient Transport 'Protocol for Local Area Networks." Technical Report 85-4. Department of Computer Science. The University of British Columbia. Vancouver. B.C.. 1985.

[Chen et al. 83] Chen. J.. Loh. H., Chıang. K., Say. K. "A Software Package for Microrobot and Coordination between Robots with Voice Command." Proc IEEE Annual Conf. on Industrial Electronics (IECON). 1983.

[Clark 82] Clark. D.. "Modularity and Efficiency in Protocol Implementation." Technical Report. Computer Systems and Communication Group. MIT Laboratory for Computer Science. 1982

[Devarakonda et al. 85] Devarakonda. M.. McGrath. R.. Campbell. R., and Kubitz. W.. "Networkıng a Large Number of Workstations" Using UNIX United." Proceedings $\therefore$ of IEEE Workstations Conference. November 1985.

[Emmons and Chandler 83] Emmons. W.. Cliandler. A., "OSI Sessıon Layer: services and protocols." Proc. IEEE. Vol 71. no. 12. December 1983.

[Draper et al. 66] Draper:*N. Smith. H.. Applied Regression Analysis. John Wiley and Sons. Inc.. New York. N.Y.. 1966.

[Dwivedi 83] Dwivedi, S.. "Design of a Low Cost Remote Control Unit for a Robot.", Proc. IEEE Southeastcon. 1983.

[Elfes and Talukdar 83] Elfes. A.. Talukdär. S.. "A Distributed Control System for the" CMU Rover." Proc. 1983 IJCAI Conf.. 1983.

[Elgazzạr et al. 84] Elgazzar. S.. Green. D.. O'Hara. D.. "A Vision-based Robot System using a Multiprocessor Controller," NRCC no. 23485. June 1984.

[Faro and Messina 83] Faro. A.. Messina. G.. "Robot Internetworking," IEEE Trans. on Industrial Electronics. November 1984.

[Faro and Messina 84] Faro. A.. Messina. G., "Error Mahagement in Robot Environment." Proc. IEEE TENCON (Trends in Electronics) Conf., 1984.

[Faro et al. 85] - Faro. A.. Mirabella. O.. Vita. L.. "A Multimicrocomputer-based, Structure for Còmputer Networking." IEEE Micro. April 1985.

[Fathi et al. 83] Fathi. E.. Krieger. M.. "Multiple Microprocesessor Systems: What. Why. and When." IEEE.Micro. March. 1983. 
[Fisher and Weatherly 86] Fisher. D.. Weatherly. R.: "Issues in the Design of a Distributed Operatıng System for Ada." IEEE Computer. May 1986.

[Garetti et al. 82] Garettı. P.. Laface. P... Rivoîra. S.. "MODUŚK: A Modular. Dístributed Operating System Kernel for Real-Tıme Process Control," Microprocessing and Microprogramming. Vol 9. 1982.

[Gauthier et at. 85] Gauthier. D.. Carayannıs; G.. Freedman. P., Malowany. A.. "A Session Layer Design for the CVaRL Local Area Network:" Technical Report TR-857R. Computer Visıon and Robotics Lab.. McGill University. February 1985.

[Gentleman 83] Gentleman. W.. "Using the HARMONY Operating System." NRCC no. 23030. December 1983.

[Goldwasser 84] Goldwasser. 'S.. "Computer' Architecture for Grasping." Proc. IEEE - Int. Conf. on Robotics and Automation. 1984.

[Goldwasser and Bajcsy 83] Goldwasser. S.: Bajcsy, R., "A Distributed Active Sensory "Processor 'System." Proc. 3rd Scandinavian Conf. on Image Analysis. 1983.

[Gonzalez and Safabakhsh 82] Gonzalez. R.. Safabakhsh. R.. "Computer Vision Techniques for Industrial Applications and Robot Control," IEEE Computer. Vol. 15, nơ 12. December 1982.

[Green 83] Green. D., "CHORUS: A Multiprocessor Architecture for Real-Time Control - Applications:" NRCC no. 23031. December 1983.

[Gruver et al. 83] Gruver. W.. Soroka. B., Craig. J . Turner. T.. "Evaluation of Commercially Available Robot Programming Languages." Proc 13th Int. Symp. on Industrial Robots. 1983.

' [Hanlon and Weston 82] Hanlon. P. Weston. R.. "Use of Local Area Networks within Mạnufacturıng Systems." Microprocessors and Microsystems. October 1982.

[Harmon 83] Harmón. S.. "Co-ordination between Control and Knowledge-based Systems for Autonomous Vehicle Guidance." Proc. IEEE Trends and Applications Conf.. 1983.

[Harmon and Gage 80]. Harmon, S.. Gage. D.. "Protocols for Robot Communications. Transport and Content Layers." Proc. IEEE Int. Conf on Cybernetics and Society. 1980.

[Harmon et al. 84] Harmon. S.. Gage, D.. Aviles. W.. Bianchini. G.. "Coordination of Intelligent Subsystems in Complex Robots." Proc. IEEE 1st Conf. on Artificial Intèlligence Applications. 1984.

[Hayward and Paui 83] Haywärd. V.. Paul R. P.. "Robot Manipulator Control Úsing 
the 'C' language under UNIX." Proc. IEEE Workshop on Languages for Automation, November' 1983.

[Hoberecht 80] Hoberecht.V.. "SNA Function Management." IEEE Trans. Communications. Vol. 28. no. 4. April 1980.

[Holland 83] Holland. J.. "Local Area Networks: A Critical Part of Factory Automation." Proc. Numerical Control Society's Technical Conf. 1983.

[Holmgren 85] Holmgren. S.. "The Untapped Potential of Remote Procedure Calls." UNIX Review. May 1985

[ISO 7498] "OSI Basic Reference Model." ISO TC97/SC16. N.7498. 1982. ISO Central Secretatiat. 1 rue de Varembe. 1211 Geneva 20. Switzerland.

[ISO 8072] "Information Processing Systems - OSI - Transport Protocol Specification." N.8073. 1985. ISO Central Secretatiat. 1' rue de Varembe. 1211 Geneva 20. Switzerland.

[ISO 8073] "Information Processing Systems - OSI - Transport Service Definition." N.8073. 1985. ISO Central Seçretatiat, 1 rue de Varembe. 1211 Geneva 20. Switzerland.

[IŚO 8326] "Information Processing Systems - OSI - Session Service Definition." N.8326. 1984. ISO Central Secretatiat, 1 rue de Varembe, 1211 Geneva 20. Switzerland.

[ISO 8327] "Information Processing Systems - OSI - Basic Connection Oriented Șession Protocol Specification." N.8327, 1984. ISO Central Secretatiat. 1 rue de Varembe. 1211 Geneva 20. Switzerland.

[ISO 8473] "Data Comunicatións Protocol for Providing Cónnectionless M̀òe Internet Network Service." N.8473. ISO Cèntral Secrétatiat, 1 rue de Varembe. 1211 Geneva 20. Switzerland.

[ISO 8571] “Information Processing Systems - OSI - File Transfer. Access, and Management." N.8571/1/2/3/4. ISO Central Secretatiat. 1 rue de Varembé. 1211 Geneva 20. Switzerland.

[ISO 8649] "Information Processing Systems - OSI - Common Application Service elements Servicé Definition." N.8649. iso Central Secretatiat, 1 'rue de Varémbe. 1211 Geneva 20. Switzerland.

[1SO 8802] "IEEE 802.1/2/3/4/5 LAAN Standard Documents." N.8802. ISO Central Secretatiat. 1 rue de Varembe. 1211 Geneva 20. Switzerland. 
[Iversen 85] Iversen. W.. "Factory Automation Advances Apace." ElectronicsWeek. Feb 18. 1985.

: [Jarvis 84] Jarvis, J.. "Robotics," IEEE Computer. Vol. 17. nio. 10; October 1984:

[Joseph 74] .Joseph. E., "Distributed Function Computer Syšstems Innovativè Trends." Digest of Papers. IEEE COMPCON 1974. Spring. 1974.

[Kaminski 86] Kaminski. M.. "Protocols for Communicating in the Fàctory." IEEE Spectrum. April 1986.

[Kärkkäinen 83] Kärkkäinen. P.. "A Sensor Information Preprocessıng System for Manipulators based on Distributed Microcomputers." Proc.- Conf. on Advanced Software in Robotics, 1983:

[Kärkkäinen and Manninen 83] Kärkkäinen. P.. Manninen. M.., "A Hierarchical Dístributed Information Processing System for 'Forest Manipulation." Proc. IFAC Real Time Digital Control Applications Conf.. 1983.

[Leopold 84] Leopold, G.. "Factory Nets Follow ą MAP." ElectronıcsWeek. Dec. 17. 1984.

[Lloyd 85] Lloyd. J.. "Implementation of a Robot Development Environment." M. Eng Thesis. McGill University, Montreal, Quebec, 1985.

[Michaud et al. 85] Michaud. C.. Malowany. A., Levine. M "Multi-robot Assembly of Electronic Circuits." Proc. Graphics Interface 85. 1985.

[Ma and K'rishnamurti 84] Ma. Y.-W.. Krishnamurti. R., "REPLICA - A Reconfigurable Partionable Highly Parallet Computer Architecture for Active Multi-sensory Perception of 3-Dimensional Objects." Proc. IEEE Int. Conf. on Robots and Automátion. 1984.

[MacWilliams et al. 84] MacWilliams. P.. Wolochow P.. Zasloff R., "Microcontroller. Serial Bus Yields Distributed Multispéed Control." Electronics. Feb.; 9. 1984.

[Maimon 85] Maimon. O.. "A Multi-robot Control Experimental System with Random Parts Arrival." Proc. JEEE Int. Conf on Robots and Automation. 1985

[Maimon and Nof 83] Maimon. O.. Nof, S.. "Activity Controller for a Multiple Robot Assembly Cell.". Proc. Winter Annual Meeting of the ASME. 1983.

[Makhlin 82] Makhlin. A., "Vision Controlled Assembly by a Multiple Manipulator Robot." Proc. 2nd Int. Conf on Robot Vision and Sensory Controls, 1982.

[Mao and Yeh 80] Mao. T.. Yeh. R.. "Communication Port: A Language concept for Concurrent Programming." IEEE Transactions on Software Engineering. March. 1980.

-[MAP 85] "Manufacturing Automation Protocol. Version 2:1." Mànufacturing Engineering and Development. General Motors Technical Center. Manufacturing Building. 
A/MD-39, 30300 Mound Road. Warren. MI. 48090-9040. 1985.

[McQuillan and Walden 77] - McQuillan. J.. Walden. D.. "The ARPA Network Design Decisions.". Computer Networks. Vol.1. August 1977.

[Metcalfe and Boggs 76] Metcalfe.R., Boggs.:- D.. "ETHERŅĖT: Dịstributed Packet Switching for Local Computer Networks." Comm. ACM. Vol.19. July'1976...

[Michaud 85] Michaud: C., "MROUTINES.C: Using the Microbo Robot with Style." Technical Report TR-85-3. Computer Vision and Robotics Lab.. McGill University. Januagy 1985.

[Milne 83] Milne. B.. "Board Testing: The Future is Software. Networking," Electronic Design. Nov. 24. 1983

[Nagel 83] Nagel. R.. "Robots: not yet smart enough.". IEEE Spectrüm. Vol. 20. na. 5. May 1983.

[Neumann 83] Neumann. J.." "OSI Transport and Session Layer Services and Protocols." Proc. IEEE Infocom Conf., 1983.

[Pearson and Green 84] Pearson. J.. Green. D.. "Three Channel Infrared Wireless Eink." Proc. IEEE Sóutheastcon. "1984.

[Plessmann 83] Plessmann. K., "A Multi-microcomputer-based Robot Control System." : Proc. IFAC Real Time Digital Control Applications Conf., 1983.

[SAS 82] SAS Institute Inc., SAS User's Guide: Statistics. 1982 Edition. SÄS Institute. Inc. Cary. North Carolina. 1982.

[Searle 71] Searle. S.. Linear Models, John Wiley and Sons. Inc., New York. N. Y.. 1971,

[Shatz 84] Shatz. S.. "Communication Mechanisms for Programming Distributed Systems." IEEE Computer, June 1984.

[Shimano et al. 84] Shimano. B.. Geschke. C., Spalding. "C.. "VAL II: A' New Robot Control System for Automatic Manufacturing," Proc. IEEE Int. Conf. on Robotics and Automation. 1984.

[S hin and Epstein 85] Shin. K.. Epstein. M.. "Communication Primitives for a Distributed Multi-robot System." Proc. IEEE Int. Conf. on Robotics and Automation. 1985.

[Silverman 84] Silverman. J., "Communications in a Distributed Computer Testbed." IEEE Int. Conf. on Distributed Computing Systems, 1984.

[SRI 82] Transmission Control Procedure/Internet Protocol. Internet Workbook, Network Information Center. SRI International. March 1982. 
[Stankovic 82] Stankovic. J.. "Software Communication Mechánisms. Procedure Calls versus Messages." IEEE Computer. Vol. 15. no." 4. April 1982

[Stauffer 85] Stauffer, R.. "Three-robot Workcells Help Assemble Solid-state Ignition Modules." Robotics Today: February 1985.

[Storoshchuk et al. 83] Storoshchuk. O.. Szabados. B.. "Industrial Local Area Net. work." Proc. IEEE Int. Electrical and Electronics Conf.. 1983.

[Sun 85] - Sur Microsystems Inc. "Networking on the Sun Workstation - Inter-Process Comunication Primer." Part No. 800-1177-01. Release 2. May 1985.

[Tannenbaum 81] Tannenbaum. A . Computer Networks. Prentice-Hall. 1981.

[Taylor et al. 82] Taylor. R.. Summers. P.. Meyer. J., "AML: A Manufacturing Lan: guage." Int. Journal of Robotics. Research. Fall 1982.

[Tuthill 85] .Tuthill, B.: "IPC Facilities in 4.2BSD," Unix Review. April 1985.

[UCB 83], 4.2BSD System Manual. Computer Systems Research Group. Univerșity of California - Berkeley. July 1983. ACM Symp. on Operating Systems Principles. 1983.

[Unimation 86] Unımation Inc., "Robot Controller Under development." IEEE Computer. Vol. 19. no. 6. June, 1986.

[Voelcker 86] . Voelcker. J.. "Helping Computers Communicate." IEEE Spectrum. April' 1986.

[Volz et al. 84] Volz. R.. Mudge., T.. Gal, D.. "Using ADA as a Programming Lan guage for Robot-based Manufacturing Cells." IEEE Trans. on System. Mañ. and Cybernetics. November/December 1984.

[Wainwright and Moss 85] Wainwright, R., Moss', R:, "A Microcomputer -based Model Robot System with Pulse-width Modulation Control," IEEE Micro. February 1985

[Willis and Sanderson 84] Willis. J., Sanderson. A.. "RAPIDbus: Design of an Extensible Multiprocessor Strucțure." Technical Report no. 84-13 from The Robotics Institute of Carnegie-Mellon University. 1984.

[Wirth 77] Wirth. N., "Modula - A Language for Modular Multiprogramming." Software. Practice and Experience. January 1977.

[Xerox 85] Xerox Network Systems Architecture - General Information Manual, XNSG 068504. Xerox Cosporation, 1985.

[Zimmermann 80] Zimmermann. H., "OSI Reference Model - The ISO Model for Open Systems Interconnection." IEEE Trans. Comm.. April 1980. 